\title{
Methylation risk scores are associated with a collection of phenotypes within electronic health record systems
}

Mike Thompson ${ }^{\dagger 1}$, Brian L. Hill ${ }^{\dagger 1}$, Nadav Rakocz ${ }^{1}$, Jeffrey N. Chiang ${ }^{2}$, IPH $^{3}$, Sriram

Sankararaman $^{1,2,4}$, Ira Hofer ${ }^{5}$, Maxime Cannesson ${ }^{5}$, Noah Zaitlen ${ }^{6}$, and Eran Halperin ${ }^{1,2,3,4,5,7}$

mjthompson@ucla.edu,brian.l.hill@cs.ucla.edu, ehalperin@cs.ucla.edu*,

${ }^{*}$ Corresponding Author

${ }^{\dagger}$ Denotes equal contribution

${ }^{1}$ Department of Computer Science, University of California Los Angeles, Los Angeles, CA, USA

${ }^{2}$ Department of Computational Medicine, University of California Los Angeles, Los Angeles, CA, USA

${ }^{3}$ Institute of Precision Health, University of California Los Angeles, Los Angeles, CA, USA

${ }^{4}$ Department of Human Genetics, University of California Los Angeles, Los Angeles, CA, USA

${ }^{5}$ Department of Anesthesiology and Perioperative Medicine, University of California Los Angeles, Los Angeles, CA, USA

${ }^{6}$ Department of Neurology, University of California Los Angeles, Los Angeles, CA, USA

${ }^{7}$ OptumLabs, Minnetonka, MN, USA 


\section{Abstract}

2 Inference of clinical phenotypes is a fundamental task in precision medicine, and has therefore been heav3 ily investigated in recent years in the context of electronic health records (EHR) using a large arsenal of 4 machine learning techniques, as well as in the context of genetics using polygenic risk scores (PRS). In this work, we considered the epigenetic analog of PRS, methylation risk scores (MRS), a linear combination of methylation states. Since methylation states are influenced by both environmental and genetic factors, we hypothesized that MRS would complement PRS and EHR-based machine-learning methods, improving overall prediction accuracy. To evaluate this hypothesis, we performed the largest assessment of methylation risk scores in clinical datasets to be conducted to date. We measured methylation across a large cohort $(\mathrm{n}=831)$ of diverse samples in the UCLA Health biobank, for which both genetic and complete EHR data are available. We constructed MRS for 607 phenotypes spanning diagnoses, clinical lab tests, and medication prescriptions. When added to a baseline set of predictive features, MRS significantly improved the imputation of 139 outcomes, whereas the PRS improved only 22 (median improvement for methylation $10.74 \%, 141.52 \%$, and $15.46 \%$ in medications, labs and diagnosis codes, respectively, whereas genotypes only improved the labs at a median increase of $18.42 \%$ ). We added significant MRS to state-of-the-art EHR imputation methods that leverage the entire set of medical records, and found that including MRS as a medical feature in the algorithm significantly improves EHR imputation in $37 \%$ of lab tests examined (median $R^{2}$ increase $47.6 \%$ ). Finally, we replicated several MRS in multiple external studies of methylation (minimum p-value of $2.72 \times 10^{-7}$ ) and replicated 22 of 30 tested MRS internally in two separate cohorts of different ethnicity. In summary, our work provides a comprehensive evaluation of MRS in comparison to PRS and EHR imputation on the largest dataset consisting of methylation, genotype, and EHR data. Our publicly available results and weights show promise for methylation risk scores as clinical and scientific tools.

\section{Introduction}

Widespread adoption of electronic health record systems coupled with an increasing interest in hospital biobanking systems has spurred research efforts spanning machine-learning and genomics communities 1 7. These efforts have produced increasingly accurate imputation (current state) and prediction (future state) of patient phenotypes from medical records 8,9 and polygenic risk scores $[1,3,10,14$, and are already being investigated in translational contexts [15 18]. For example, recent work has shown that machine learning can leverage high-dimensional data to aid in the prediction of a multitude of clinical phenotypes including cardiac function and arrhythmia 19 21], post-operative complications [8, 9], sepsis [22, breast cancer [11, 23], and prostate cancer 24]. Nonetheless, a genetics-based predictor such as the polygenic risk score may be limited in predictive utility as it does not account for changes in disease risk - for example, due to age, or changes in environment - throughout one's lifespan [13].

In this work we examine the potential for epigenetic information to improve phenotype inference in combined biobank-EHR systems. As DNA methylation, henceforth referred to as simply "methylation", is affected by both genetics and environment - such as lifestyle choices, diet, exercise, and smoking status - it captures multi-factorial information about predispositions to clinical conditions 25 31. Moreover, methylation is readily available for use in existing biobanks that collect DNA samples, and recent advancements in methylation profiling technologies have enabled an abundance of large-scale studies of methylation and its role as a biomarker for a variety of phenotypes and health-related outcomes 25, 3137. It is therefore a natural candidate for an extension of PRS, and we hypothesized that methylation can be used to complement genetics as a clinical prediction tool. To that end, we have generated and evaluated methylation risk scores (MRS), which are linear combinations of CpG methylation states 25.

To comprehensively investigate the utility of MRS and characterize its properties, we conducted a study of 607 EHR-derived phenotypes spanning medications (e.g. vasopressers, glucocorticosteroids, fluoroquinolones), labs (e.g. creatinine, glucose, prothrombin time), and diagnoses (e.g. T2D, bacterial 
pneumonia, anemia) that were available for a sufficient number of patients in the cohort. The cohort contained 831 patients - to the best of our knowledge, the largest epigenetic biobank dataset to date (including genetics, methylation, and EHR) - from the UCLA Health ATLAS cohort across a wide range of ages (18-90), racial and ethnic groups, and overall health (including patients ascertained on kidney and heart disease, with matched controls), with corresponding genetic and EHR data. This provides the opportunity to study the potential contribution of methylation to larger biobanks and in multiple clinical contexts. We find that the MRS-based imputations were more informative compared to PRS in $84(92 \%)$ medications, 32 (94\%) labs, and $123(82 \%)$ diagnoses, more than doubling the imputation accuracy in over half of the outcomes considered. We also show that the MRS improves the imputation accuracy over PRS for cases in which the PRS is trained on very large external biobanks (roughly 3 orders of magnitude larger), as opposed to 831 samples that are available in this study. We observe that MRS improves over PRS learned from large biobanks in $40 \%$ of the tested phenotypes. Further, as our cohort was ethnically diverse, we performed replicability analyses within each racial and ethnic subset of our data. We broadly showed the replicability of the five best-imputed (by MRS) medications, labs, and diagnoses $-46 \%$ and $100 \%$ of which replicated in $(\mathrm{n}=118)$ non-white Hispanic-Latino- and $(\mathrm{n}=543)$ white non-Hispanic-Latino-identifying individuals respectively. Finally, we demonstrate the ability of MRS to transfer between methylation arrays and cohorts by conducting an association study of kidneyrelated MRS in an external diabetic nephropathy EWAS [38], where the minimum replication p-value was $2.72 \times 10^{-7}$.

These results provide evidence for the utility of methylation in phenotype imputation in general, and in biobank settings in particular. However, the promise of clinical translation of genomic risk scores, including PRS or MRS, is highly dependent on the clinical context of the patient. There is a large body of work investigating phenotype imputation and prediction in clinical settings using EHR data alone, typically with machine learning techniques, without any genomic data. To the best of our knowledge, the question of whether genomic data can be used to complement such algorithms has not been studied. Since the application of MRS or PRS to clinical data without taking into account the EHR data provides a limited clinical utility, this is a natural question.

Here, we demonstrate that MRS can be used in conjunction with EHR data to improve the imputation of clinical data of patients. Critically, most machine learning approaches rely on imputation because of the inability of such algorithms to process missing data, making accurate imputation a crucial step. We found that the combination of MRS with a gold standard imputation approach - SoftImpute [39 - for clinical data imputation, provides improved accuracy $\left(R^{2}\right)$ in $37.3 \%$ of the examined phenotypes with a median increase of $47.6 \%$. This result provides the potential to improve machine learning algorithms that use the EHR data, by complementing the data with methylation information for the patients.

In summary, our results quantify the contribution of methylation information in clinical settings, both in isolation and in conjunction with the EHR data, and they demonstrate the potential utility of epigenetic biobanks in clinical settings.

\section{Results}

Risk model description Analogous to the PRS 40, 41, we defined the MRS by a linear combination of $m \mathrm{CpG}$ site beta values $c$ and weights $w$ :

$$
\mathrm{MRS}=\sum_{i=1}^{m} w_{i} c_{i}
$$

To ensure the methylation risk score added predictive value over commonly captured features (e.g. age and sex), we created a baseline predictive model that included patients' age, sex, reference-based methylation cell-type composition estimates 42], self-reported race-ethnicity, self-reported smoking status, and the first ten genetic principal components 27] (see Supplementary Table S1 for cohort demographic data). We 


\begin{abstract}
fit the baseline model using a linear or logistic regression model depending on whether the outcome was continuous or binary. We compared the baseline model to models that included the baseline features as well as either methylation or genotype data. For both the MRS and PRS, we used regression with LASSO, elastic net, and ridge regularization over the genomic features while treating the baseline features as fixed covariates. We fit all models using 10 -fold double cross-validation, wherein each training set an additional cross-validation was performed for hyperparameter selection, then this training-set cross-validated model was used to predict the held-out test set. We tested for significance using an association test (via linear regression) between the cross-validated predicted outcome (i.e. the concatenated predictor across all folds) and the true outcome. For full details see Methods.
\end{abstract}

Methylation risk scores significantly outperform the baseline and PRS models From our EHR database, we extracted diagnosis codes, medication orders, and the most recent lab results, all of which occurred before the methylation samples were collected. We aggregated the ICD codes into higher-level phenotypes according to the phenotype code (Phecode) mapping proposed by Denny et al. [43. 44 and grouped individual medications by pharmaceutical subclass to increase generalizability and power.

We trained penalized linear models to predict clinical phenotypes for which there was a sufficient number of patient data available, which included 168 medication subclasses, 69 lab values, and 370 Phecodes. Using a Bonferroni-adjusted association test, the baseline and MRS models significantly imputed the usage of 69 and 88 medications, 18 and 33 labs, and 106 and 139 Phecodes respectively (Figure S1). We compared the performance of the MRS to a model that used both the PRS and baseline features on the same set of individuals, which significantly imputed the usage of 53 medications, 20 lab results, and 93 Phecodes. Notably, the baseline model imputed a greater number of medications and Phecodes than models that leveraged a PRS, which suggests that including genomic features may either add noise or our sample size may not have been sufficient to discover their effects for certain outcomes. We also show in Figure S2 that the baseline model gains some of its predictive power from genomicsderived features like ancestry PCs or estimated cell counts, and therefore a PRS or MRS may not offer a substantial improvement over these features for certain outcomes under the current sample sizes.

Next, we investigated outcomes for which genomics-based predictors add predictive power to the baseline features and, in such cases, the extent to which their inclusion improves predictive accuracy. On the outcomes for which the genomics-based predictors produced statistically significant imputations, we conducted a likelihood ratio test comparing an association test of the true outcome using the crossvalidated baseline predictor alone, to a model that included the cross-validated baseline predictor as well as the cross-validated predictor that included both baseline and genomic features (Methods). The methylation significantly improved the baseline predictor for 54 medications, 29 labs, and 56 Phecodes, and led to a median increase of $10.74 \%, 141.52 \%$, and $15.46 \%$ over the baseline predictor's accuracy (AUC, $R^{2}$ ) in each outcome, respectively (Figure 1). The genotypes significantly improved the baseline predictor for 8 medications, 3 labs, and 11 Phecodes, and led to a median increase of $18.42 \%$ over the baseline in the $R^{2}$ of the labs, but a median decrease of $1.75 \%$ and $0.94 \%$ in AUC of the medications and Phecodes respectively (Figure 1). We note that our internal sample size is likely underpowered to discover small genetic effects and therefore suggest the contributions made by the genotypes may be due to additional ancestry signal that was not captured by the first few genetic PCs.

The medications that improved the greatest using methylation corresponded to drugs often prescribed to individuals with neutropenia (hematopoeitic growth factors, AUC baseline .706 95\% CI $[.661, .748]$ to AUC methylation $.840[.807, .871]$ ) or chronic kidney disease (phosphate binder agents AUC from .731 $[.683, .777]$ to $.876[.842, .907])$. The lab panels best improved with the addition of the methylation-based predictor included those related to kidney function as well as cell counts (Urea nitrogen baseline adjusted $R^{2} .032[.007, .057]$ compared to .443 [.377,.509] with methylation, hemoglobin $.107[.063, .151]$ to $.289[.232, .346])$. The addition of the genotype-based predictor improved the imputation of hematocrit (adjusted $R^{2}$ from $.077[.041, .114]$ to $.092[.052, .132]$ ) and total protein (adjusted $R^{2} .094[.047, .141]$ to $\left..111[.060, .162]\right)$, both of which are influenced by ancestry [45, 46]. In the context 

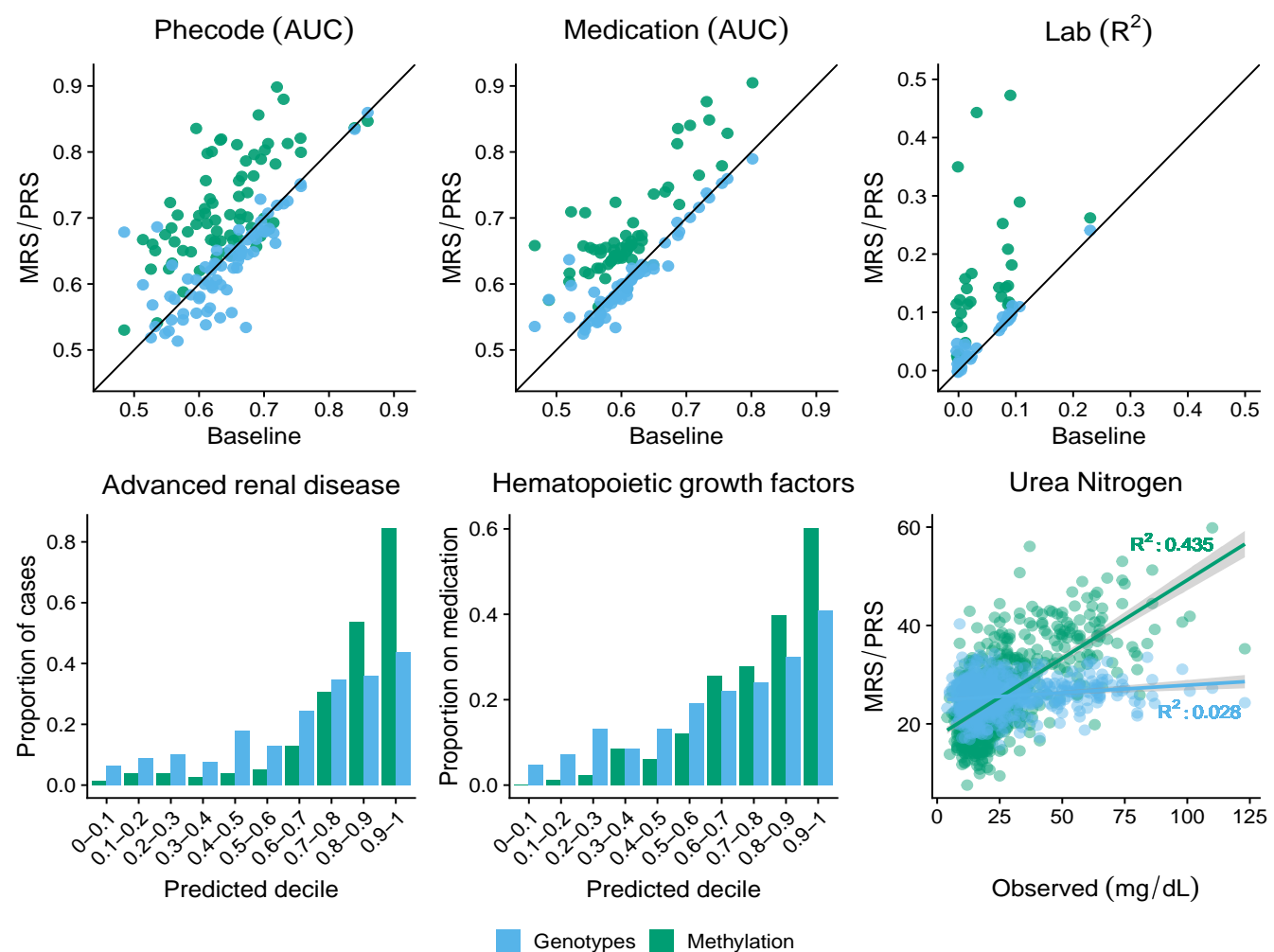

Figure 1. MRS increases imputation accuracy on a variety of outcomes (Top) The performance of the PRS (blue) and MRS (green) imputations on the y-axis with the baseline model performance on the $\mathrm{x}$-axis. The performance of binary phenotypes (Phecodes, medications) is measured using area under the ROC curve (AUC) and the performance of continuous phenotypes (lab results) is measured using proportion of variance explained $\left(R^{2}\right)$. Shown is the performance on the union of outcomes that were significantly improved over the baseline model by either the MRS or PRS and that were significantly imputed their corresonponding predictor (72 Phecodes, 59 medications, and 31 labs). (Bottom) The disease incidence as a function of the PRS (blue) and MRS (green) binned by deciles (left, middle); and the observed Urea Nitrogen lab result value plotted against its imputed value (right).

of Phecodes, methylation greatly increased the imputation of advanced renal disease over the baseline and genotype models (for example, AUC baseline $.720[.673, .762]$ to $0.898[.867, .927]$ with methylation), and the genotype model increased the imputation of actinic keratosis (AUC from .694 [.631,.747] to .728 $[.672, .784])$.

Overall, when looking at the intersection of medications significantly imputed by either the methylation and genotypes or methylation and baseline, $92 \%$ were better imputed by methylation sites than genotypes (median $9.13 \%$ increase) and $78 \%$ were better imputed by methylation compared to the baseline (median $6.81 \%$ increase). Methylation improved the baseline imputation accuracy by over $15 \%$ for 14 medications. In the context of significantly imputed lab values, methylation explained more variability than the baseline (median 398\% increase) and genotype (median $274 \%$ increase) predictors in $97 \%$ and $94 \%$ of the respective union of significantly imputed labs. For 22 labs, the percent increase of imputation accuracy was greater than $15 \%$ over the baseline model. Methylation was more accurate than the baseline (median $3.48 \%$ increase) or genotypes (median $6.58 \%$ increase) for $70 \%$ and $83 \%$ of each respective union of Phecodes. For 29 Phecodes, the methylation offered over a $15 \%$ increase in predictive accuracy compared to the baseline model. For a substantial proportion of outcomes, the MRS predictor added 
statistically significant predictive value over the PRS predictor (Figure S3). This was generally not true when comparing whether the PRS added predictive value over the MRS. For the imputation performance on the full list of phenotypes, see Supplemental Tables S2, S3, and S4. To see the number of CpGs selected for each MRS, see Supplemental Tables S5, S6, and S7.

Importantly, cell-type composition, age, sex, BMI, smoking status and ancestry provide sufficient power for the imputation of many EHR outcomes. We show explicitly in Supplementary Figure S4 that genomics derived features such as cell-type composition and ancestry PCs likely contribute to accurate imputation of several outcomes. In our analyses, we directly compared the power gained by methylation over the aforementioned set of baseline features. However, we note that obtaining these baseline features may be unnecessary as the methylation alone may capture their signal $27,30,32,47,48$. Further, previous reports have suggested that approaches that fit all methylation probes simultaneously with regularization may perform better when excluding latent confounders, such as cell type composition [49]. We therefore suggest that using the methylation alone is sufficient to replicate a substantial proportion of the associations generated from the baseline features alone.

Using methylation risk scores improves imputation approaches Due to significant heterogeneity in patient populations, the diagnosis and treatment process can vary widely between patients, causing many variables to be left unobserved. This sparse structure in the data must be reconciled before performing many downstream analyses, and the imputation accuracy of these unobserved variables is therefore crucial to subsequent steps. A commonly-used approach for imputation is matrix completion, for example, SoftImpute [39, where the data matrix is reconstructed from a low-rank representation. Often, one would jointly use demographic information, diagnosis codes, lab results, and medications to generate an estimate of the unobserved EHR values using an imputation method such as SoftImpute, and therefore we used this as our baseline imputation estimate 50 .

To investigate whether methylation can add additional useful information to the imputation, we included the MRS values as part of imputation procedure and compared the performance to the estimates that do not take methylation data into account (see Methods). Specifically, we included cross-validated MRS values for diagnosis codes, lab results, medications, and demographics that were significantly imputed as 261 additional features (i.e. columns of the input matrix) in the imputation procedure. We randomly removed a subset of the observed lab results, including other labs that are ordered as part of the same lab panel(s), and imputed the masked values using the remaining observed values. The imputed values were then compared to the held-out, masked values to assess the quality of the imputation. In Figure 2, we show the imputation accuracy $\left(R^{2}\right.$ between the masked true and imputed values) for labs where the addition of cross-validated MRS to the baseline SoftImpute procedure explained significantly more variability. Of the 67 lab results considered, $25(37.3 \%)$ were significantly better imputed by including the MRS values. Including the MRS values led to a median increase of $47.6 \%$ (95\% CI 17.3\%-90.9\%) in the imputation $R^{2}$ values.

Methylation risk scores will improve with larger sample sizes In this study, our analyses of imputation accuracy were performed on 831 individuals' methylation and genetic features. For many phenotypes, the genetic effects are relatively small and require large sample sizes to identify associations between genomic features and the outcome of interest. Consequently, in many biobanks the number of individuals with measured genomic features is several orders of magnitude larger than our sample size [1-3. While the methylation data provided sufficient power to significantly impute numerous outcomes, there may remain much power to be gained by increasing the number of methylation samples to numbers approaching biobank-scale.

To determine the role of sample size in our imputation accuracy, we performed an experiment in which we downsampled the number of individuals in our data and trained models on the subsampled data. From the set of outcomes most accurately imputed by methylation and that also significantly improved the baseline's imputation, we chose 10 medications, labs, and Phecodes on which to perform 10-fold cross- 


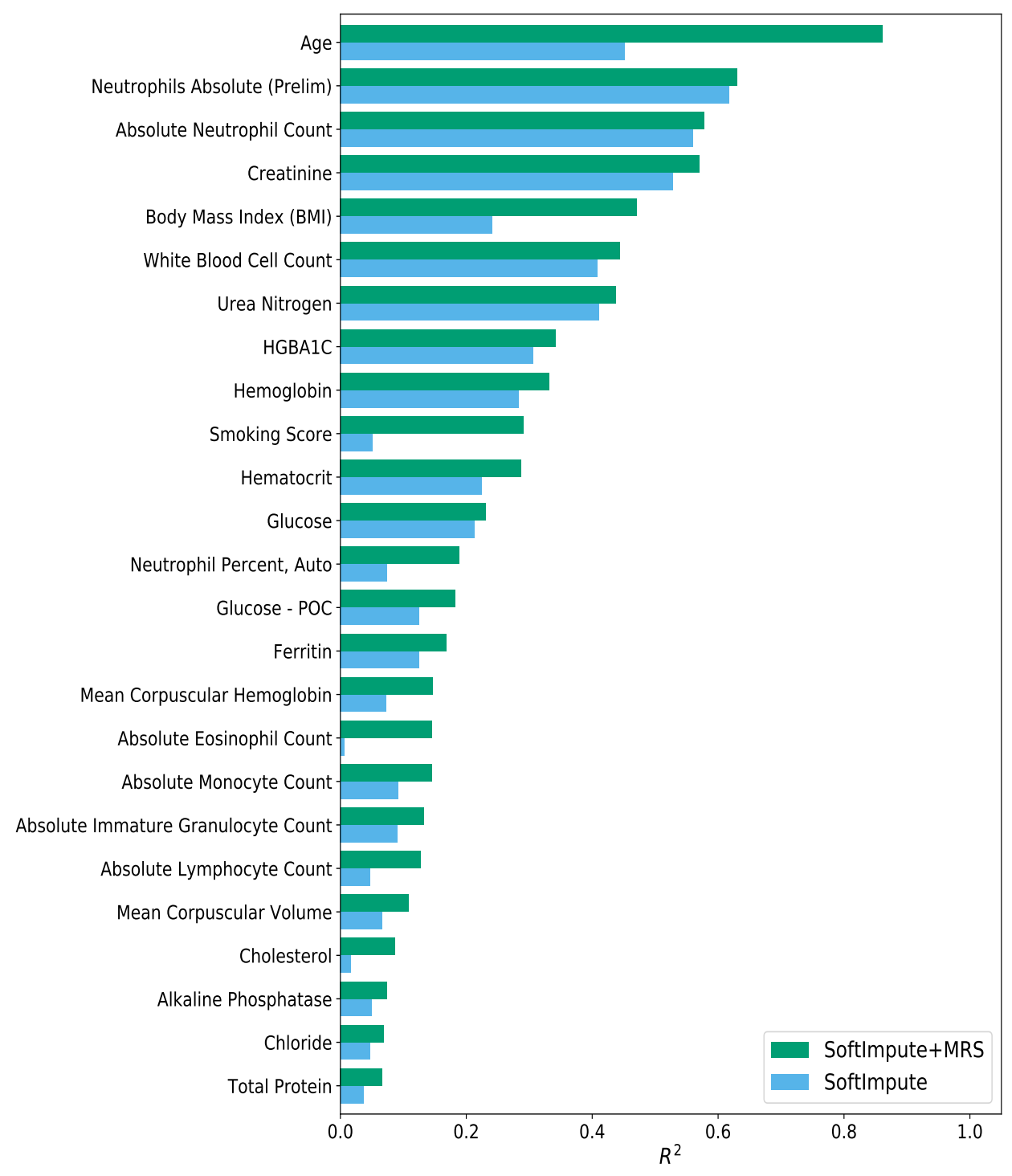

Figure 2. Improvement in lab result imputation performance by including MRS For lab results that were significantly better imputed using a matrix completion imputation procedure that included the MRS values, we compare the quality of the imputed values $\left(R^{2}\right)$ using only the EHR data (SoftImpute) to the values generated when including the MRS values in addition to the EHR data (SoftImpute+MRS).

validation. For each sample size, we repeated the procedure 20 times to attempt to mitigate variance due to ascertainment effect. Though we selected features that had high accuracy using the full set of data, our results suggest that our models may become more accurate as the sample size increases (Figure 3. Figures S6 S7/S8]. We further posit that there may be additional outcomes that will be significantly imputed as the number of methylation samples increases.

Comparing MRS to UKBiobank PRS As expected, due to a small sample size and the likely small effects of SNPs on phenotypes, the PRS developed using the UCLA cohort did not add substan- 

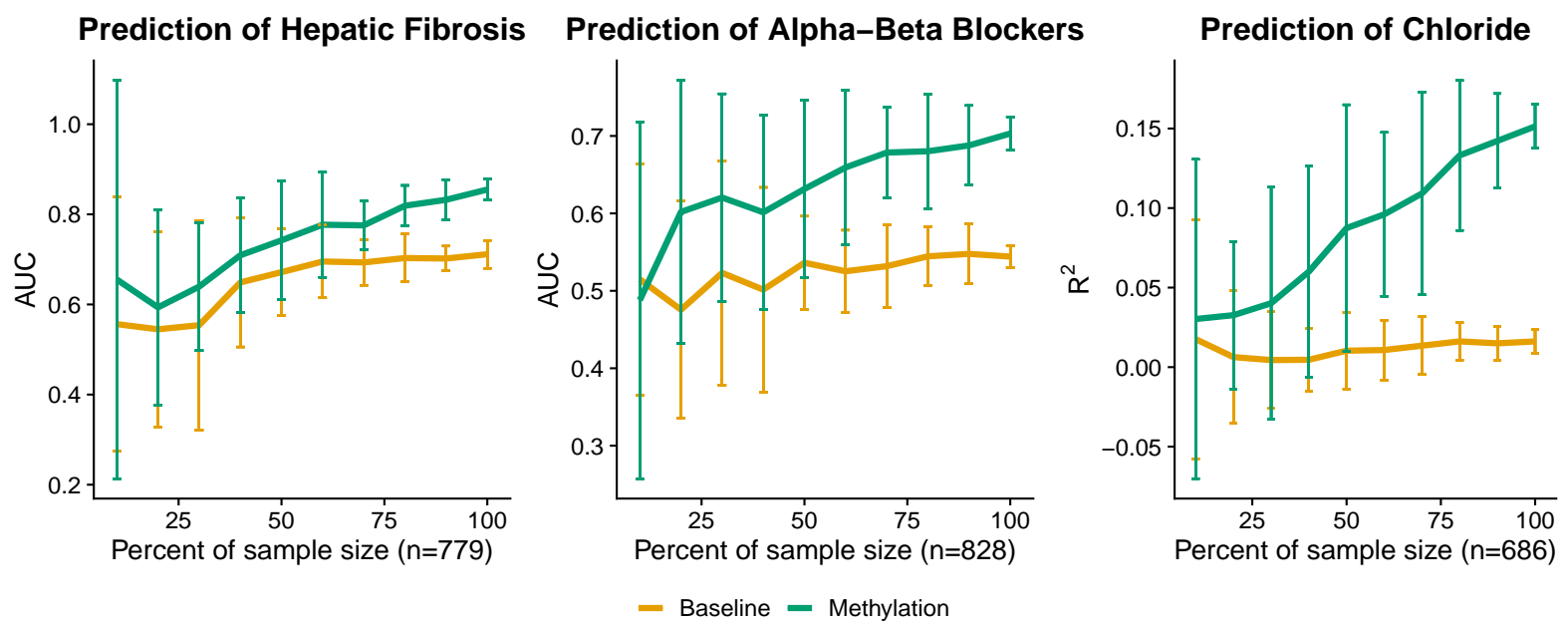

Figure 3. Imputation accuracy may improve with additional samples We downsampled the number of individuals to evaluate the imputation performance as a function of sample size using a wellimputed medication, lab value, and Phecode. The performance is significantly affected by the number of individuals, suggesting that there is additional power to be gained with the addition of more methylation samples.

tial predictive power over the baseline features. Studies leveraging biobanks with sample sizes several magnitudes larger than the cohort at UCLA however, have shown non-zero heritability for a variety of phenotypes [1, 51 53. Therefore, we sought to compare the MRS and PRS generated with the UCLA data to a polygenic risk score created using the UKBiobank data [54. To do so, we obtained the genotype weights corresponding to 10 polygenic risk scores trained on the UKBiobank (Table S10 [52 55] data and imputed the external risk scores into our health record system using PLINK [56. We included in the comparison labs that were significantly imputed by the baseline model and excluded labs that corresponded to cell counts or labs for which the internal PRS outperformed the external PRS (indicating a mismatch in the phenotypes or cryptic population structure that was unaccounted for by principal components). While the external polygenic risk score improved substantially the imputation performance relative to the internal polygenic risk score, it did not significantly outperform the methylation for any of the tested phenotypes (Figure 4). The methylation remained the best predictor in general - even when trained on fewer than 1000 samples - significantly outperforming the other models in the imputation of urea nitrogen, creatinine, hemoglobin, hematocrit, and albumin. The externally-derived polygenic risk score greatly outperformed both the internally-derived PRS and the MRS when predicting glycated hemoglobin (HGBA1C) and HDL levels, however the improvement was not significant. For detailed information on the external PRS and accession numbers, see Supplementary Table S10.

Similarly to the analyses in which we examined whether predictors that leverage genomics offered predictive value over the baseline predictor, we examined whether our internal MRS and the externally trained PRS offer information that is complementary to the other. To do so, we measured the accuracy when using the MRS, external PRS, both risk scores, and as well as both risk scores and their interaction on the same set of labs as our original analysis. None of the models significantly outperformed the MRS alone (Figure S10). However, there was a significant interaction effect between the MRS and external PRS on creatinine ( $\mathrm{p}=9.16 \mathrm{e}-05)$, as well as a nominally significant interaction effect on mean corpuscular hemoglobin $(\mathrm{p}=1.45 \mathrm{e}-02)$. As the interaction terms improved the accuracy for both outcomes, there may be added value in leveraging both MRS and PRS for imputation tasks, especially those that take advantage of non-linear effects. 


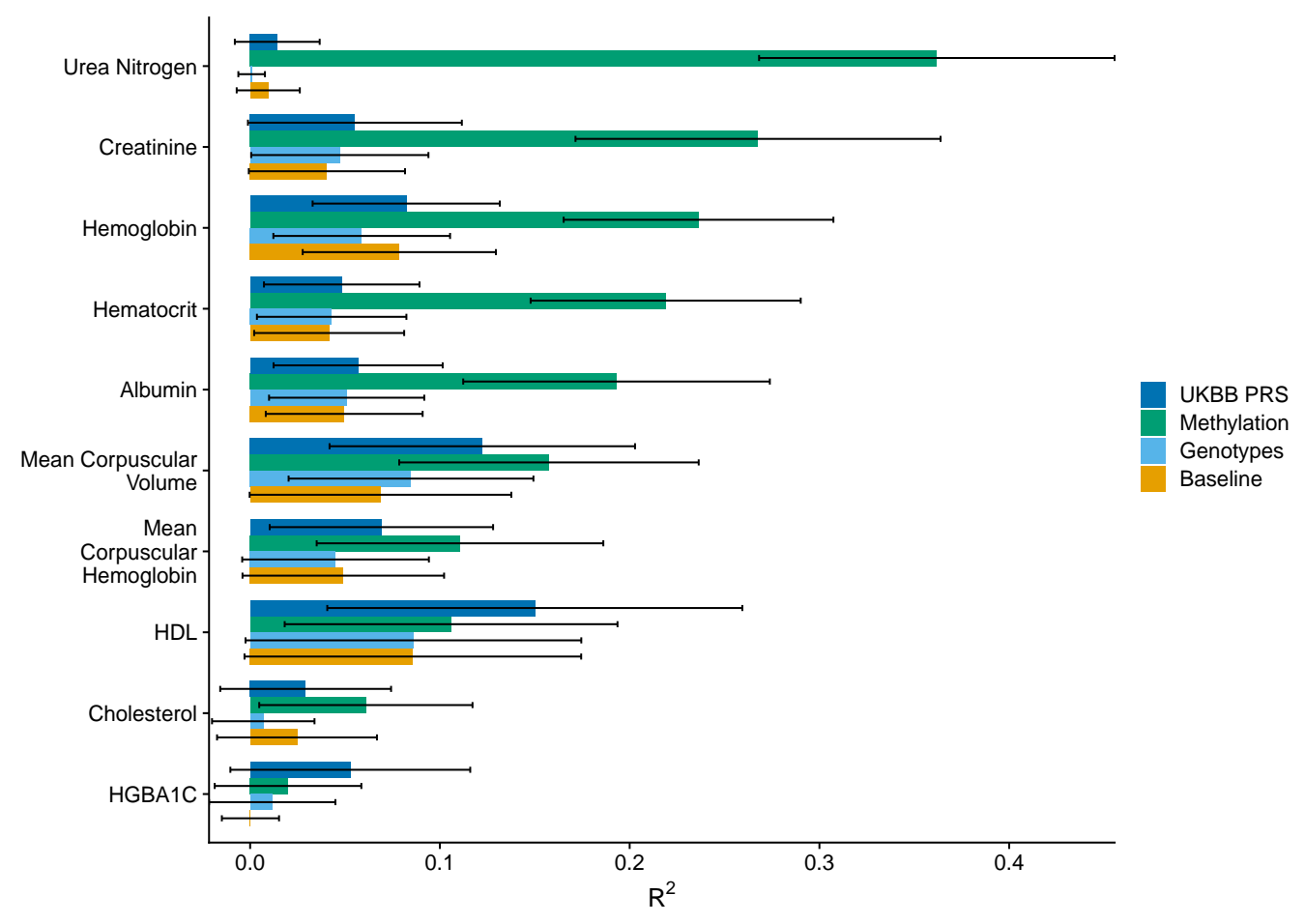

Figure 4. Labs as imputed by methylation, genotypes, and an externally-trained polygenic risk score The cross-validated $R^{2}$ between the true and imputed lab value on 541 unrelated patients of non-Hispanic-Latino white-identifying individuals using a baseline predictor as well as a baseline predictor with methylation, genotypes, and a PRS externally-trained from UKBiobank summary statistics. HDL corresponds to high-density lipoprotein cholesterol and HGBA1C to glycated hemoglobin.

Evaluation of methylation risk scores across ancestral populations Previous reports have suggested that a significant confounder to the application and versatility of polygenic risk scores is population structure, where a population-specific bias is induced that affects generalizability of PRS to different ancestries [57 59]. The collection of samples analyzed throughout this study is ethnically heterogeneousindividuals were self-identified as non-Hispanic/Latino European, Hispanic/Latino, Black, or Asian. Methylation data is also influenced by differences in population [60], and in particular the first several methylation principal components sufficiently capture population structure in European and African groups 61,62$]$ ). Consequently, we examined the performance of the methylation risk scores within and across ancestral populations.

Primarily, after training the models on the entire heterogeneous set of samples, we examined the predictive performance within each ancestral population. When we examined the top 10 best-imputed (by MRS across the entire set of individuals) lab panels, medications, and Phecodes, only 10 of the entire 180 possible comparisons $\left(\begin{array}{l}4 \\ 2\end{array}\right)$ comparisons across 30 outcomes) displayed significant differences between the predictive performance within each population separately (Figures 5 , S11, S12.

In a second replication analysis we trained predictive models within ancestral groupings separately. As the individuals self-identified as either Black or Asian comprised less than 100 individuals in both groupings, we focused our analyses on Hispanic/Latino- and white-non-Hispanic/Latinoidentifying individuals. We retrained models for the top 5 best-imputed (by MRS) medications, lab panels, and unique Phecodes on the Hispanic/Latino individuals and white non-Hispanic/Latino individuals alone and treated a prediction as significant if its association p-value was lower than .01. Creatinine, 


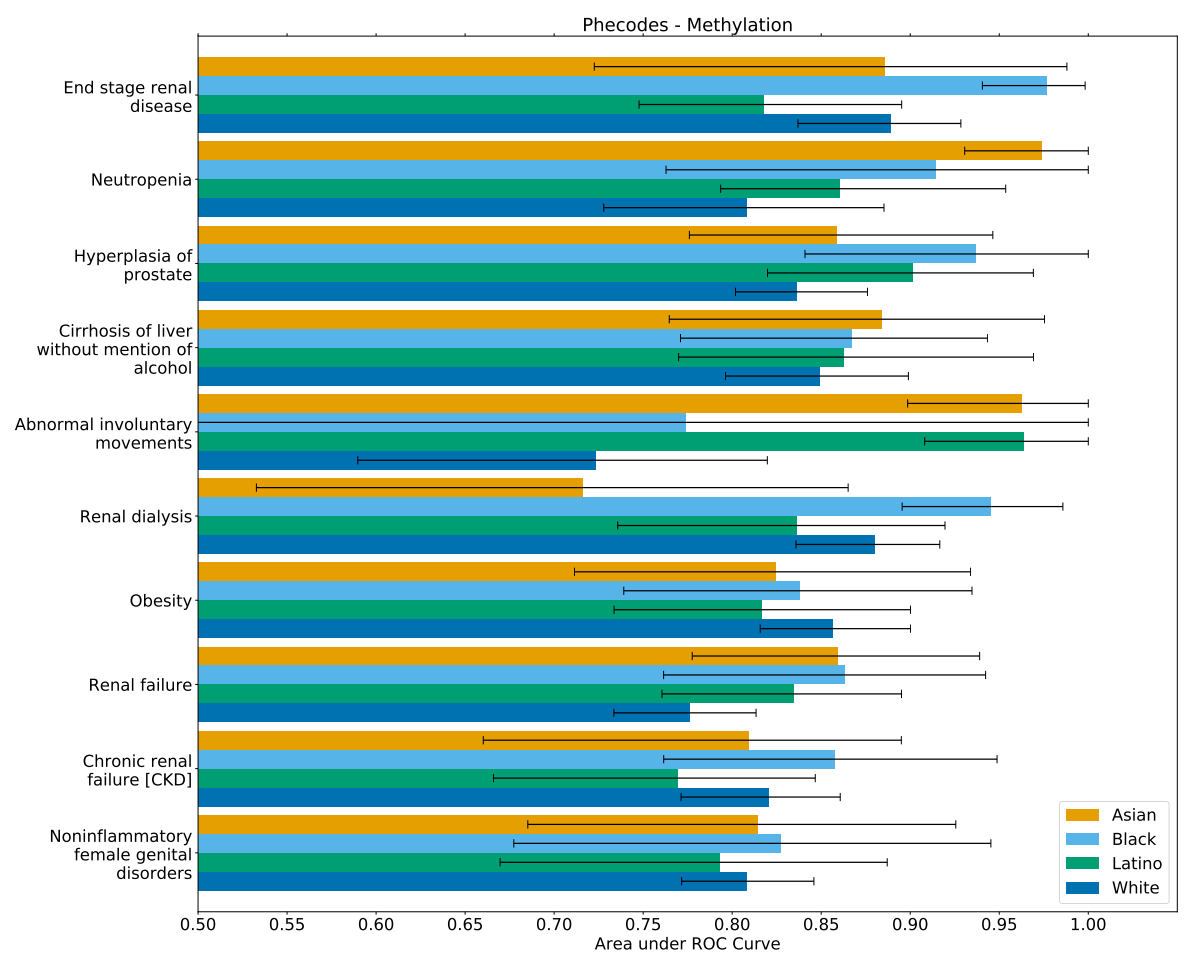

Figure 5. Best methylation-imputed Phecodes within ancestral populations. After training a model on the entire heterogeneous population of individuals, we evaluated the predictive performance within each population separately. We observed only 6 (of 60) significant differences between self-reported ancestral groupings.

hemoglobin, and urea nitrogen replicated across both groupings, however, hematocrit and mean corpuscular hemoglobin did not replicate in the Latino/Hispanic grouping (Table 1). In the context of medications, CMV agents, osmotic diuretics, phosphate binder agents, hematopoietic growth factors, and immunosuppressive agents replicated within the white non-Hispanic/Latino population but only CMV and immunosuppressive agents replicated within the Hispanic/Latino population (Table 1). Finally, Phecodes corresponding to immunity deficiency, hypertensive renal disease and end-stage renal failure replicated within both groupings, however, neutropenia and anemia replicated only within the white non-Hispanic/Latino set of individuals (Table 1).

Replication of methylation risk scores across external datasets To evaluate the transferability of the MRS to a different population, we performed several experiments in which we imputed the MRS into external datasets. Primarily, we focused on imputation of kidney-related outcomes as they were the most accurately imputed in our own cohort. To do so, we leveraged a dataset that used the HumanMethylation27k array to measure the methylation of 194 individuals who had Type 1 Diabetes, $49.7 \%$ of whom had nephropathy (cases) 63. We re-trained the models for a Phecode corresponding chronic renal disease as well as labs corresponding to creatinine and urea nitrogen on our in-house data, limiting our analysis to the 27,000 sites that belonged to the external dataset. The imputed chronic renal disease was significantly associated with nephropathy in the external dataset $(\mathrm{p}=8.32 \mathrm{e}-05, \mathrm{AUC}=.684[.615, .758]$. Further, both of the imputed values for creatinine and urea nitrogen were significantly associated with nephropathy $(\mathrm{p}=5.11 \mathrm{e}-07, \mathrm{AUC}=.739[.670, .808]$ and $\mathrm{p}=3.71 \mathrm{e}-05 \mathrm{AUC}=.693[.619, .767]$, respectively). Importantly, when limiting our internal analysis to sites only on the $27 \mathrm{k}$ array, the association signal 


\begin{tabular}{|l|c|c|c|c|}
\hline Outcome & Metric & $\begin{array}{c}\text { Accuracy, p-value } \\
\text { Hispanic/Latino } \\
(\mathbf{n = 1 1 8})\end{array}$ & $\begin{array}{c}\text { Accuracy, p-value } \\
\text { white, non- } \\
\text { Hispanic/Latino } \\
\mathbf{( n = 5 4 3 )}\end{array}$ & $\begin{array}{c}\text { Accuracy, p-value } \\
\text { all ethnicities } \\
\mathbf{( n = 8 3 3 )}\end{array}$ \\
\hline Creatinine & $R^{2}$ & $.217,4.63 \mathrm{e}-07$ & $.356,7.47 \mathrm{e}-46$ & $.457,1.27 \mathrm{e}-95$ \\
Hematocrit & $R^{2}$ & $.045,2.91 \mathrm{e}-02$ & $.188,1.87 \mathrm{e}-21$ & $.246,1.14 \mathrm{e}-42$ \\
Hemoglobin & $R^{2}$ & $.096,1.21 \mathrm{e}-03$ & $.204,2.54 \mathrm{e}-23$ & $.283,3.02 \mathrm{e}-50$ \\
Mean corpuscular hemoglobin & $R^{2}$ & $.050,2.12 \mathrm{e}-02$ & $.122,9.70 \mathrm{e}-14$ & $.208,7.04 \mathrm{e}-35$ \\
Urea nitrogen & $R^{2}$ & $.289,2.97 \mathrm{e}-09$ & $.349,7.61 \mathrm{e}-44$ & $.435,2.50 \mathrm{e}-87$ \\
CMV Agents & $\mathrm{AUC}$ & $.874,9.27 \mathrm{e}-07$ & $.875,3.47 \mathrm{e}-16$ & $.905,1.72 \mathrm{e}-38$ \\
Osmotic Diuretics & $\mathrm{AUC}$ & $.530,0.841$ & $.842,2.27 \mathrm{e}-12$ & $848,6.37 \mathrm{e}-34$ \\
Phosphate binder agents & $\mathrm{AUC}$ & $.608,0.321$ & $.819,7.76 \mathrm{e}-17$ & $.876,1.11 \mathrm{e}-50$ \\
Hematopoietic growth factors & $\mathrm{AUC}$ & $.567,0.476$ & $.780,1.51 \mathrm{e}-19$ & $.840,1.75 \mathrm{e}-45$ \\
Immunosuppressive agents & $\mathrm{AUC}$ & $.721,1.43 \mathrm{e}-04$ & $.823,6.36 \mathrm{e}-22$ & $.828,9.44 \mathrm{e}-41$ \\
Neutropenia & $\mathrm{AUC}$ & $.689,5.60 \mathrm{e}-02$ & $.800,7.68 \mathrm{e}-10$ & $.836,1.11 \mathrm{e}-19$ \\
Immunity deficiency & $\mathrm{AUC}$ & $.889,4.06 \mathrm{e}-09$ & $.818,3.26 \mathrm{e}-19$ & $.821,9.74 \mathrm{e}-33$ \\
Anemia & AUC & $.637,9.75 \mathrm{e}-02$ & $.698,3.13 \mathrm{e}-08$ & $.789,1.40 \mathrm{e}-32$ \\
Hypertensive renal disease & AUC & $.715,1.35 \mathrm{e}-04$ & $.688,6.74 \mathrm{e}-10$ & $.801,1.45 \mathrm{e}-42$ \\
End-stage renal failure & AUC & $.677,1.80 \mathrm{e}-03$ & $.868,2.51 \mathrm{e}-29$ & $.898,5.46 \mathrm{e}-72$ \\
\hline
\end{tabular}

Table 1. Replication statistics within ethnic groupings. Predictive accuracy $\left(R^{2}\right.$ and AUC) for MRS trained within only Latino/Hispanic- or white-non-Latino/Hispanic-identifying individuals compared to the accuracy trained on the entire, cross-ethnic cohort.

decreased (for chronic renal disease from $\mathrm{p}=6.81 \mathrm{e}-51$ to $\mathrm{p}=3.13 \mathrm{e}-29$, creatinine $\mathrm{p}=1.27 \mathrm{e}-95$ to $\mathrm{p}=3.14 \mathrm{e}-$ 62 , and urea nitrogen $\mathrm{p}=2.50 \mathrm{e}-87$ to $\mathrm{p}=8.44 \mathrm{e}-34$ ). However, likely due to correlation between CpGs, the association tests for outcomes trained on the smaller set of sites were still significant.

Second, we expanded our replication analyses to include phenotypes that were unrelated to kidney function. In these analyses, we revisited epigenome-wide association studies (EWAS) of Schizophrenia [64 and Rheumatoid Arthritis 65] and imputed commonly prescribed medications for each dataset-for Schizophrenia we used phenothiazines, and for Rheumatoid Arthritis we used glucocorticosteroids. To ensure our MRS captured medication intake status and were not merely serving as proxies for the disease, we re-trained our models while conditioning on the trait of interest. The imputed phenothaizine intake was significantly associated with Schizophrenia case-control status $(\mathrm{p}=8.71 \mathrm{e}-04, \mathrm{AUC}=.568[.527, .611])$ and the imputed glucocorticosteroids usage was significantly associated with Rheumatoid Arthritis casecontrol status $(\mathrm{p}=2.72 \mathrm{e}-07, \mathrm{AUC}=.626[.584, .669]$. Weights for both medications were trained on CpGs corresponding to those present on the HumanMethylation450k array and also included their corresponding disease in the baseline set of covariates. Accordingly, the association signal of phenothiazines dropped from 1.14e-07 to 3.99e-05 and the performance of glucocorticoids dropped from 1.35e-16 to $1.82 \mathrm{e}-15$ when compared to the MRS trained on the set of EPIC array CpGs and with the baseline features as covariates. 


\section{Methods}

Electronic Health Record Data De-identified electronic health record data for this study was extracted from the perioperative data warehouse (PDW), a custom-built, robust data warehouse containing all patients who have undergone surgery at UCLA Health since the implementation of UCLA's EMR (EPIC Systems, Madison, WI, USA) in March 2013. The PDW, which has been described previously [66], has a two-stage design. First, data are extracted from EPIC's Clarity database into 29 tables organised around three distinct concepts: patients, surgical procedures, and health system encounters. Then, these data are used to populate a series of 4000 distinct measures and metrics such as procedure duration, admission ICD codes, lab results, and medication orders.

Patient Ascertainment Methylation and genotype samples were collected using blood from 831 patients as part of the UCLA ATLAS precision health initiative between October 26, 2016 and December 10, 2018 67]. The samples were collected from patients before undergoing surgery with general anesthesia at UCLA Health, and the patients had not undergone surgery in the 30 days prior to blood sample collection. Of these patients, 302 were selected for inclusion based on the presence of acute kidney injury (AKI), defined as an Acute Kidney Injury Network (AKIN) classification of one or greater, after undergoing surgery. An additional 348 patients were risk-matched controls, with either glomerular filtration rate (GFR) less than or equal to 38 (210 patients), or GFR greater than 38 and a propensity risk score that matched case patients (348 patients). The propensity score was created using available EHR features such as age, weight, BMI, and other preoperative features that were measured in the hospital. Within the control group, we also performed a similar procedure ascertained on whether individuals were a heart attack case. Controls for heart attack patients were also selected using propensity scoring. Demographics of the patient population are further described in Table S1.

Medication Usage For each medication, a patient was labeled as using a medication if the electronic health record contained a medication order that occurred before the methylation sample collection date. Medications were grouped by pharmaceutical subclass using the Generic Product Identifier (GPI) hierarchical classification system codes. Any medications that were ordered in fewer than $5 \%$ of the patients were excluded from the analysis. In total, 168 pharmaceutical subclasses were considered in our analysis. The number of patients using medications from each subclass is shown in Supplemental Table S8. In Supplemental Table S9, we show for each pharmaceutical subclass the specific medication that patients in our cohort received.

Lab Results The most recent lab result prior to the methylation sample collection was extracted from the PDW for each patient. Any labs with a result date that occurred more than 365 days before the methylation sample collection date were excluded from the analyses. Additionally, labs for which there were less than 50 patients with valid results were excluded. We were left with a total of 69 lab values on which to run our models.

Diagnosis Codes International Classification of Diseases, Ninth Revision (ICD-9) and International Classification of Diseases, Tenth Revision (ICD-10) codes are a standard set of diagnosis codes, primarily used for billing purposes. While these codes provide a standardized methodology for describing a diagnosis, they are very specific. To map these specific diagnosis codes into meaningful, distinct diseases/traits, Denny et al. aggregated the ICD codes into phenotype codes (Phecodes) 43,44 . Specifically, for each patient, we queried all diagnoses prior to the methylation sample collection date, and used the Phecode (version 1.2) mapping to aggregate ICD-9 and ICD-10 codes to unique, meaningful phenotypes. If a patient's diagnosis record had both ICD-9 and ICD-10 labels, the ICD-10 to Phecode mapping was used instead of the ICD-9 to Phecode mapping. Each Phecode was treated as a binary variable, indicating the presence or absence of a relevant diagnosis code at any point in time before sample collection. We 
excluded rare Phecodes (occurrence less than $5 \%$ of the patients) and, in total, our cohort contained 370 unique Phecode phenotypes.

Preprocessing of genotype data for cross-validation We measured the genotypes for 831 individuals based on their DNA sampled from whole blood using the ATLAS genotype array. We preprocessed the genotype data using Beagle (d20) [68], PLINK (1.07) [56], and GCTA (1.93.2) [69]. We restricted the genotypes to autosomal variants, removed rare variants $(\mathrm{MAF}<.05)$, and filtered for variants that met Hardy-Weinberg equilibrium with p-value threshold $10^{-6}$. We also removed individuals and variants with more than $1 \%$ missing values. For the purpose of running cross-validation, we used Beagle to impute only any remaining missing values, but did not impute to an external dataset. We show that with our sample size and phenotypes evaluated, using genotypes imputed to an external reference does not significantly improve our results (Figure S14). In total we were left with 292,808 SNPs. To obtain principal components, we ran PCA using plink on the chipped genotypes.

Preprocessing and imputation of genotype data for comparison to external models We used a version of the ATLAS genotype data that was imputed to an external dataset, as detailed in 67. Briefly, after performing quality control, genotypes were uploaded the Michigan Imputation Server 70 . The server phases the genotype data using Eagle v2.4 71] and performs imputation using the TOPMed Freeze5 imputation panel [72] using minimac4[73]. We applied the same quality control and filters to the imputed genotypes as we did the chipped genotypes, and we were left with a total of 5,574,956 SNPs.

Preprocessing of methylation array data We measured methylation data for 831 individuals based on their DNA sampled from whole blood using the EPIC Illumina array. To generate beta-normalized methylation levels at each CpG, we ran the default pipeline of ENmix (1.22.0) 74] on the the raw probe data (IDAT files), which performs background correction, RELIC dye bias correction, and RCP probetype bias adjustment. We removed from our analysis CpGs that coincided with SNP loci as well as CpGs on the sex chromosome. We also filtered out outlier samples, defined as having a PC score more than 4 standard deviations away from the average PC score in the first two principal components. In the imputation tasks, we removed sites with low variability (standard deviation $<0.02$ ) leading to a total of 269,471 sites.

Imputation using baseline medical features To establish a baseline level of imputation performance, we constructed a set of features derived from basic patient information. We trained a simple linear (or logistic) model with 10-fold cross validation using an intercept and patients' age, sex, BMI, methylation-based cell-type proportions (from the reference-based method of Houseman et al. [42]), selfreported ancestry, first ten genetic principal components, and smoking status (never, former or current). Importantly, we wished to establish how well an outcome (medication, Phecode, or lab value) could be imputed by using covariates (e.g. ancestry, age, smoking status) that are known to be captured by genomics.

Imputation using a single penalized linear model After establishing a baseline level of imputation performance, we performed penalized logistic and linear regression using either individuals' methylation, genotypes, or both. More concretely, we fit 10-fold cross-validation using LASSO, elastic net and ridge regularization under the following two models:

$$
\begin{aligned}
& y=\alpha_{G}+G \beta_{G}+C \beta_{C}+\varepsilon_{G} \\
& y=\alpha_{M}+M \beta_{M}+C \beta_{C}+\varepsilon_{M}
\end{aligned}
$$

where $y$ corresponds to the outcome, $\alpha$ the model-specific intercept, $G$ the $n \times s$ genotypes, $M$ the $n \times c$ methylation data, $\beta$ the vector of length- $s$ or $-c$ effect sizes for the given explanatory variable, 
$C$ and $\beta_{C}$ the covariates from the baseline model and their corresponding effect sizes, and $\varepsilon$ the length $n$ noise vector. We refer to models (2) and (3) as the PRS and MRS respectively, and note that they also include the baseline features. After fitting all three penalized linear models for a given datatype and outcome, we selected a final model as determined by the model with the highest cross-validated metric (AUC or $R^{2}$ if the outcome was binary or continuous, respectively). We fit all penalized models using package bigstatsr 41 . We share MRS weights for outcomes that were significantly imputed at https://github.com/cozygene/EHR_MRS_UCLA. We also include details on the number of CpGs selected for each MRS in these analyses in Supplemental Tables S5, S6, and S7.

Imputing lab results using EHR data and MRS values with softImpute Imputing a partiallyobserved matrix of values is often formulated as a matrix-completion problem. In a matrix completion problem, the observed values of the matrix are used to estimate the values of the unobserved values by assuming that there is some underlying structure that is responsible for generating the data. For example, in the popular SoftImpute method [39], the data is assumed to be well-approximated by a lowrank representation, and the error between the observed values and the reconstructed values is minimized through a convex optimization procedure. However, since the unobserved values are, by definition, not observed, and therefore cannot be used to assess the imputation performance, the primary method for measuring the performance involves masking (removing) observed values and comparing the imputed values to these held-out, true values.

The EHR data used in the imputation procedure included demographic information, diagnosis codes, medication usage, and lab results, which were extracted from the EHR database using the previously described criteria. In addition to the EHR data, we also ran the imputation procedure while including relevant MRS values. Specifically, we included the MRS values for demographics, diagnosis codes, medication usage, and lab results that were imputed at a statistically significant level. These MRS values were added as additional observed features to the EHR matrix.

To estimate the imputation performance, we randomly masked $10 \%$ of the observed lab result values, and performed the imputation procedure (SoftImpute matrix completion) to generate estimates of the missing values. However, since labs are most often ordered in panels, for example a metabolic panel, if a lab is missing then typically other labs that are part of the same panel are also missing. We simulated a more realistic missingness scenario by, instead of masking out values only from a specific lab $l$, masking out all labs that are ordered as a panel that include lab $l$. This masking procedure was done per lab, using 10-fold cross-validation, such that $10 \%$ of the non-missing values of a particular lab result (and its associated lab panels) were masked (removed), and the remaining $90 \%$ of the observed values were used to complete the matrix. Matrix completion was performed using the SoftImpute algorithm, as implemented in the fancyimpute [75] python package (version 0.5.5). The proportion of variance explained $\left(R^{2}\right)$ of the true lab values by the imputed lab values was used to measure the imputation performance. Confidence intervals were derived using bootstrapping.

Hypothesis testing To determine whether an imputation was significant or whether one predictor offered significant additional explanatory signal, we conducted our hypothesis tests using a linear (logistic) regression framework. Primarily, after running cross-validation or generating a single predictor $\hat{y}$ for an outcome $y$, we would test whether the imputation was significant by comparing it to $y$ :

$$
y=\alpha+\hat{y} \beta+\varepsilon
$$

Where Equation (4) corresponded to linear regression when the outcome was continuous, and logistic regression when the outcome was binary, $\alpha$ was the intercept, and $\beta$ was an effect size indicating association of the predictor with the outcome. Notably, by building our testing framework as a linear model, we can easily extend it to include additional predictors in order to test whether the additional predictors significantly improve the fit of the regression - or more simply, whether predictor $\hat{y}_{j}$ offers additional predictive power over $\hat{y_{i}}$ by conducting a likelihood ratio test of the following nested models: 


$$
\begin{aligned}
& y=\alpha_{i}+\hat{y}_{i} \beta_{i}+\varepsilon_{i} \\
& y=\alpha_{i j}+\hat{y}_{i} \beta_{i}+\hat{y}_{j} \beta_{j}+\varepsilon_{i j}
\end{aligned}
$$

Where $i$ and $j$ index either the baseline, MRS, or PRS models. We corrected for multiple hypothesis tests within each outcome and method by using a Bonferroni adjustment at $\alpha$ level .05 .

Imputing external polygenic risk scores into the ATLAS cohort We compared our in-house built risk scores to risk scores learned in the UKBiobank dataset 51, 53. In both 51, 53 the authors construct their PRS using penalized regression akin to as we have done in our analyses. Notably, using penalized regression on individual-level genotypes allows one to automatically, optimally control for shrinkage and variable selection at the step of model generation 41,76 . This is in contrast with many commonly used polygenic risk score tools such as LDPred 77] or PRSCS[78, that attempt to perform shrinkage or variable selection post-hoc on the level of summary statistics. After downloading the PRS from the PGS catalog[55 listed in Suppleplementary Table S10, we imputed PRS into our cohort using our imputed genotypes using the score function of Plink. To account for population structure, we limited our analysis to individuals who self-identified as white, and passed filtering using manual inspection of principal components (Figure S9).

Ethical approval and patient consent Retrospective data collection and analysis was approved by the UCLA IRB. All research was conducted in accordance with the tenets set forth in the Declaration of Helsinki.

Data availability The MRS weights for outcomes that were significantly imputed are located at: https://github.com/cozygene/EHR_MRS_UCLA. The raw UCLA datasets generated during and/or analyzed during the current study are not publicly available due to institutional restrictions on data sharing and privacy concerns.

\section{Discussion}

In this study, we provide a comprehensive investigation of the utility of methylation risk scores in a clinical setting. We used (to our knowledge) the largest methylation biobank cohort produced to date, which includes methylation, genotype, and comprehensive EHR data for all patients. We find that the MRS improved imputation performance over a baseline model by $10.65 \%, 156.31 \%$, and $14.59 \%$ when predicting medication usage, lab panel values, and diagnosis codes respectively. These contributions are significantly more substantial than those obtained by PRS.

The vision of genomic biobanks is that the genomic data will be translated into improved clinical diagnosis and treatment decisions $12,13,79$. In practice, clinical decisions are not expected to be based solely on genomic information, but rather on the combination of the genomic, medical, and demographic information of the patient. While previous studies have used a limited number of key features as a baseline for imputation of a phenotype (e.g., age, sex, and major comorbidities) 50,80 82, to the best of our knowledge, these studies did not take into account the entire familial-genetic or environmental history of the patients. Thus, the question of whether genomic data (methylation or genetics) can be used to improve imputation over the EHR data is critical in order to claim clinical relevance. Our results demonstrate that adding MRS to existing EHR-based imputation frameworks improve imputation accuracy by over $29 \%$ in a clinical context.

It is well appreciated that PRS are sensitive to the studied population, and it is often the case that a PRS developed for one ethnic group performs poorly on others [57, 59]. It is therefore important to evaluate the population effect on MRS performance. For this reason, we measured the transferability 
of our results across different populations, and we observe that the accuracy of the MRS was robust to population structure. This is likely driven by the diversity of the training cohort used, but also due to the fact the we are under-powered to discover subtle differences in imputation accuracy due to our sample sizes. Nonetheless, since we observed very few large differences in accuracy across populations, we are hopeful that our results will inspire future investigations to continue to recruit diverse cohorts and to examine these differences at length with greater sample sizes.

While our study was focused on methylation, there are many other possibilities for the introduction of genomic data in clinical settings. First and foremost, genetic data has been heavily studied by others and large biobanks including genetic data of patients already exists. However, other measurements such as RNA, microbiome, metabolomics, or proteomics may also be relevant. Some of these have logistic and cost considerations at scale. One of the advantages of methylation is that DNA biobanks already exist in large numbers, and the cost of measuring methylation is close to that of measuring genetic data. Moreover, different genomic measurements may provide different snapshots of the patient's data, risk, or health status. Methylation, for example, is known to capture one's smoking status[26, and may therefore be particularly useful for cases in which researchers intend to use self-reported features that may suffer from patient recall bias or honesty. Tangentially, while polygenic risk scores provide a lifetime risk for a patient, methylation risk scores may provide the current risk of the patient over the last few months 83 85], and other genomic information may provide risk with the resolution of days or hours (e.g., RNA or certain metabolomics 86 89]). Nonetheless, owing to the dynamic nature of methylation, it is currently unclear what the range or duration of the methylation risk score are. Furthermore, while methylation patterns are associated with outcomes, it is generally unknown if they cause a disease or are a response to a disease 90 .

To assist the research community in investigating methylation in the context of disease, we provide the MRS predictors for all significantly predicted outcomes at/https://github.com/cozygene/EHR_MRS_UCLA. While our samples were ascertained on kidney and heart disease, we show that our weights successfully replicated across three internal datasets, including studies of Rheumatoid Arthritis and Schizophrenia. Consequently, our weights may be used by researchers and clinicians in different ways. For example, in many epigenome-wide association studies (EWAS), in which associations between specific methylation CpG sites and a phenotype are studied, one may wish to account for patients' comorbidities and medications, which are often not available to the study. Using the MRS database, the researchers leveraging EWAS will be able to incorporate such covariates into their model.

There are multiple potential next steps for the examination of methylation in clinical contexts. First, in this work we focused our attention on the imputation of the phenotypes, or in other words, the inference as to whether the patient is currently diagnosed with a disease. We hope that our findings will be able to be translated to the inference of future clinical events, i.e., prediction of future deterioration or disease occurrence. Second, our analyses did not focus on generating models for a specific patient demographic (e.g. only senior patients) and we were limited to methylation collected from blood samples. As methylation is known to vary across age and tissue type, models may be improved by focusing on individuals of a specific demographic, or by assaying a tissue relevant for a given phenotype (e.g. liver tissue for metabolic disorders. Third, although our evaluation is across the largest dataset which includes both EHR, methylation, and genotype data, the sample size of our study is still moderate compared to genetic studies that are performed on biobanks. Indeed, we demonstrate that for some of the phenotypes, an increase in sample size will likely lead to a substantially improved imputation accuracy (Figure 3 ). Moreover, larger sample size data may be able to reveal the quantity or contribution of genetics verses methylation to the MRS imputation accuracy [49]. In light of our results, as well as the fact that many biobanks have already obtained blood or DNA samples, we recommend that future biobanks consider measuring methylation in addition to the genotypes across a large number of patients. 
Competing Interests I.H. is the president of Clarity Healthcare Analytics Inc, a company that assists hospitals with extracting and using data from their electronic medical records. The company currently owns the rights to the PDW software that was used to extract data from the electronic health record. I.H. receives research funding from Merck Pharmaceuticals. M.C. is a consultant for Edwards Lifesciences (Irvine, CA) and Masimo Corp (Irvine, CA), and has funded research from Edwards Lifesciences and Masimo Corp. He is also the founder of Sironis and he owns patents and receives royalties for closed loop hemodynamic management technologies that have been licensed to Edwards Lifesciences. E.H. is senior vice president of $\mathrm{AI} / \mathrm{ML}$ at OptumLabs (Minnetonka, MN). The other authors declare no competing interests concerning this article.

Author Contribution IPH, E.H., N.Z., B.H., and M.T. conceived of study. M.T. and B.H. performed data analysis under significant contribution and input from S.S., N.R., J.N.C., M.C. and I.H. IPH, N.R., E.H., M.C., I.H., J.N.C. contributed to data acquisition and design. All authors contributed to manuscript writing. 


\section{References}

[1] Cathie Sudlow, John Gallacher, et al. "UK Biobank: An Open Access Resource for Identifying the Causes of a Wide Range of Complex Diseases of Middle and Old Age". In: PLOS Medicine 12.3 (Mar. 2015), e1001779-.

[2] Catherine A McCarty, Russell A Wilke, et al. "Marshfield Clinic Personalized Medicine Research Project (PMRP): design, methods and recruitment for a large population-based biobank". In: Personalized Medicine 2.1 (2005), pp. 49-79.

[3] D M Roden, J M Pulley, et al. "Development of a large-scale de-identified DNA biobank to enable personalized medicine." In: Clin Pharmacol Ther 84.3 (2008), pp. 362-369.

[4] Lisa Bastarache, Jacob J. Hughey, et al. "Phenotype risk scores identify patients with unrecognized Mendelian disease patterns". In: Science 359.6381 (2018), pp. 1233-1239.

[5] Tim Hulsen, Saumya S. Jamuar, et al. "From Big Data to Precision Medicine". In: Frontiers in Medicine 6 (2019), p. 34.

[6] Huiying Liang, Brian Y. Tsui, et al. "Evaluation and accurate diagnoses of pediatric diseases using artificial intelligence". In: Nature Medicine 25.3 (2019), pp. 433-438.

[7] Michelle M. Clark, Amber Hildreth, et al. "Diagnosis of genetic diseases in seriously ill children by rapid whole-genome sequencing and automated phenotyping and interpretation". In: Science Translational Medicine 11.489 (2019).

[8] Kristin M Corey, Sehj Kashyap, et al. "Development and validation of machine learning models to identify high-risk surgical patients using automatically curated electronic health record data (Pythia): A retrospective, single-site study". In: PLOS Medicine 15.11 (Nov. 2018), e1002701e1002701. URL: https://doi.org/10.1371/journal.pmed.1002701.

[9] Brian L. Hill, Robert Brown, et al. "An automated machine learning-based model predicts postoperative mortality using readily-extractable preoperative electronic health record data". English. In: British Journal of Anaesthesia 123.6 (Dec. 2019). Publisher: Elsevier, pp. 877-886. ISSN: 0007-0912, 1471-6771. DOI: $10.1016 / \mathrm{j} \cdot \mathrm{bja} .2019 .07 .030$. URL: https://bjanaesthesia.org/article/ S0007-0912(19) 30646-4/abstract (visited on 05/26/2021).

[10] Amit V Khera, Mark Chaffin, et al. "Genome-wide polygenic scores for common diseases identify individuals with risk equivalent to monogenic mutations." In: Nat Genet 50.9 (2018), pp. 12191224.

[11] Nasim Mavaddat, Kyriaki Michailidou, et al. "Polygenic Risk Scores for Prediction of Breast Cancer and Breast Cancer Subtypes." In: Am J Hum Genet 104.1 (2019), pp. 21-34.

[12] Cathryn M Lewis and Saskia P Hagenaars. "Progressing Polygenic Medicine in Psychiatry Through Electronic Health Records." In: JAMA Psychiatry 76.5 (2019), pp. 470-472.

[13] Cathryn M. Lewis and Evangelos Vassos. "Polygenic risk scores: from research tools to clinical instruments". In: Genome Medicine 12.1 (2020), p. 44.

[14] Miklos D. Kertai, Jonathan D. Mosley, et al. "Predictive Accuracy of a Polygenic Risk Score for Postoperative Atrial Fibrillation After Cardiac Surgery". In: Circulation: Genomic and Precision Medicine 14.2 (2021), e003269.

[15] Feras Hatib, Zhongping Jian, et al. "Machine-learning Algorithm to Predict Hypotension Based on High-fidelity Arterial Pressure Waveform Analysis". In: Anesthesiology 129.4 (Oct. 2018), pp. 663674. URL: http://dx.doi.org/10.1097/ALN.0000000000002300. 
[16] Marije Wijnberge, Bart F. Geerts, et al. "Effect of a Machine Learning-Derived Early Warning System for Intraoperative Hypotension vs Standard Care on Depth and Duration of Intraoperative Hypotension During Elective Noncardiac Surgery: The HYPE Randomized Clinical Trial". en. In: JAMA (Feb. 2020). DOI: 10.1001/jama.2020.0592. URL: https://jamanetwork.com/journals/ $\mathrm{jama/fullarticle/2761469}$ (visited on 02/19/2020).

[17] Varun Gulshan, Lily Peng, et al. "Development and Validation of a Deep Learning Algorithm for Detection of Diabetic Retinopathy in Retinal Fundus Photographs". en. In: JAMA 316.22 (Dec. 2016), pp. 2402-2410. ISSN: 0098-7484. DOI: $10.1001 /$ jama. 2016 . 17216. URL: https : //jamanetwork.com/journals/jama/fullarticle/2588763 (visited on 05/20/2019).

[18] Emma Beede, Elizabeth Baylor, et al. "A Human-Centered Evaluation of a Deep Learning System Deployed in Clinics for the Detection of Diabetic Retinopathy". In: Proceedings of the 2020 CHI Conference on Human Factors in Computing Systems. CHI '20. New York, NY, USA: Association for Computing Machinery, Apr. 2020, pp. 1-12. ISBN: 978-1-4503-6708-0. DOI: 10.1145/3313831. 3376718. URL: https://doi.org/10.1145/3313831.3376718 (visited on 05/25/2021).

[19] Amirata Ghorbani, David Ouyang, et al. "Deep learning interpretation of echocardiograms". en. In: npj Digital Medicine 3.1 (Jan. 2020). Number: 1 Publisher: Nature Publishing Group, pp. 1-10. ISSN: 2398-6352. DOI: 10.1038/s41746-019-0216-8. URL: https://www . nature.com/articles/ s41746-019-0216-8 (visited on 05/14/2021).

[20] Antônio H. Ribeiro, Manoel Horta Ribeiro, et al. "Automatic diagnosis of the 12-lead ECG using a deep neural network". en. In: Nature Communications 11.1 (Apr. 2020). Number: 1 Publisher: Nature Publishing Group, p. 1760. ISSN: 2041-1723. DOI: 10.1038/s41467-020-15432-4. URL: https://www. nature.com/articles/s41467-020-15432-4 (visited on 02/25/2021).

[21] Awni Y Hannun, Pranav Rajpurkar, et al. "Cardiologist-level arrhythmia detection and classification in ambulatory electrocardiograms using a deep neural network". In: Nature Medicine 25.1 (2019), pp. 65-69. DOI: 10.1038/s41591-018-0268-3. URL: https://doi.org/10.1038/s41591018-0268-3.

[22] Matthieu Komorowski, Leo A Celi, et al. "The Artificial Intelligence Clinician learns optimal treatment strategies for sepsis in intensive care". In: Nature Medicine (2018). DOI: 10.1038/s41591018-0213-5. URL: https://doi.org/10.1038/s41591-018-0213-5.

[23] Paige Maas, Myrto Barrdahl, et al. "Breast Cancer Risk From Modifiable and Nonmodifiable Risk Factors Among White Women in the United States". In: JAMA Oncology 2.10 (Oct. 2016), pp. 1295-1302.

[24] Fredrick R Schumacher, Ali Amin Al Olama, et al. "Association analyses of more than 140,000 men identify 63 new prostate cancer susceptibility loci." In: Nat Genet 50.7 (2018), pp. 928-936.

[25] Anke Hüls and Darina Czamara. "Methodological challenges in constructing DNA methylation risk scores". In: Epigenetics 15.1-2 (2020), pp. 1-11.

[26] Ken Lee and Zdenka Pausova. "Cigarette smoking and DNA methylation". In: Frontiers in Genetics 4 (2013), p. 132. ISSN: 1664-8021. DOI: 10.3389/fgene. 2013.00132. URL: https://www . frontiersin.org/article/10.3389/fgene.2013.00132

[27] Joshua M Galanter, Christopher R Gignoux, et al. "Differential methylation between ethnic subgroups reflects the effect of genetic ancestry and environmental exposures". In: eLife 6 (2017), e20532.

[28] Elizabeth Hibler, Lei Huang, et al. "Impact of a diet and activity health promotion intervention on regional patterns of DNA methylation". In: Clinical Epigenetics 11.1 (2019), p. 133.

[29] Alexandra J White, Dale P Sandler, et al. "Recreational and household physical activity at different time points and DNA global methylation." In: Eur J Cancer 49.9 (2013), pp. 2199-2206. 
[30] Fang Fang Zhang, Alfredo Morabia, et al. "Dietary Patterns Are Associated with Levels of Global Genomic DNA Methylation in a Cancer-Free Population". In: The Journal of Nutrition 141.6 (Apr. 2011), pp. 1165-1171.

[31] Katherine J Dick, Christopher P Nelson, et al. "DNA methylation and body-mass index: a genomewide analysis". In: The Lancet 383.9933 (2014), pp. 1990-1998.

[32] Victor V Levenson. "DNA methylation as a universal biomarker". In: Expert Review of Molecular Diagnostics 10.4 (May 2010), pp. 481-488.

[33] Katarzyna Kamińska, Ewelina Nalejska, et al. "Prognostic and Predictive Epigenetic Biomarkers in Oncology". In: Molecular Diagnosis 83 Therapy 23.1 (2019), pp. 83-95.

[34] Audrey Y. Chu, Adrienne Tin, et al. "Epigenome-wide association studies identify DNA methylation associated with kidney function". In: Nature Communications 8.1 (2017), p. 1286.

[35] Yun Liu, Martin J Aryee, et al. "Epigenome-wide association data implicate DNA methylation as an intermediary of genetic risk in rheumatoid arthritis". In: Nature Biotechnology 31 (Jan. 2013), $142 \mathrm{EP}-$.

[36] Vardhman K. Rakyan, Huriya Beyan, et al. "Identification of Type 1 Diabetes-Associated DNA Methylation Variable Positions That Precede Disease Diagnosis". In: PLOS Genetics 7.9 (Sept. 2011), pp. 1-9.

[37] Jimmy L Huynh, Paras Garg, et al. "Epigenome-wide differences in pathology-free regions of multiple sclerosis-affected brains". In: Nature Neuroscience 17.1 (2014), pp. 121-130.

[38] Christopher G. Bell, Andrew E. Teschendorff, et al. "Genome-wide DNA methylation analysis for diabetic nephropathy in type 1 diabetes mellitus". In: BMC Medical Genomics 3.1 (Aug. 2010), p. 33. ISSN: 1755-8794. DOI: 10.1186/1755-8794-3-33. URL: https://doi.org/10.1186/17558794-3-33 (visited on 05/04/2021).

[39] Rahul Mazumder, Trevor Hastie, and Robert Tibshirani. "Spectral Regularization Algorithms for Learning Large Incomplete Matrices". In: Journal of machine learning research : JMLR 11 (Mar. 2010), pp. 2287-2322. ISSN: 1532-4435. URL: http://wWw.ncbi.nlm.nih.gov/pmc/articles/ PMC3087301/,

[40] Frank Dudbridge. "Power and Predictive Accuracy of Polygenic Risk Scores". en. In: PLOS Genetics 9.3 (Mar. 2013). Publisher: Public Library of Science, e1003348. ISSN: 1553-7404. DOI: 10.1371/ journal . pgen .1003348, URL: https://journals .plos . org/plosgenetics/article?id=10 . 1371/journal.pgen.1003348 (visited on 05/03/2021).

[41] Florian Privé, Hugues Aschard, et al. "Efficient analysis of large-scale genome-wide data with two R packages: bigstatsr and bigsnpr". In: Bioinformatics 34.16 (Mar. 2018), pp. 2781-2787.

[42] Eugene Andres Houseman, William P. Accomando, et al. "DNA methylation arrays as surrogate measures of cell mixture distribution". en. In: BMC Bioinformatics 13.1 (May 2012), p. 86. ISSN: 1471-2105. DOI: 10.1186/1471-2105-13-86. URL: https://doi.org/10.1186/1471-2105-13-86 (visited on 03/02/2021).

[43] Joshua C. Denny, Marylyn D. Ritchie, et al. "PheWAS: demonstrating the feasibility of a phenomewide scan to discover gene-disease associations". en. In: Bioinformatics 26.9 (May 2010). Publisher: Oxford Academic, pp. 1205-1210. ISSN: 1367-4803. DOI: 10.1093/bioinformatics/btq126. URL: https : //academic . oup . com/bioinformatics/article/26/9/1205/201211 (visited on $11 / 24 / 2020)$.

[44] Joshua C. Denny, Lisa Bastarache, et al. "Systematic comparison of phenome-wide association study of electronic medical record data and genome-wide association study data". en. In: Nature Biotechnology 31.12 (Dec. 2013). Number: 12 Publisher: Nature Publishing Group, pp. 1102-1111. ISSN: 1546-1696. DOI: 10.1038/nbt.2749. URL: https://www . nature.com/articles/nbt.2749 (visited on 11/10/2020). 
[45] Ernest Beutler and Carol West. "Hematologic differences between African-Americans and whites: the roles of iron deficiency and alpha-thalassemia on hemoglobin levels and mean corpuscular volume." In: Blood 106.2 (2005), pp. 740-745.

[46] Eunjung Lim, Jill Miyamura, and John J Chen. "Racial/Ethnic-Specific Reference Intervals for Common Laboratory Tests: A Comparison among Asians, Blacks, Hispanics, and White." In: Hawaii J Med Public Health 74.9 (2015), pp. 302-310.

[47] Steve Horvath. "DNA methylation age of human tissues and cell types". In: Genome Biology 14.10 (2013), p. 3156.

[48] Paula Singmann, Doron Shem-Tov, et al. "Characterization of whole-genome autosomal differences of DNA methylation between men and women". In: Epigenetics 83 Chromatin 8.1 (2015), p. 43. ISSN: 1756-8935. DOI: 10.1186/s13072-015-0035-3 URL: https ://doi.org/10.1186/s13072015-0035-3.

[49] Daniel Trejo Banos, Daniel L. McCartney, et al. "Bayesian reassessment of the epigenetic architecture of complex traits". In: Nature Communications 11.1 (2020), p. 2865.

[50] Brett K. Beaulieu-Jones, Daniel R. Lavage, et al. "Characterizing and Managing Missing Structured Data in Electronic Health Records: Data Analysis". EN. In: JMIR Medical Informatics 6.1 (Feb. 2018). Company: JMIR Medical Informatics Distributor: JMIR Medical Informatics Institution: JMIR Medical Informatics Label: JMIR Medical Informatics Publisher: JMIR Publications Inc., Toronto, Canada, e8960. DOI: 10.2196/medinform.8960. URL: https://medinform.jmir.org/ 2018/1/e11 (visited on 02/10/2021).

[51] Yosuke Tanigawa, Junyang Qian, et al. "Significant Sparse Polygenic Risk Scores across 813 traits in UK Biobank". In: medRxiv (2021).

[52] Dragana Vuckovic, Erik L. Bao, et al. "The Polygenic and Monogenic Basis of Blood Traits and Diseases". In: Cell 182.5 (2020), 1214-1231.e11.

[53] Nasa Sinnott-Armstrong, Yosuke Tanigawa, et al. "Genetics of 35 blood and urine biomarkers in the UK Biobank". In: Nature Genetics 53.2 (2021), pp. 185-194.

[54] Cathie Sudlow, John Gallacher, et al. "UK Biobank: An Open Access Resource for Identifying the Causes of a Wide Range of Complex Diseases of Middle and Old Age". en. In: PLOS Medicine 12.3 (Mar. 2015). Publisher: Public Library of Science, e1001779. ISSN: 1549-1676. DOI: 10.1371/ journal . pmed . 1001779, URL: https://journals . plos .org/plosmedicine/article? id=10 . 1371/journal.pmed.1001779 (visited on 04/30/2021).

[55] Samuel A. Lambert, Laurent Gil, et al. "The Polygenic Score Catalog as an open database for reproducibility and systematic evaluation". In: Nature Genetics 53.4 (2021), pp. 420-425.

[56] Shaun Purcell, Benjamin Neale, et al. "PLINK: A Tool Set for Whole-Genome Association and Population-Based Linkage Analyses". In: American Journal of Human Genetics 81.3 (Sept. 2007), pp. 559-575. ISSN: 0002-9297. URL: https://www.ncbi.nlm.nih.gov/pmc/articles/PMC1950838/ (visited on 03/15/2021).

[57] L. Duncan, H. Shen, et al. "Analysis of polygenic risk score usage and performance in diverse human populations". en. In: Nature Communications 10.1 (July 2019). Number: 1 Publisher: Nature Publishing Group, p. 3328. ISSN: 2041-1723. DOI: 10.1038/s41467-019-11112-0. URL: https : //www.nature.com/articles/s41467-019-11112-0 (visited on 05/04/2021).

[58] Sini Kerminen, Alicia R. Martin, et al. "Geographic Variation and Bias in the Polygenic Scores of Complex Diseases and Traits in Finland". en. In: The American Journal of Human Genetics 104.6 (June 2019), pp. 1169-1181. ISSN: 0002-9297. DOI: 10.1016/j . ajhg. 2019.05.001. URL: https: //www.sciencedirect.com/science/article/pii/S0002929719301879 (visited on 05/04/2021). 
[59] Alicia R. Martin, Masahiro Kanai, et al. "Clinical use of current polygenic risk scores may exacerbate health disparities". en. In: Nature Genetics 51.4 (Apr. 2019). Number: 4 Publisher: Nature Publishing Group, pp. 584-591. ISSN: 1546-1718. DOI: 10.1038/s41588-019-0379-x. URL: https: //www.nature.com/articles/s41588-019-0379-x (visited on 05/04/2021).

[60] Elior Rahmani, Liat Shenhav, et al. "Genome-wide methylation data mirror ancestry information". In: Epigenetics $\&$ Chromatin 10.1 (Jan. 2017), p. 1. ISSN: 1756-8935. DOI: 10.1186/s13072-0160108-y. URL: https://doi.org/10.1186/s13072-016-0108-y (visited on 12/04/2020).

[61] Richard T. Barfield, Lynn M. Almli, et al. "Accounting for Population Stratification in DNA Methylation Studies". en. In: Genetic Epidemiology 38.3 (2014). _eprint: https://onlinelibrary.wiley.com/doi/pdf/10.1002/g pp. 231-241. ISSN: 1098-2272. DOI: https : // doi .org/10 .1002/gepi . 21789. URL: https : //onlinelibrary.wiley.com/doi/abs/10.1002/gepi.21789 (visited on 12/16/2020).

[62] Erika L. Moen, Xu Zhang, et al. "Genome-Wide Variation of Cytosine Modifications Between European and African Populations and the Implications for Complex Traits". en. In: Genetics 194.4 (Aug. 2013). Publisher: Genetics Section: Investigations, pp. 987-996. Issn: 0016-6731, 1943-2631. DOI: 10.1534/genetics .113.151381. URL: https ://www . genetics . org/content/194/4/987 (visited on $12 / 16 / 2020$ ).

[63] Christopher G Bell, Andrew E Teschendorff, et al. "Genome-wide DNA methylation analysis for diabetic nephropathy in type 1 diabetes mellitus." In: BMC Med Genomics 3 (2010), p. 33.

[64] Eilis Hannon, Emma Dempster, et al. "An integrated genetic-epigenetic analysis of schizophrenia: evidence for co-localization of genetic associations and differential DNA methylation." In: Genome Biol 17.1 (2016), p. 176.

[65] Yun Liu, Martin J Aryee, et al. "Epigenome-wide association data implicate DNA methylation as an intermediary of genetic risk in rheumatoid arthritis." In: Nat Biotechnol 31.2 (2013), pp. 142147.

[66] Ira S Hofer, Eilon Gabel, et al. "A Systematic Approach to Creation of a Perioperative Data Warehouse". In: Anesthesia \& Analgesia 122.6 (2016). ISSN: 0003-2999. URL: https://journals. Iww. com/anesthesia-analgesia/Fulltext/2016/06000/A_Systematic_Approach_to_Creation_ of_a.25.aspx.

[67] Ruth Johnson, Yi Ding, et al. "Leveraging genomic diversity for discovery in an EHR-linked biobank: the UCLA ATLAS Community Health Initiative". In: medRxiv (2021).

[68] Sharon R. Browning and Brian L. Browning. "Rapid and Accurate Haplotype Phasing and MissingData Inference for Whole-Genome Association Studies By Use of Localized Haplotype Clustering". English. In: The American Journal of Human Genetics 81.5 (Nov. 2007). Publisher: Elsevier, pp. 1084-1097. ISSN: 0002-9297, 1537-6605. DOI: 10.1086/521987. URL: https://www.cell.com/ ajhg/abstract/S0002-9297(07)63882-8 (visited on 03/22/2021).

[69] Jian Yang, S. Hong Lee, et al. "GCTA: A Tool for Genome-wide Complex Trait Analysis". In: American Journal of Human Genetics 88.1 (Jan. 2011), pp. 76-82. ISSN: 0002-9297. DOI: 10.1016/ j . ajhg . 2010.11.011. URL: https : //www .ncbi.nlm.nih .gov/pmc/articles/PMC3014363/ (visited on 03/22/2021).

[70] Sayantan Das, Lukas Forer, et al. "Next-generation genotype imputation service and methods." In: Nat Genet 48.10 (2016), pp. 1284-1287.

[71] Po-Ru Loh, Petr Danecek, et al. "Reference-based phasing using the Haplotype Reference Consortium panel". In: Nature Genetics 48.11 (2016), pp. 1443-1448.

[72] Daniel Taliun, Daniel N. Harris, et al. "Sequencing of 53,831 diverse genomes from the NHLBI TOPMed Program". In: Nature 590.7845 (2021), pp. 290-299.

[73] Christian Fuchsberger, Gonçalo R Abecasis, and David A Hinds. "minimac2: faster genotype imputation." In: Bioinformatics 31.5 (2015), pp. 782-784. 
[74] Zongli Xu, Liang Niu, et al. "ENmix: a novel background correction method for Illumina HumanMethylation450 BeadChip". In: Nucleic Acids Research 44.3 (Feb. 2016), e20. IsSN: 0305-1048. DOI: 10.1093/nar/gkv907, URL: https : //www .ncbi.nlm .nih.gov/pmc/articles / PMC4756845/ (visited on $03 / 22 / 2021$ ).

[75] Alex Rubinsteyn, Sergey Feldman, et al. "hammerlab/fancyimpute: Version 0.2.0". In: (Sept. 2017). DOI: 10 .5281/ZENODO . 886614. URL: https : / / zenodo . org/record/886614 \#.WtfmOC-ZNTY (visited on $04 / 18 / 2018$ ).

[76] Gad Abraham, Adam Kowalczyk, et al. "SparSNP: fast and memory-efficient analysis of all SNPs for phenotype prediction." In: BMC Bioinformatics 13 (2012), p. 88.

[77] Bjarni J Vilhjálmsson, Jian Yang, et al. "Modeling Linkage Disequilibrium Increases Accuracy of Polygenic Risk Scores". In: American journal of human genetics 97.4 (Oct. 2015), pp. 576-592.

[78] Tian Ge, Chia-Yen Chen, et al. "Polygenic prediction via Bayesian regression and continuous shrinkage priors". In: Nature Communications 10.1 (2019), p. 1776.

[79] Daniel W Belsky. "Translating Polygenic Analysis for Prevention: From Who to How". In: Circulation. Cardiovascular genetics 10.3 (June 2017), e001798.

[80] Ali Jazayeri, Ou Stella Liang, and Christopher C. Yang. "Imputation of Missing Data in Electronic Health Records Based on Patients'Similarities". In: Journal of Healthcare Informatics Research 4.3 (2020), pp. 295-307.

[81] Riccardo Miotto, Li Li, et al. "Deep Patient: An Unsupervised Representation to Predict the Future of Patients from the Electronic Health Records". In: Scientific Reports 6.1 (2016), p. 26094.

[82] Tao Zheng, Wei Xie, et al. "A machine learning-based framework to identify type 2 diabetes through electronic health records". In: International Journal of Medical Informatics 97 (2017), pp. 120-127.

[83] Elior Rahmani, Noah Zaitlen, et al. "Sparse PCA corrects for cell type heterogeneity in epigenomewide association studies". In: Nature Methods 13 (Mar. 2016). Publisher: Nature Publishing Group, a division of Macmillan Publishers Limited. All Rights Reserved., p. 443. URL: http://dx.doi. org/10.1038/nmeth.3809.

[84] Steve Horvath. "DNA methylation age of human tissues and cell types". In: Genome Biology 14.10 (Dec. 2013), p. 3156. ISSN: 1474-760X. DOI: 10.1186/gb-2013-14-10-r115. URL: https://doi . org/10.1186/gb-2013-14-10-r115 (visited on 05/03/2021).

[85] Kara N Fitzgerald, Romilly Hodges, et al. "Potential reversal of epigenetic age using a diet and lifestyle intervention: a pilot randomized clinical trial". In: Aging 13.7 (Apr. 2021), pp. 9419-9432.

[86] Jiajia Li, Gregory R. Grant, et al. "Chapter Sixteen - Considerations for RNA-seq Analysis of Circadian Rhythms". In: Circadian Rhythms and Biological Clocks, Part A. Ed. by Amita Sehgal. Vol. 551. Methods in Enzymology. Academic Press, 2015, pp. 349-367.

[87] Alexessander Couto Alves, Craig A. Glastonbury, et al. "Fasting and time of day independently modulate circadian rhythm relevant gene expression in adipose and skin tissue". In: BMC Genomics 19.1 (2018), p. 659.

[88] Romanas Chaleckis, Itsuo Murakami, et al. "Individual variability in human blood metabolites identifies age-related differences". In: Proceedings of the National Academy of Sciences 113.16 (2016), pp. 4252-4259.

[89] Gad Asher and Paolo Sassone-Corsi. "Time for food: the intimate interplay between nutrition, metabolism, and the circadian clock." In: Cell 161.1 (2015), pp. 84-92.

[90] Caroline L Relton and George Davey Smith. "Two-step epigenetic Mendelian randomization: a strategy for establishing the causal role of epigenetic processes in pathways to disease." In: Int $J$ Epidemiol 41.1 (2012), pp. 161-176. 


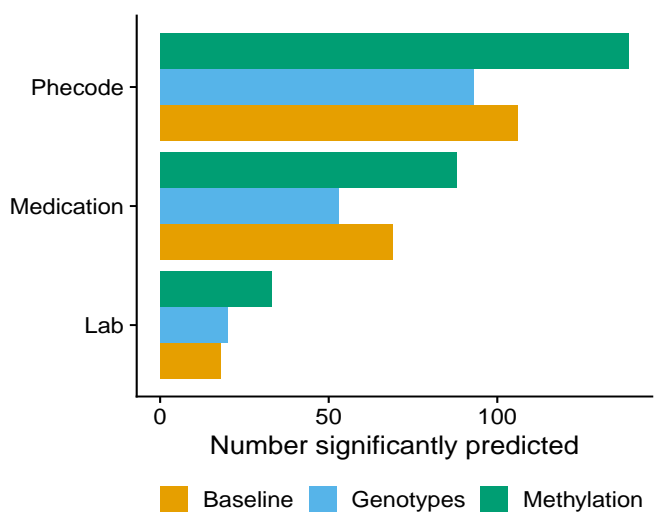

Figure S1. Significantly predicted outcomes per data type. Total number of significantly predicted outcomes when using the baseline alone, as well as including either set of genomic features in addition to the baseline. We used an association test of the cross-validated predictors and the true outcome and adjusted for multiple testing using Bonferroni correction at a nominal threshold of 0.05 .



Figure S2. Significantly predicted medications prescribed within one year of collection date or EHR history. Total number of significantly predicted medications when using the baseline alone, as well as including either set of genomic features in addition to the baseline. We used an association test of the cross-validated predictors and the true outcome and adjusted for multiple testing using Bonferroni correction at a nominal threshold of 0.05. Using a patient's entire EHR history to generate their list of medications resulted in more significant associations than using just the year prior to sample collection date. 


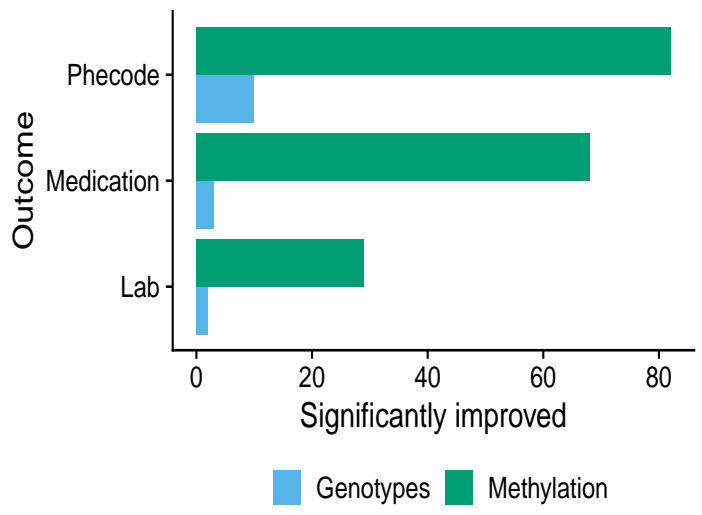

Figure S3. Number of outcomes significantly improved when adding the corresponding predictor. Similarly to the tests in which we surveyed whether the MRS or PRS predictors added predictive power over the baseline predictor, we conducted an analysis in which we examined whether the MRS predictor adds predictive power over the PRS predictor (Green "Methylation"), and whether the PRS predictor adds predictive power over the MRS predictor (Blue "Genotypes"). The MRS predictor improved the performance of the PRS predictor for a substantial number of outcomes

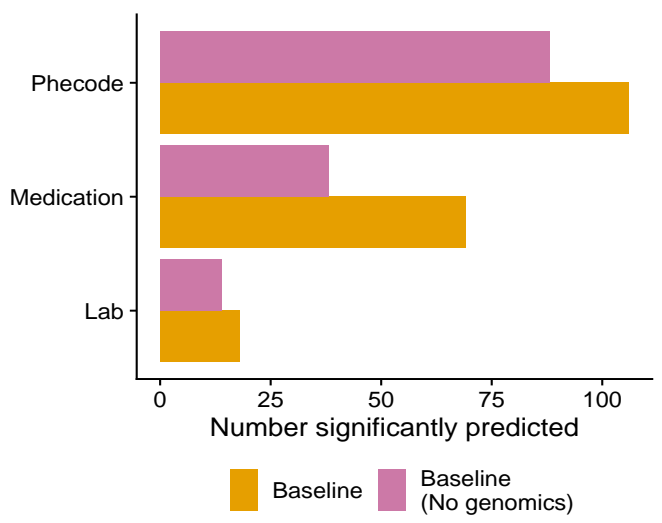

Figure S4. Significantly predicted outcomes across baseline models. Total number of significantly predicted outcomes when using the baseline model with and without variables derived from genomics (ancestry principal components, methylation-estimated cell-type composition estimates). We used an association test of the cross-validated predictors and the true outcome and adjusted for multiple testing using Bonferroni correction at a nominal threshold of 0.05. 
medRxiv preprint doi: https://doi.org/10.1101/2022.02.07.22270047; this version posted February 9, 2022. The copyright holder for this preprint (which was not certified by peer review) is the author/funder, who has granted medRxiv a license to display the preprint in perpetuity.

\section{It is made available under a CC-BY-NC-ND 4.0 International license.}
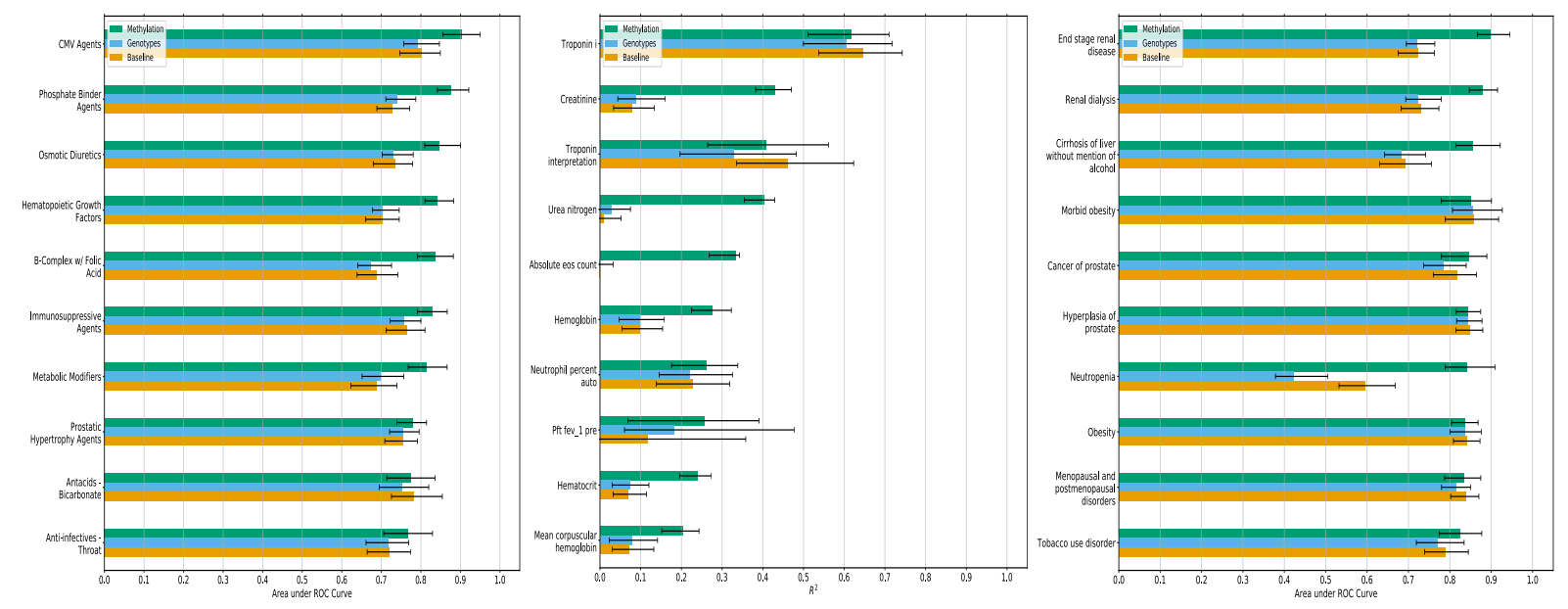

Figure S5. Significantly-predicted outcomes per data type The top 10 methylation-predicted (Left) medications, (Middle) labs, and (Right) Phecodes, with Baseline and Genotype prediction performance results for comparison. 


\begin{tabular}{|c|c|c|c|}
\hline & & Missing & Overall \\
\hline $\mathrm{n}$ & & & 831 \\
\hline Age, mean (SD) & & 0 & $61.0(15.8)$ \\
\hline \multirow[t]{2}{*}{ Sex, n $(\%)$} & $\mathrm{F}$ & 0 & $352(42.4)$ \\
\hline & M & & $479(57.6)$ \\
\hline BMI, mean (SD) & & 1 & $27.2(6.6)$ \\
\hline \multirow[t]{4}{*}{ AKIN Classification, n (\%) } & 0.0 & 0 & $537(64.6)$ \\
\hline & 1.0 & & $189(22.7)$ \\
\hline & 2.0 & & $27(3.2)$ \\
\hline & 3.0 & & $78(9.4)$ \\
\hline \multirow[t]{2}{*}{ GFR $>38, \mathrm{n}(\%)$} & False & 0 & $375(45.1)$ \\
\hline & True & & $456(54.9)$ \\
\hline \multirow[t]{2}{*}{ Heart Attack, n (\%) } & False & 601 & $146(63.5)$ \\
\hline & True & & $84(36.5)$ \\
\hline \multirow[t]{7}{*}{ Self-Reported Ethnicity, n (\%) } & Cuban & 0 & $2(0.2)$ \\
\hline & Hispanic or Latino & & $116(14.0)$ \\
\hline & Hispanic/Spanish origin Other & & $14(1.7)$ \\
\hline & Mexican, Mexican American, Chicano/a & & $37(4.5)$ \\
\hline & Not Hispanic or Latino & & $655(78.8)$ \\
\hline & Patient Refused & & $5(0.6)$ \\
\hline & Puerto Rican & & $2(0.2)$ \\
\hline \multirow[t]{8}{*}{ Self-Reported Race, n (\%) } & American Indian & 0 & $2(0.2)$ \\
\hline & Asian & & $73(8.8)$ \\
\hline & Black & & $72(8.7)$ \\
\hline & Declined to Specify & & $6(0.7)$ \\
\hline & Other Race & & $132(15.9)$ \\
\hline & Pacific Islander & & $3(0.4)$ \\
\hline & Unknown & & $1(0.1)$ \\
\hline & White or Caucasian & & $542(65.2)$ \\
\hline
\end{tabular}

Table S1. Cohort patient demographics. AKIN is the Acute Kidney Injury Network Classification, BMI is Body Mass Index, GFR is glomerular filtration rate. 
Table S2. Mean (95\% confidence interval) area under the ROC curve for predicting medication usage, grouped by pharmaceutical subclass, using the baseline, methylation data, and genotype data. Confidence intervals determined using bootstrapping.

\begin{tabular}{|c|c|c|c|}
\hline Pharmaceutical Subclass & Baseline & Methylation & Genotypes \\
\hline CMV Agents & $0.80(0.75-0.85)$ & $0.90(0.86-0.94)$ & $0.79(0.74-0.84)$ \\
\hline Phosphate Binder Agents & $0.73(0.68-0.77)$ & $0.88(0.84-0.91)$ & $0.74(0.70-0.78)$ \\
\hline Osmotic Diuretics & $0.74(0.69-0.78)$ & $0.85(0.81-0.88)$ & $0.73(0.68-0.78)$ \\
\hline Hematopoietic Growth Factors & $0.70(0.66-0.75)$ & $0.84(0.81-0.87)$ & $0.70(0.66-0.75)$ \\
\hline B-Complex w/ Folic Acid & $0.69(0.64-0.74)$ & $0.84(0.79-0.87)$ & $0.67(0.62-0.72)$ \\
\hline Immunosuppressive Agents & $0.77(0.72-0.81)$ & $0.83(0.79-0.86)$ & $0.76(0.70-0.80)$ \\
\hline Metabolic Modifiers & $0.69(0.63-0.74)$ & $0.81(0.77-0.86)$ & $0.70(0.63-0.75)$ \\
\hline Prostatic Hypertrophy Agents & $0.76(0.72-0.79)$ & $0.78(0.74-0.81)$ & $0.76(0.71-0.79)$ \\
\hline Antacids - Bicarbonate & $0.78(0.71-0.85)$ & $0.78(0.71-0.83)$ & $0.75(0.69-0.81)$ \\
\hline Anti-infectives - Throat & $0.72(0.67-0.77)$ & $0.77(0.71-0.82)$ & $0.72(0.66-0.78)$ \\
\hline Cycloplegic Mydriatics & $0.76(0.71-0.82)$ & $0.75(0.68-0.81)$ & $0.76(0.69-0.82)$ \\
\hline Thrombolytic Enzymes & $0.67(0.60-0.74)$ & $0.75(0.68-0.81)$ & $0.63(0.56-0.70)$ \\
\hline Plasma Proteins & $0.67(0.62-0.72)$ & $0.74(0.69-0.78)$ & $0.66(0.61-0.71)$ \\
\hline Potassium Removing Agents & $0.64(0.59-0.70)$ & $0.74(0.69-0.79)$ & $0.62(0.56-0.67)$ \\
\hline Cephalosporins - 4th Generation & $0.67(0.59-0.74)$ & $0.73(0.64-0.80)$ & $0.65(0.57-0.74)$ \\
\hline Gallstone Solubilizing Agents & $0.59(0.50-0.69)$ & $0.72(0.64-0.79)$ & $0.50(0.41-0.59)$ \\
\hline Imidazole-Related Antifungals & $0.69(0.64-0.73)$ & $0.72(0.67-0.77)$ & $0.68(0.63-0.73)$ \\
\hline HMG CoA Reductase Inhibitors & $0.72(0.67-0.75)$ & $0.72(0.68-0.75)$ & $0.71(0.68-0.74)$ \\
\hline Alkalinizers & $0.70(0.62-0.76)$ & $0.71(0.63-0.78)$ & $0.68(0.59-0.75)$ \\
\hline Bone Density Regulators & $0.70(0.63-0.75)$ & $0.71(0.65-0.75)$ & $0.70(0.64-0.76)$ \\
\hline Parenteral Therapy Supplies & $0.52(0.45-0.59)$ & $0.71(0.65-0.76)$ & $0.60(0.54-0.66)$ \\
\hline Vasodilators & $0.54(0.49-0.59)$ & $0.70(0.66-0.75)$ & $0.48(0.43-0.52)$ \\
\hline Salicylates & $0.69(0.65-0.72)$ & $0.70(0.66-0.73)$ & $0.68(0.64-0.72)$ \\
\hline Ophthalmic Local Anesthetics & $0.69(0.63-0.76)$ & $0.69(0.63-0.76)$ & $0.68(0.61-0.76)$ \\
\hline Proton Pump Inhibitors & $0.62(0.58-0.66)$ & $0.69(0.66-0.73)$ & $0.61(0.57-0.66)$ \\
\hline Impotence Agents & $0.70(0.64-0.75)$ & $0.68(0.62-0.74)$ & $0.67(0.60-0.73)$ \\
\hline Ophthalmic Steroids & $0.70(0.64-0.75)$ & $0.68(0.62-0.73)$ & $0.68(0.62-0.74)$ \\
\hline Diabetic Supplies & $0.64(0.58-0.69)$ & $0.67(0.62-0.72)$ & $0.62(0.56-0.67)$ \\
\hline Phosphate & $0.65(0.57-0.72)$ & $0.67(0.60-0.74)$ & $0.60(0.52-0.66)$ \\
\hline Loop Diuretics & $0.63(0.60-0.67)$ & $0.67(0.63-0.71)$ & $0.63(0.59-0.67)$ \\
\hline Antiseptics - Mouth/Throat & $0.63(0.57-0.68)$ & $0.67(0.62-0.73)$ & $0.62(0.54-0.67)$ \\
\hline Anti-infective Agents - Misc. & $0.61(0.56-0.65)$ & $0.67(0.62-0.72)$ & $0.60(0.55-0.65)$ \\
\hline Specialty Vitamins Products & $0.60(0.52-0.68)$ & $0.67(0.58-0.75)$ & $0.61(0.52-0.69)$ \\
\hline Anti-infective Misc. - Combinations & $0.64(0.59-0.68)$ & $0.67(0.62-0.72)$ & $0.62(0.57-0.66)$ \\
\hline Iron & $0.66(0.62-0.71)$ & $0.67(0.62-0.71)$ & $0.66(0.61-0.71)$ \\
\hline Cephalosporins - 3rd Generation & $0.62(0.57-0.66)$ & $0.66(0.63-0.71)$ & $0.61(0.57-0.65)$ \\
\hline Glucocorticosteroids & $0.59(0.55-0.63)$ & $0.66(0.63-0.70)$ & $0.58(0.54-0.62)$ \\
\hline Antihistamines - Ethanolamines & $0.62(0.58-0.65)$ & $0.66(0.62-0.70)$ & $0.62(0.58-0.66)$ \\
\hline Fluoroquinolones & $0.54(0.50-0.58)$ & $0.66(0.62-0.70)$ & $0.52(0.48-0.57)$ \\
\hline Calcium & $0.61(0.57-0.65)$ & $0.66(0.62-0.71)$ & $0.60(0.56-0.64)$ \\
\hline Benzodiazepines & $0.61(0.57-0.66)$ & $0.66(0.62-0.70)$ & $0.61(0.56-0.64)$ \\
\hline Biguanides & $0.67(0.62-0.71)$ & $0.66(0.61-0.71)$ & $0.65(0.60-0.70)$ \\
\hline Local Anesthetic Combinations & $0.57(0.53-0.62)$ & $0.66(0.61-0.70)$ & $0.56(0.52-0.61)$ \\
\hline
\end{tabular}


Table S2. Mean (95\% confidence interval) area under the ROC curve for predicting medication usage, grouped by pharmaceutical subclass, using the baseline, methylation data, and genotype data. Confidence intervals determined using bootstrapping.

\begin{tabular}{|c|c|c|c|}
\hline Pharmaceutical Subclass & Baseline & Methylation & Genotypes \\
\hline Ophthalmics - Misc. & $0.66(0.60-0.72)$ & $0.66(0.60-0.71)$ & $0.65(0.60-0.70)$ \\
\hline Antacid Combinations & $0.63(0.58-0.68)$ & $0.66(0.61-0.70)$ & $0.62(0.56-0.67)$ \\
\hline Dibenzapines & $0.62(0.56-0.68)$ & $0.66(0.58-0.72)$ & $0.58(0.52-0.63)$ \\
\hline Nitrates & $0.64(0.61-0.68)$ & $0.66(0.61-0.70)$ & $0.63(0.59-0.67)$ \\
\hline Insulin & $0.63(0.58-0.67)$ & $0.66(0.61-0.69)$ & $0.62(0.57-0.66)$ \\
\hline Liquid Vehicles & $0.59(0.51-0.67)$ & $0.65(0.58-0.73)$ & $0.58(0.51-0.66)$ \\
\hline Alternative Medicine - M's & $0.55(0.49-0.61)$ & $0.65(0.59-0.71)$ & $0.59(0.52-0.64)$ \\
\hline 5-HT3 Receptor Antagonists & $0.57(0.53-0.60)$ & $0.65(0.61-0.69)$ & $0.55(0.51-0.60)$ \\
\hline Antiperistaltic Agents & $0.55(0.48-0.62)$ & $0.65(0.57-0.73)$ & $0.51(0.44-0.58)$ \\
\hline Analgesics Other & $0.59(0.55-0.63)$ & $0.65(0.61-0.68)$ & $0.58(0.54-0.62)$ \\
\hline Carbohydrates & $0.61(0.57-0.65)$ & $0.65(0.61-0.69)$ & $0.59(0.54-0.64)$ \\
\hline Laxatives - Miscellaneous & $0.61(0.57-0.65)$ & $0.65(0.61-0.68)$ & $0.59(0.55-0.64)$ \\
\hline Alpha-Beta Blockers & $0.57(0.52-0.61)$ & $0.65(0.60-0.69)$ & $0.57(0.53-0.61)$ \\
\hline Saline Laxatives & $0.59(0.54-0.63)$ & $0.64(0.60-0.70)$ & $0.58(0.54-0.62)$ \\
\hline Potassium & $0.59(0.55-0.63)$ & $0.64(0.60-0.68)$ & $0.58(0.54-0.62)$ \\
\hline Stimulant Laxatives & $0.60(0.56-0.64)$ & $0.64(0.60-0.68)$ & $0.60(0.56-0.64)$ \\
\hline Urinary Anti-infectives & $0.60(0.53-0.67)$ & $0.64(0.55-0.71)$ & $0.51(0.42-0.58)$ \\
\hline Calcium Channel Blockers & $0.59(0.56-0.63)$ & $0.64(0.60-0.67)$ & $0.60(0.56-0.63)$ \\
\hline Glycopeptides & $0.60(0.56-0.65)$ & $0.64(0.59-0.68)$ & $0.59(0.54-0.63)$ \\
\hline Magnesium & $0.58(0.54-0.62)$ & $0.64(0.60-0.67)$ & $0.57(0.53-0.61)$ \\
\hline Heparins And Heparinoid-Like Agents & $0.62(0.58-0.65)$ & $0.64(0.60-0.67)$ & $0.61(0.57-0.64)$ \\
\hline Bicarbonates & $0.60(0.53-0.67)$ & $0.63(0.56-0.70)$ & $0.61(0.53-0.68)$ \\
\hline Electrolyte Mixtures & $0.58(0.55-0.62)$ & $0.63(0.60-0.67)$ & $0.57(0.54-0.61)$ \\
\hline Thyroid Hormones & $0.64(0.58-0.69)$ & $0.63(0.58-0.68)$ & $0.63(0.58-0.68)$ \\
\hline Antihypertensive Combinations & $0.64(0.56-0.70)$ & $0.63(0.57-0.71)$ & $0.59(0.50-0.67)$ \\
\hline Folic Acid/Folates & $0.61(0.54-0.67)$ & $0.63(0.58-0.69)$ & $0.60(0.55-0.66)$ \\
\hline Diagnostic Radiopharmaceuticals & $0.58(0.54-0.62)$ & $0.63(0.60-0.67)$ & $0.57(0.52-0.61)$ \\
\hline Surfactant Laxatives & $0.60(0.57-0.64)$ & $0.63(0.58-0.66)$ & $0.60(0.56-0.64)$ \\
\hline Thiazides and Thiazide-Like Diuretics & $0.65(0.61-0.71)$ & $0.63(0.58-0.68)$ & $0.63(0.58-0.67)$ \\
\hline Expectorants & $0.57(0.47-0.65)$ & $0.63(0.54-0.72)$ & $0.44(0.36-0.52)$ \\
\hline Platelet Aggregation Inhibitors & $0.64(0.59-0.68)$ & $0.63(0.58-0.67)$ & $0.62(0.58-0.67)$ \\
\hline Antiflatulents & $0.56(0.50-0.61)$ & $0.63(0.58-0.68)$ & $0.54(0.48-0.60)$ \\
\hline Vasopressors & $0.57(0.53-0.61)$ & $0.62(0.59-0.65)$ & $0.55(0.52-0.60)$ \\
\hline Opioid Antagonists & $0.60(0.54-0.65)$ & $0.62(0.58-0.67)$ & $0.60(0.54-0.65)$ \\
\hline Antibiotics - Topical & $0.55(0.50-0.60)$ & $0.62(0.58-0.67)$ & $0.50(0.45-0.56)$ \\
\hline Antidiarrheal/Probiotic Agents - Misc. & $0.56(0.49-0.63)$ & $0.62(0.55-0.69)$ & $0.51(0.43-0.59)$ \\
\hline Serotonin-Norepinephrine Reuptake Inhibitors (S... & $0.68(0.61-0.74)$ & $0.62(0.54-0.70)$ & $0.66(0.58-0.73)$ \\
\hline Phenothiazines & $0.52(0.46-0.56)$ & $0.62(0.56-0.66)$ & $0.46(0.41-0.50)$ \\
\hline Beta Blockers Cardio-Selective & $0.54(0.51-0.58)$ & $0.61(0.58-0.65)$ & $0.53(0.49-0.57)$ \\
\hline Misc. Nutritional Substances & $0.55(0.49-0.59)$ & $0.61(0.56-0.67)$ & $0.54(0.49-0.59)$ \\
\hline Alpha-2 Receptor Antagonists (Tetracyclics) & $0.66(0.56-0.75)$ & $0.61(0.51-0.70)$ & $0.67(0.58-0.75)$ \\
\hline Bronchodilators - Anticholinergics & $0.63(0.58-0.67)$ & $0.61(0.56-0.67)$ & $0.61(0.56-0.66)$ \\
\hline Tetracyclines & $0.51(0.45-0.59)$ & $0.61(0.56-0.67)$ & $0.40(0.35-0.46)$ \\
\hline
\end{tabular}


Table S2. Mean (95\% confidence interval) area under the ROC curve for predicting medication usage, grouped by pharmaceutical subclass, using the baseline, methylation data, and genotype data. Confidence intervals determined using bootstrapping.

\begin{tabular}{|c|c|c|c|}
\hline Pharmaceutical Subclass & Baseline & Methylation & Genotypes \\
\hline Antacids - Calcium Salts & $0.58(0.53-0.63)$ & $0.61(0.56-0.66)$ & $0.55(0.48-0.60)$ \\
\hline Penicillin Combinations & $0.57(0.52-0.61)$ & $0.61(0.56-0.65)$ & $0.55(0.51-0.60)$ \\
\hline Gout Agents & $0.65(0.60-0.69)$ & $0.61(0.54-0.66)$ & $0.63(0.57-0.68)$ \\
\hline Radiographic Contrast Media & $0.61(0.57-0.64)$ & $0.61(0.57-0.64)$ & $0.60(0.56-0.63)$ \\
\hline Sodium & $0.47(0.43-0.52)$ & $0.61(0.56-0.65)$ & $0.54(0.48-0.59)$ \\
\hline Diagnostic Tests & $0.60(0.55-0.65)$ & $0.61(0.55-0.66)$ & $0.57(0.52-0.63)$ \\
\hline Sympathomimetics & $0.59(0.55-0.62)$ & $0.61(0.57-0.65)$ & $0.57(0.53-0.61)$ \\
\hline Antiarrhythmics Type III & $0.56(0.48-0.62)$ & $0.60(0.53-0.67)$ & $0.53(0.46-0.61)$ \\
\hline Antihistamines-Topical & $0.48(0.41-0.57)$ & $0.60(0.53-0.68)$ & $0.39(0.31-0.47)$ \\
\hline Antidotes and Specific Antagonists & $0.39(0.33-0.46)$ & $0.60(0.54-0.67)$ & $0.59(0.52-0.65)$ \\
\hline Coumarin Anticoagulants & $0.62(0.57-0.68)$ & $0.60(0.55-0.66)$ & $0.58(0.52-0.62)$ \\
\hline Bacterial Vaccines & $0.56(0.51-0.62)$ & $0.60(0.55-0.65)$ & $0.56(0.52-0.61)$ \\
\hline Genitourinary Irrigants & $0.41(0.36-0.46)$ & $0.60(0.55-0.65)$ & $0.54(0.49-0.59)$ \\
\hline Anesthetics Topical Oral & $0.63(0.57-0.69)$ & $0.60(0.53-0.66)$ & $0.60(0.54-0.67)$ \\
\hline Cobalamins & $0.60(0.54-0.65)$ & $0.60(0.55-0.66)$ & $0.57(0.52-0.63)$ \\
\hline Posterior Pituitary Hormones & $0.56(0.50-0.62)$ & $0.59(0.53-0.66)$ & $0.50(0.43-0.56)$ \\
\hline Gastrointestinal Stimulants & $0.52(0.47-0.57)$ & $0.59(0.55-0.65)$ & $0.53(0.48-0.57)$ \\
\hline Antiadrenergic Antihypertensives & $0.55(0.48-0.60)$ & $0.59(0.54-0.65)$ & $0.49(0.43-0.54)$ \\
\hline Angiotensin II Receptor Antagonists & $0.61(0.56-0.66)$ & $0.59(0.55-0.63)$ & $0.58(0.54-0.62)$ \\
\hline Antitussives & $0.50(0.43-0.56)$ & $0.59(0.53-0.65)$ & $0.50(0.44-0.55)$ \\
\hline B-Complex Vitamins & $0.49(0.41-0.57)$ & $0.59(0.47-0.67)$ & $0.48(0.40-0.56)$ \\
\hline Cephalosporins - 1st Generation & $0.55(0.51-0.59)$ & $0.59(0.55-0.63)$ & $0.53(0.49-0.58)$ \\
\hline Diagnostic Drugs & $0.59(0.54-0.63)$ & $0.59(0.54-0.63)$ & $0.59(0.54-0.63)$ \\
\hline Potassium Sparing Diuretics & $0.60(0.53-0.66)$ & $0.58(0.52-0.66)$ & $0.57(0.50-0.64)$ \\
\hline Selective Serotonin Reuptake Inhibitors (SSRIs) & $0.59(0.55-0.64)$ & $0.58(0.53-0.64)$ & $0.57(0.52-0.62)$ \\
\hline Anesthetics - Misc. & $0.53(0.49-0.56)$ & $0.58(0.55-0.62)$ & $0.52(0.49-0.55)$ \\
\hline Irrigation Solutions & $0.58(0.50-0.64)$ & $0.58(0.51-0.64)$ & $0.55(0.47-0.62)$ \\
\hline Lozenges & $0.58(0.50-0.65)$ & $0.58(0.49-0.66)$ & $0.51(0.43-0.60)$ \\
\hline Aminoglycosides & $0.55(0.48-0.62)$ & $0.58(0.50-0.64)$ & $0.45(0.38-0.54)$ \\
\hline Non-Barbiturate Hypnotics & $0.55(0.51-0.59)$ & $0.57(0.53-0.61)$ & $0.53(0.49-0.57)$ \\
\hline Ophthalmic Anti-infectives & $0.61(0.56-0.66)$ & $0.57(0.53-0.63)$ & $0.61(0.54-0.66)$ \\
\hline Antiarrhythmics Type I-B & $0.54(0.50-0.58)$ & $0.57(0.54-0.62)$ & $0.51(0.48-0.55)$ \\
\hline Oil Soluble Vitamins & $0.57(0.53-0.61)$ & $0.57(0.53-0.61)$ & $0.59(0.56-0.64)$ \\
\hline Direct Factor Xa Inhibitors & $0.58(0.52-0.64)$ & $0.57(0.51-0.63)$ & $0.57(0.51-0.63)$ \\
\hline H-2 Antagonists & $0.52(0.48-0.57)$ & $0.57(0.53-0.62)$ & $0.50(0.47-0.55)$ \\
\hline Multivitamins & $0.51(0.46-0.55)$ & $0.57(0.52-0.62)$ & $0.52(0.47-0.57)$ \\
\hline Hemostatics - Topical & $0.57(0.50-0.63)$ & $0.57(0.50-0.64)$ & $0.53(0.47-0.58)$ \\
\hline Artificial Tears and Lubricants & $0.56(0.48-0.63)$ & $0.57(0.51-0.64)$ & $0.58(0.51-0.65)$ \\
\hline Anti-inflammatory Agents - Topical & $0.58(0.52-0.65)$ & $0.57(0.51-0.64)$ & $0.58(0.51-0.64)$ \\
\hline Opioid Agonists & $0.58(0.53-0.62)$ & $0.57(0.53-0.61)$ & $0.55(0.51-0.60)$ \\
\hline Leukotriene Modulators & $0.55(0.46-0.63)$ & $0.57(0.48-0.66)$ & $0.50(0.40-0.57)$ \\
\hline Antianxiety Agents - Misc. & $0.58(0.52-0.65)$ & $0.57(0.50-0.64)$ & $0.53(0.46-0.60)$ \\
\hline Local Anesthetics - Amides & $0.58(0.53-0.62)$ & $0.57(0.53-0.61)$ & $0.56(0.51-0.60)$ \\
\hline
\end{tabular}


Table S2. Mean (95\% confidence interval) area under the ROC curve for predicting medication usage, grouped by pharmaceutical subclass, using the baseline, methylation data, and genotype data. Confidence intervals determined using bootstrapping.

\begin{tabular}{|c|c|c|c|}
\hline Pharmaceutical Subclass & Baseline & Methylation & Genotypes \\
\hline Water Soluble Vitamins & $0.57(0.52-0.62)$ & $0.57(0.52-0.62)$ & $0.56(0.51-0.61)$ \\
\hline Nondepolarizing Muscle Relaxants & $0.56(0.52-0.60)$ & $0.57(0.52-0.61)$ & $0.54(0.51-0.58)$ \\
\hline Urinary Antispasmodic - Antimuscarinics (Antich... & $0.59(0.51-0.66)$ & $0.57(0.49-0.64)$ & $0.59(0.51-0.66)$ \\
\hline Bulk Laxatives & $0.53(0.46-0.61)$ & $0.56(0.50-0.64)$ & $0.53(0.45-0.61)$ \\
\hline Antiemetics - Anticholinergic & $0.53(0.46-0.60)$ & $0.56(0.50-0.63)$ & $0.45(0.39-0.51)$ \\
\hline Aminopenicillins & $0.55(0.49-0.60)$ & $0.56(0.51-0.62)$ & $0.54(0.48-0.59)$ \\
\hline Serotonin Modulators & $0.53(0.48-0.58)$ & $0.56(0.51-0.61)$ & $0.53(0.48-0.59)$ \\
\hline Viral Vaccines & $0.56(0.51-0.61)$ & $0.56(0.51-0.60)$ & $0.52(0.47-0.57)$ \\
\hline Laxative Combinations & $0.57(0.52-0.63)$ & $0.56(0.50-0.61)$ & $0.55(0.50-0.61)$ \\
\hline Antimyasthenic/Cholinergic Agents & $0.54(0.50-0.59)$ & $0.56(0.50-0.60)$ & $0.52(0.46-0.58)$ \\
\hline Azithromycin & $0.59(0.54-0.63)$ & $0.55(0.51-0.60)$ & $0.57(0.53-0.63)$ \\
\hline Local Anesthetics - Topical & $0.54(0.49-0.58)$ & $0.55(0.51-0.60)$ & $0.52(0.47-0.56)$ \\
\hline ACE Inhibitors & $0.51(0.47-0.55)$ & $0.55(0.50-0.60)$ & $0.48(0.43-0.52)$ \\
\hline Herpes Agents & $0.53(0.45-0.59)$ & $0.55(0.48-0.62)$ & $0.48(0.41-0.55)$ \\
\hline Influenza Agents & $0.51(0.44-0.58)$ & $0.54(0.48-0.61)$ & $0.45(0.38-0.53)$ \\
\hline Beta Blockers Non-Selective & $0.52(0.44-0.59)$ & $0.54(0.45-0.64)$ & $0.59(0.50-0.69)$ \\
\hline Sulfonylureas & $0.59(0.51-0.66)$ & $0.54(0.47-0.62)$ & $0.61(0.55-0.69)$ \\
\hline Nasal Steroids & $0.56(0.51-0.60)$ & $0.54(0.50-0.59)$ & $0.51(0.46-0.57)$ \\
\hline Antispasmodics & $0.56(0.52-0.60)$ & $0.54(0.50-0.58)$ & $0.56(0.52-0.59)$ \\
\hline Cough/Cold/Allergy Combinations & $0.58(0.52-0.65)$ & $0.54(0.48-0.61)$ & $0.55(0.49-0.63)$ \\
\hline Cephalosporins - 2nd Generation & $0.52(0.44-0.59)$ & $0.54(0.47-0.62)$ & $0.49(0.42-0.56)$ \\
\hline Opioid Combinations & $0.53(0.49-0.57)$ & $0.54(0.50-0.58)$ & $0.50(0.46-0.53)$ \\
\hline Anticonvulsants - Misc. & $0.54(0.49-0.58)$ & $0.54(0.50-0.58)$ & $0.51(0.47-0.55)$ \\
\hline Multiple Vitamins w/ Minerals & $0.58(0.51-0.65)$ & $0.54(0.47-0.59)$ & $0.54(0.49-0.60)$ \\
\hline Protamine & $0.53(0.46-0.61)$ & $0.54(0.44-0.64)$ & $0.50(0.39-0.59)$ \\
\hline Lincosamides & $0.52(0.46-0.59)$ & $0.53(0.47-0.59)$ & $0.52(0.44-0.59)$ \\
\hline Hemostatics - Systemic & $0.46(0.37-0.55)$ & $0.53(0.44-0.62)$ & $0.54(0.47-0.62)$ \\
\hline Antihistamines - Non-Sedating & $0.54(0.47-0.60)$ & $0.52(0.46-0.57)$ & $0.53(0.46-0.58)$ \\
\hline Acne Products & $0.59(0.49-0.66)$ & $0.50(0.41-0.60)$ & $0.41(0.33-0.50)$ \\
\hline Miscellaneous Contrast Media & $0.49(0.46-0.53)$ & $0.50(0.46-0.54)$ & $0.53(0.49-0.57)$ \\
\hline Nonsteroidal Anti-inflammatory Agents (NSAIDs) & $0.52(0.49-0.57)$ & $0.49(0.45-0.53)$ & $0.53(0.48-0.57)$ \\
\hline Toxoid Combinations & $0.55(0.49-0.63)$ & $0.49(0.42-0.57)$ & $0.55(0.48-0.63)$ \\
\hline Corticosteroids - Topical & $0.51(0.46-0.56)$ & $0.49(0.43-0.53)$ & $0.43(0.38-0.47)$ \\
\hline Tricyclic Agents & $0.53(0.44-0.61)$ & $0.48(0.39-0.56)$ & $0.58(0.51-0.65)$ \\
\hline Central Muscle Relaxants & $0.52(0.48-0.57)$ & $0.48(0.43-0.52)$ & $0.48(0.43-0.53)$ \\
\hline Depolarizing Muscle Relaxants & $0.53(0.47-0.58)$ & $0.46(0.40-0.51)$ & $0.49(0.43-0.54)$ \\
\hline Antifungals - Topical & $0.50(0.44-0.55)$ & $0.45(0.40-0.50)$ & $0.48(0.43-0.54)$ \\
\hline Cardiac Glycosides & $0.52(0.42-0.60)$ & $0.43(0.33-0.52)$ & $0.54(0.46-0.63)$ \\
\hline Alternative Medicine - C's & $0.53(0.47-0.60)$ & $0.37(0.30-0.44)$ & $0.51(0.45-0.60)$ \\
\hline
\end{tabular}


Table S3. Mean (95\% confidence interval) $R^{2}$ for predicting the most recent lab result using the baseline, methylation data, and genotype data. Confidence intervals determined using bootstrapping. Activated Partial Thromboplastin Time (APTT); Point of care (POC); Pulmonary function test (PFT); Forced expiratory volume in 1 second (FEV1)

\begin{tabular}{|c|c|c|c|}
\hline Lab Test & Baseline & Methylation & Genotypes \\
\hline Troponin & $0.65(0.53-0.74)$ & $0.62(0.51-0.72)$ & $0.60(0.50-0.70)$ \\
\hline Creatinine & $0.08(0.01-0.13)$ & $0.43(0.38-0.47)$ & $0.09(0.04-0.13)$ \\
\hline Troponin interpretation & $0.46(0.31-0.62)$ & $0.41(0.26-0.54)$ & $0.33(0.20-0.48)$ \\
\hline Urea nitrogen & $0.01(-0.04-0.05)$ & $0.40(0.35-0.45)$ & $0.03(-0.00-0.05)$ \\
\hline Absolute eosinophil count & $-0.06(-0.10-0.03)$ & $0.33(0.27-0.40)$ & $-0.01(-0.02-0.00)$ \\
\hline Hemoglobin & $0.10(0.04-0.15)$ & $0.28(0.23-0.33)$ & $0.10(0.05-0.14)$ \\
\hline Neutrophil percent (auto) & $0.23(0.12-0.32)$ & $0.26(0.18-0.33)$ & $0.22(0.13-0.30)$ \\
\hline PFT FEV1 (pre) & $0.12(-0.18-0.36)$ & $0.26(0.07-0.38)$ & $0.18(-0.02-0.32)$ \\
\hline Hematocrit & $0.07(0.02-0.11)$ & $0.24(0.20-0.28)$ & $0.07(0.04-0.11)$ \\
\hline Mean corpuscular hemoglobin & $0.07(0.01-0.13)$ & $0.20(0.15-0.26)$ & $0.08(0.04-0.12)$ \\
\hline Mean corpuscular volume & $0.09(0.03-0.14)$ & $0.18(0.12-0.24)$ & $0.09(0.04-0.13)$ \\
\hline Absolute lymphocyte count & $0.06(-0.04-0.17)$ & $0.17(0.08-0.33)$ & $0.10(0.03-0.23)$ \\
\hline Platelet count (auto) & $-0.00(-0.05-0.05)$ & $0.16(0.12-0.21)$ & $0.02(-0.00-0.04)$ \\
\hline Absolute neutrophil count & $0.08(0.01-0.13)$ & $0.15(0.10-0.20)$ & $0.08(0.04-0.11)$ \\
\hline Albumin & $0.07(0.01-0.13)$ & $0.14(0.08-0.18)$ & $0.08(0.04-0.12)$ \\
\hline Chloride & $-0.01(-0.05-0.03)$ & $0.13(0.09-0.18)$ & $0.01(-0.01-0.03)$ \\
\hline Absolute immature granulocyte count & $-0.09(-0.25-0.01)$ & $0.13(0.05-0.20)$ & $-0.00(-0.04-0.02)$ \\
\hline Absolute monocyte count & $0.08(0.00-0.14)$ & $0.12(0.06-0.17)$ & $0.07(0.01-0.13)$ \\
\hline White blood cell count & $-0.01(-0.07-0.04)$ & $0.11(0.05-0.19)$ & $0.02(-0.01-0.05)$ \\
\hline Neutrophils absolute (prelim). & $0.07(0.01-0.13)$ & $0.11(0.06-0.18)$ & $0.08(0.03-0.13)$ \\
\hline HgbA1C & $-0.07(-0.15-0.01)$ & $0.11(0.06-0.17)$ & $-0.01(-0.04-0.01)$ \\
\hline Total protein & $0.09(0.03-0.14)$ & $0.11(0.06-0.16)$ & $0.08(0.04-0.12)$ \\
\hline Sodium & $-0.00(-0.04-0.04)$ & $0.10(0.07-0.14)$ & $0.03(0.00-0.05)$ \\
\hline Ferritin & $-0.21(-0.39-0.07)$ & $0.10(0.04-0.15)$ & $-0.02(-0.05-0.00)$ \\
\hline Sedimentation rate erythrocyte & $-0.10(-0.38-0.12)$ & $0.10(-0.01-0.18)$ & $0.08(-0.01-0.17)$ \\
\hline Iron binding capacity & $-0.10(-0.19-0.00)$ & $0.09(0.03-0.14)$ & $-0.00(-0.03-0.03)$ \\
\hline Absolute basophil count & $-0.10(-0.25-0.02)$ & $0.07(0.05-0.11)$ & $-0.01(-0.02-0.00)$ \\
\hline Glucose & $-0.02(-0.07-0.02)$ & $0.07(0.04-0.10)$ & $0.01(-0.01-0.02)$ \\
\hline Qrs.duration & $-0.04(-0.11-0.02)$ & $0.05(0.00-0.08)$ & $0.01(-0.01-0.02)$ \\
\hline Cholesterol HDL & $0.03(-0.07-0.12)$ & $0.04(-0.03-0.10)$ & $0.06(0.00-0.12)$ \\
\hline Hematocrit OSL & $-0.31(-0.81-0.09)$ & $0.04(-0.13-0.19)$ & $-0.05(-0.22-0.08)$ \\
\hline Cholesterol & $-0.05(-0.11-0.02)$ & $0.03(-0.02-0.08)$ & $0.01(-0.02-0.04)$ \\
\hline Ventricular rate & $-0.06(-0.14-0.01)$ & $0.03(-0.00-0.06)$ & $0.02(-0.01-0.04)$ \\
\hline Anion gap & $-0.04(-0.07-0.01)$ & $0.02(-0.00-0.05)$ & $-0.00(-0.02-0.01)$ \\
\hline Alanine aminotransferase & $-0.05(-0.09-0.02)$ & $0.02(0.01-0.04)$ & $-0.01(-0.01-0.00)$ \\
\hline $\mathrm{R}$ axis & $-0.03(-0.10-0.03)$ & $0.01(-0.01-0.04)$ & $0.00(-0.02-0.03)$ \\
\hline Magnesium & $-0.11(-0.19-0.04)$ & $0.01(-0.02-0.05)$ & $-0.00(-0.03-0.02)$ \\
\hline Alkaline phosphatase & $-0.02(-0.07-0.02)$ & $0.01(-0.01-0.03)$ & $0.00(-0.01-0.01)$ \\
\hline T.axis & $-0.08(-0.19-0.01)$ & $0.01(-0.02-0.04)$ & $-0.00(-0.01-0.01)$ \\
\hline Cholesterol LDL (calculated) & $-0.10(-0.22-0.02)$ & $0.01(-0.02-0.04)$ & $-0.01(-0.03-0.01)$ \\
\hline Potassium & $-0.03(-0.07-0.00)$ & $0.01(-0.01-0.02)$ & $-0.01(-0.02-0.01)$ \\
\hline Aspartate aminotransferase & $-0.02(-0.07-0.03)$ & $0.01(-0.01-0.03)$ & $0.00(-0.02-0.02)$ \\
\hline
\end{tabular}


Table S3. Mean (95\% confidence interval) $R^{2}$ for predicting the most recent lab result using the baseline, methylation data, and genotype data. Confidence intervals determined using bootstrapping. Activated Partial Thromboplastin Time (APTT); Point of care (POC); Pulmonary function test (PFT); Forced expiratory volume in 1 second (FEV1)

\begin{tabular}{llll}
\hline Lab Test & Baseline & Methylation & Genotypes \\
\hline International normalized ratio (INR) & $-0.02(-0.06-0.02)$ & $0.01(-0.01-0.03)$ & $0.00(-0.02-0.02)$ \\
Bilirubin total & $-0.05(-0.16-0.01)$ & $0.01(-0.02-0.02)$ & $-0.00(-0.02-0.00)$ \\
Prothrombin time & $-0.02(-0.10-0.02)$ & $0.01(-0.01-0.03)$ & $0.00(-0.01-0.02)$ \\
QT interval & $-0.07(-0.12-0.01)$ & $0.00(-0.02-0.02)$ & $0.01(-0.01-0.02)$ \\
Glucose (POC) & $-0.08(-0.21-0.02)$ & $0.00(-0.03-0.03)$ & $0.00(-0.04-0.04)$ \\
X saturation & $-0.18(-0.30-0.06)$ & $0.00(-0.04-0.04)$ & $-0.01(-0.03-0.00)$ \\
PR interval & $-0.05(-0.15-0.03)$ & $0.00(-0.04-0.03)$ & $-0.00(-0.03-0.02)$ \\
Brain natriuretic peptide (BNP) & $-0.51(-1.38-0.03)$ & $0.00(-0.08-0.05)$ & $0.03(-0.07-0.11)$ \\
Glucose whole blood & $-0.24(-0.49-0.09)$ & $-0.00(-0.03-0.03)$ & $-0.03(-0.08-0.00)$ \\
Atrial rate & $-0.05(-0.13-0.01)$ & $-0.00(-0.02-0.01)$ & $-0.00(-0.02-0.02)$ \\
P axis & $-0.04(-0.10-0.01)$ & $-0.00(-0.02-0.02)$ & $-0.00(-0.02-0.02)$ \\
QtC calculation (bezet) & $-0.07(-0.14-0.01)$ & $-0.00(-0.02-0.02)$ & $-0.00(-0.02-0.01)$ \\
PFT FEV1 (pre) (percent ref) & $-0.31(-0.58-0.06)$ & $-0.00(-0.06-0.04)$ & $-0.03(-0.07-0.01)$ \\
Blood lactate & $-0.29(-0.64-0.02)$ & $-0.01(-0.08-0.05)$ & $0.00(-0.06-0.05)$ \\
APTT & $-0.05(-0.12-0.01)$ & $-0.01(-0.02-0.00)$ & $-0.01(-0.03-0.00)$ \\
Triglycerides & $-0.13(-0.23-0.05)$ & $-0.01(-0.03-0.01)$ & $-0.01(-0.03-0.00)$ \\
Thyroid stimulating hormone (TSH) & $-0.13(-0.37-0.05)$ & $-0.01(-0.04-0.01)$ & $-0.02(-0.04-0.00)$ \\
Calcium & $-0.05(-0.08-0.02)$ & $-0.01(-0.02-0.00)$ & $-0.02(-0.03-0.00)$ \\
Iron & $-0.13(-0.26-0.00)$ & $-0.02(-0.06-0.03)$ & $-0.02(-0.05-0.01)$ \\
Urea nitrogen (OSL) & $-0.61(-1.93-0.00)$ & $-0.04(-0.17-0.05)$ & $-0.04(-0.22-0.03)$ \\
Bilirubin conjugated & $-0.57(-1.25-0.17)$ & $-0.06(-0.18-0.00)$ & $-0.06(-0.17-0.03)$ \\
C reactive protein (CRP) & $-0.44(-1.14-0.13)$ & $-0.06(-0.24-0.01)$ & $-0.01(-0.08-0.02)$ \\
Left ventricular ejection fraction & $-1.80(-3.29-0.91)$ & $-0.06(-0.21-0.01)$ & $-0.07(-0.28-0.03)$ \\
Hemoglobin (OSL) & $-2.66(-12.80-0.23)$ & $-0.07(-0.37-0.02)$ & $-0.09(-0.45-0.02)$ \\
Chloride (OSL) & $-1.96(-4.43-0.46)$ & $-0.19(-0.70-0.03)$ & $-0.17(-0.65-0.04)$ \\
Sodium (OSL) & $-304.86(-2851.16-0.13)$ & $-2.03(-16.23-0.02)$ & $-1.57(-16.42-0.01)$ \\
Potassium (OSL). & $-178.77(-828.09-0.14)$ & $-2.33(-8.10-0.02)$ & $-2.42(-8.14-0.02)$ \\
\hline
\end{tabular}

Table S4. Mean (95\% confidence interval) area under the ROC curve for predicting patient diagnoses, grouped into Phecode phenotypes, using the baseline, methylation data, and genotype data. Confidence intervals determined using bootstrapping.

\begin{tabular}{llll}
\hline Phecode Phenotype & Baseline & Methylation & Genotypes \\
\hline End stage renal disease & $0.72(0.68-0.76)$ & $0.90(0.87-0.92)$ & $0.72(0.67-0.77)$ \\
Renal dialysis & $0.73(0.67-0.77)$ & $0.88(0.85-0.91)$ & $0.72(0.68-0.76)$ \\
Cirrhosis of liver without mention of alcohol & $0.69(0.63-0.76)$ & $0.86(0.82-0.89)$ & $0.68(0.62-0.75)$ \\
Morbid obesity & $0.86(0.79-0.92)$ & $0.85(0.78-0.90)$ & $0.86(0.79-0.91)$ \\
Cancer of prostate & $0.82(0.76-0.86)$ & $0.85(0.78-0.89)$ & $0.78(0.73-0.83)$ \\
Hyperplasia of prostate & $0.85(0.81-0.88)$ & $0.84(0.82-0.87)$ & $0.84(0.81-0.88)$ \\
Neutropenia & $0.60(0.51-0.67)$ & $0.84(0.79-0.88)$ & $0.42(0.36-0.49)$ \\
Obesity & $0.84(0.80-0.87)$ & $0.84(0.80-0.87)$ & $0.84(0.80-0.87)$ \\
\hline
\end{tabular}


Table S4. Mean (95\% confidence interval) area under the ROC curve for predicting patient diagnoses, grouped into Phecode phenotypes, using the baseline, methylation data, and genotype data. Confidence intervals determined using bootstrapping.

\begin{tabular}{|c|c|c|c|}
\hline Phecode Phenotype & Baseline & Methylation & Genotypes \\
\hline Menopausal and postmenopausal disorders & $0.84(0.80-0.87)$ & $0.83(0.79-0.87)$ & $0.82(0.78-0.86)$ \\
\hline Tobacco use disorder & $0.79(0.72-0.84)$ & $0.83(0.77-0.88)$ & $0.77(0.72-0.82)$ \\
\hline Noninflammatory female genital disorders & $0.80(0.77-0.83)$ & $0.82(0.78-0.85)$ & $0.78(0.74-0.81)$ \\
\hline Chronic renal failure $[\mathrm{CKD}]$ & $0.63(0.59-0.68)$ & $0.82(0.78-0.85)$ & $0.63(0.58-0.66)$ \\
\hline Immunity deficiency & $0.76(0.70-0.80)$ & $0.82(0.77-0.86)$ & $0.75(0.70-0.80)$ \\
\hline Poisoning by primarily systemic agents & $0.63(0.55-0.71)$ & $0.82(0.74-0.88)$ & $0.55(0.46-0.62)$ \\
\hline Kidney replaced by transpant & $0.74(0.69-0.79)$ & $0.81(0.77-0.86)$ & $0.72(0.67-0.78)$ \\
\hline Decreased white blood cell count & $0.66(0.59-0.71)$ & $0.81(0.76-0.86)$ & $0.62(0.56-0.68)$ \\
\hline Anemia in chronic kidney disease & $0.71(0.65-0.76)$ & $0.81(0.77-0.85)$ & $0.71(0.65-0.77)$ \\
\hline Portal hypertension & $0.70(0.63-0.76)$ & $0.80(0.73-0.86)$ & $0.68(0.60-0.75)$ \\
\hline Disorders involving the immune mechanism & $0.76(0.72-0.80)$ & $0.80(0.76-0.84)$ & $0.75(0.70-0.79)$ \\
\hline Hypertensive chronic kidney disease & $0.62(0.57-0.66)$ & $0.80(0.76-0.84)$ & $0.59(0.55-0.64)$ \\
\hline Renal failure & $0.62(0.58-0.66)$ & $0.80(0.76-0.83)$ & $0.60(0.57-0.64)$ \\
\hline Disorders resulting from impaired renal function & $0.68(0.62-0.74)$ & $0.79(0.74-0.84)$ & $0.68(0.61-0.73)$ \\
\hline Antineoplastic and immunosuppressive drugs caus... & $0.67(0.59-0.75)$ & $0.79(0.71-0.87)$ & $0.48(0.41-0.58)$ \\
\hline Anemia of chronic disease & $0.70(0.65-0.73)$ & $0.79(0.76-0.82)$ & $0.70(0.65-0.74)$ \\
\hline Overweight, obesity and other hyperalimentation & $0.79(0.75-0.83)$ & $0.79(0.75-0.83)$ & $0.79(0.75-0.83)$ \\
\hline Liver abscess and sequelae of chronic liver dis... & $0.72(0.64-0.79)$ & $0.78(0.72-0.84)$ & $0.66(0.58-0.73)$ \\
\hline Osteoarthrosis & $0.78(0.73-0.82)$ & $0.78(0.73-0.82)$ & $0.77(0.73-0.81)$ \\
\hline Liver replaced by transplant & $0.68(0.61-0.77)$ & $0.76(0.70-0.82)$ & $0.64(0.56-0.74)$ \\
\hline Secondary hyperparathyroidism (of renal origin) & $0.67(0.60-0.73)$ & $0.76(0.71-0.82)$ & $0.65(0.57-0.71)$ \\
\hline Osteoarthrosis NOS & $0.77(0.72-0.82)$ & $0.76(0.71-0.81)$ & $0.76(0.72-0.81)$ \\
\hline Disorders of phosphorus metabolism & $0.73(0.65-0.80)$ & $0.76(0.69-0.82)$ & $0.71(0.63-0.78)$ \\
\hline Abnormal involuntary movements & $0.39(0.31-0.49)$ & $0.76(0.68-0.82)$ & $0.54(0.44-0.62)$ \\
\hline Fluid overload & $0.66(0.61-0.73)$ & $0.76(0.70-0.81)$ & $0.68(0.62-0.75)$ \\
\hline Erectile dysfunction $[\mathrm{ED}]$ & $0.72(0.65-0.78)$ & $0.76(0.70-0.82)$ & $0.72(0.64-0.77)$ \\
\hline Osteoarthritis; localized & $0.76(0.71-0.82)$ & $0.76(0.70-0.82)$ & $0.74(0.68-0.80)$ \\
\hline Coagulation defects & $0.68(0.62-0.75)$ & $0.74(0.67-0.80)$ & $0.64(0.56-0.72)$ \\
\hline Acid-base balance disorder & $0.66(0.60-0.72)$ & $0.73(0.68-0.79)$ & $0.64(0.58-0.71)$ \\
\hline Osteoarthrosis, localized, primary & $0.75(0.69-0.81)$ & $0.73(0.66-0.78)$ & $0.75(0.69-0.80)$ \\
\hline Respiratory failure, insufficiency, arrest & $0.61(0.55-0.67)$ & $0.73(0.68-0.78)$ & $0.56(0.48-0.64)$ \\
\hline Ascites (non malignant) & $0.73(0.66-0.80)$ & $0.73(0.65-0.79)$ & $0.73(0.66-0.79)$ \\
\hline Degenerative skin conditions and other dermatoses & $0.72(0.67-0.76)$ & $0.73(0.68-0.78)$ & $0.70(0.65-0.75)$ \\
\hline Hypertensive heart and/or renal disease & $0.56(0.51-0.61)$ & $0.72(0.69-0.77)$ & $0.58(0.53-0.63)$ \\
\hline Altered mental status & $0.62(0.53-0.71)$ & $0.72(0.64-0.81)$ & $0.59(0.50-0.68)$ \\
\hline Arthropathy NOS & $0.72(0.66-0.78)$ & $0.72(0.66-0.78)$ & $0.71(0.65-0.77)$ \\
\hline Cataract & $0.73(0.68-0.77)$ & $0.72(0.67-0.76)$ & $0.72(0.67-0.76)$ \\
\hline Nephritis and nephropathy in diseases classifie... & $0.69(0.61-0.76)$ & $0.71(0.64-0.78)$ & $0.70(0.63-0.77)$ \\
\hline Family history & $0.64(0.56-0.71)$ & $0.71(0.65-0.79)$ & $0.66(0.56-0.75)$ \\
\hline Purpura and other hemorrhagic conditions & $0.61(0.53-0.68)$ & $0.71(0.65-0.78)$ & $0.61(0.54-0.67)$ \\
\hline Chronic liver disease and cirrhosis & $0.61(0.55-0.68)$ & $0.71(0.65-0.77)$ & $0.62(0.55-0.68)$ \\
\hline Osteoporosis NOS & $0.72(0.64-0.79)$ & $0.71(0.64-0.78)$ & $0.71(0.64-0.78)$ \\
\hline Splenomegaly & $0.65(0.59-0.71)$ & $0.71(0.63-0.77)$ & $0.65(0.57-0.73)$ \\
\hline
\end{tabular}


Table S4. Mean (95\% confidence interval) area under the ROC curve for predicting patient diagnoses, grouped into Phecode phenotypes, using the baseline, methylation data, and genotype data. Confidence intervals determined using bootstrapping.

\begin{tabular}{|c|c|c|c|}
\hline Phecode Phenotype & Baseline & Methylation & Genotypes \\
\hline Hyperpotassemia & $0.66(0.61-0.71)$ & $0.71(0.66-0.75)$ & $0.64(0.59-0.69)$ \\
\hline Disorders of calcium/phosphorus metabolism & $0.67(0.58-0.73)$ & $0.71(0.65-0.76)$ & $0.65(0.59-0.72)$ \\
\hline Other disorders of metabolism & $0.57(0.48-0.65)$ & $0.71(0.63-0.77)$ & $0.52(0.43-0.59)$ \\
\hline Viral infection & $0.68(0.61-0.75)$ & $0.70(0.64-0.77)$ & $0.66(0.58-0.73)$ \\
\hline Acute renal failure & $0.60(0.55-0.64)$ & $0.70(0.66-0.74)$ & $0.57(0.53-0.62)$ \\
\hline Essential hypertension & $0.67(0.64-0.71)$ & $0.70(0.66-0.74)$ & $0.67(0.64-0.70)$ \\
\hline Seborrheic keratosis & $0.70(0.65-0.75)$ & $0.70(0.65-0.75)$ & $0.68(0.63-0.73)$ \\
\hline Diverticulosis and diverticulitis & $0.72(0.65-0.78)$ & $0.70(0.64-0.75)$ & $0.68(0.60-0.75)$ \\
\hline Hyperlipidemia & $0.70(0.66-0.74)$ & $0.70(0.66-0.73)$ & $0.70(0.66-0.74)$ \\
\hline Thrombocytopenia & $0.62(0.56-0.69)$ & $0.70(0.64-0.76)$ & $0.63(0.56-0.69)$ \\
\hline Type 2 diabetes & $0.66(0.62-0.71)$ & $0.69(0.65-0.73)$ & $0.65(0.62-0.69)$ \\
\hline Other ill-defined and unknown causes of morbidi... & $0.72(0.65-0.78)$ & $0.69(0.63-0.77)$ & $0.68(0.60-0.75)$ \\
\hline Osteoporosis & $0.72(0.65-0.78)$ & $0.69(0.62-0.76)$ & $0.68(0.61-0.76)$ \\
\hline Poisoning by hormones and synthetic substitutes & $0.60(0.51-0.70)$ & $0.69(0.61-0.77)$ & $0.56(0.47-0.65)$ \\
\hline Renal failure NOS & $0.61(0.55-0.69)$ & $0.69(0.60-0.76)$ & $0.56(0.48-0.62)$ \\
\hline Type 2 diabetes with neurological manifestations & $0.67(0.60-0.74)$ & $0.69(0.62-0.76)$ & $0.65(0.56-0.73)$ \\
\hline Polyneuropathy in diabetes & $0.64(0.57-0.71)$ & $0.69(0.60-0.77)$ & $0.62(0.53-0.70)$ \\
\hline Respiratory failure & $0.65(0.58-0.72)$ & $0.69(0.61-0.76)$ & $0.61(0.53-0.68)$ \\
\hline Other immunological findings & $0.65(0.56-0.73)$ & $0.69(0.61-0.77)$ & $0.62(0.54-0.71)$ \\
\hline Deep vein thrombosis $[\mathrm{DVT}]$ & $0.67(0.59-0.74)$ & $0.69(0.63-0.76)$ & $0.61(0.53-0.69)$ \\
\hline Carditis & $0.60(0.51-0.69)$ & $0.69(0.61-0.76)$ & $0.41(0.34-0.50)$ \\
\hline Other disorders of the kidney and ureters & $0.56(0.51-0.60)$ & $0.69(0.65-0.72)$ & $0.54(0.49-0.60)$ \\
\hline Skin cancer & $0.71(0.66-0.75)$ & $0.69(0.63-0.74)$ & $0.69(0.64-0.75)$ \\
\hline Other non-epithelial cancer of skin & $0.71(0.65-0.76)$ & $0.68(0.63-0.74)$ & $0.68(0.64-0.74)$ \\
\hline Cardiac pacemaker/device in situ & $0.43(0.36-0.51)$ & $0.68(0.62-0.75)$ & $0.61(0.53-0.69)$ \\
\hline Type 2 diabetes with renal manifestations & $0.63(0.57-0.68)$ & $0.68(0.62-0.74)$ & $0.65(0.58-0.71)$ \\
\hline Ischemic Heart Disease & $0.68(0.64-0.72)$ & $0.68(0.64-0.72)$ & $0.67(0.64-0.71)$ \\
\hline Protein-calorie malnutrition & $0.67(0.59-0.73)$ & $0.68(0.62-0.74)$ & $0.65(0.59-0.71)$ \\
\hline Nephritis and nephropathy without mention of gl. & $0.59(0.52-0.66)$ & $0.68(0.63-0.74)$ & $0.61(0.54-0.67)$ \\
\hline Coronary atherosclerosis & $0.68(0.65-0.72)$ & $0.68(0.65-0.72)$ & $0.68(0.64-0.71)$ \\
\hline Disorders of diaphragm & $0.66(0.59-0.73)$ & $0.68(0.60-0.75)$ & $0.64(0.56-0.71)$ \\
\hline Myocardial infarction & $0.66(0.60-0.72)$ & $0.68(0.63-0.73)$ & $0.65(0.60-0.71)$ \\
\hline Other and unspecified coagulation defects & $0.59(0.52-0.67)$ & $0.68(0.60-0.75)$ & $0.50(0.43-0.58)$ \\
\hline Intestinal infection & $0.66(0.58-0.74)$ & $0.68(0.60-0.75)$ & $0.62(0.52-0.72)$ \\
\hline Chronic Kidney Disease, Stage IV & $0.55(0.48-0.62)$ & $0.68(0.61-0.75)$ & $0.49(0.43-0.55)$ \\
\hline Sensorineural hearing loss & $0.71(0.62-0.79)$ & $0.68(0.58-0.77)$ & $0.67(0.58-0.76)$ \\
\hline Other arthropathies & $0.70(0.64-0.76)$ & $0.68(0.61-0.74)$ & $0.69(0.63-0.76)$ \\
\hline Diverticulosis & $0.71(0.62-0.79)$ & $0.68(0.59-0.77)$ & $0.70(0.60-0.78)$ \\
\hline Pneumonia & $0.67(0.62-0.73)$ & $0.67(0.63-0.72)$ & $0.66(0.60-0.71)$ \\
\hline Glaucoma & $0.67(0.61-0.74)$ & $0.67(0.61-0.73)$ & $0.65(0.59-0.72)$ \\
\hline Postinflammatory pulmonary fibrosis & $0.66(0.57-0.75)$ & $0.67(0.59-0.75)$ & $0.59(0.51-0.68)$ \\
\hline Hypertension & $0.65(0.61-0.69)$ & $0.67(0.64-0.71)$ & $0.64(0.60-0.68)$ \\
\hline Sleep apnea & $0.66(0.61-0.73)$ & $0.67(0.61-0.72)$ & $0.67(0.61-0.72)$ \\
\hline
\end{tabular}


Table S4. Mean (95\% confidence interval) area under the ROC curve for predicting patient diagnoses, grouped into Phecode phenotypes, using the baseline, methylation data, and genotype data. Confidence intervals determined using bootstrapping.

\begin{tabular}{|c|c|c|c|}
\hline Phecode Phenotype & Baseline & Methylation & Genotypes \\
\hline Aortic aneurysm & $0.68(0.61-0.74)$ & $0.67(0.58-0.75)$ & $0.65(0.57-0.73)$ \\
\hline Septicemia & $0.66(0.60-0.72)$ & $0.67(0.63-0.73)$ & $0.63(0.57-0.69)$ \\
\hline Disorders of lipoid metabolism & $0.67(0.63-0.70)$ & $0.67(0.63-0.71)$ & $0.67(0.64-0.71)$ \\
\hline Iron deficiency anemia secondary to blood loss ... & $0.51(0.44-0.59)$ & $0.67(0.59-0.74)$ & $0.49(0.42-0.56)$ \\
\hline Actinic keratosis & $0.69(0.62-0.75)$ & $0.67(0.60-0.73)$ & $0.73(0.67-0.79)$ \\
\hline Other anemias & $0.62(0.58-0.66)$ & $0.67(0.63-0.71)$ & $0.62(0.58-0.66)$ \\
\hline Abnormal movement & $0.65(0.59-0.71)$ & $0.67(0.61-0.73)$ & $0.63(0.57-0.69)$ \\
\hline Disorders of fluid, electrolyte, and acid-base ... & $0.62(0.58-0.67)$ & $0.67(0.62-0.71)$ & $0.63(0.59-0.67)$ \\
\hline Acidosis & $0.64(0.58-0.68)$ & $0.67(0.61-0.72)$ & $0.61(0.54-0.67)$ \\
\hline Sepsis & $0.63(0.58-0.69)$ & $0.67(0.61-0.72)$ & $0.60(0.53-0.65)$ \\
\hline Atherosclerosis & $0.53(0.47-0.60)$ & $0.66(0.61-0.73)$ & $0.45(0.39-0.52)$ \\
\hline Nephritis; nephrosis; renal sclerosis & $0.56(0.49-0.63)$ & $0.66(0.61-0.72)$ & $0.58(0.51-0.63)$ \\
\hline Osteoporosis, osteopenia and pathological fracture & $0.64(0.59-0.68)$ & $0.66(0.62-0.71)$ & $0.63(0.59-0.68)$ \\
\hline Other disorders of intestine & $0.58(0.49-0.66)$ & $0.66(0.57-0.74)$ & $0.51(0.43-0.60)$ \\
\hline Tachycardia NOS & $0.62(0.55-0.68)$ & $0.66(0.60-0.72)$ & $0.59(0.52-0.66)$ \\
\hline Iron deficiency anemias, unspecified or not due... & $0.67(0.61-0.72)$ & $0.66(0.61-0.71)$ & $0.70(0.63-0.76)$ \\
\hline Other retinal disorders & $0.63(0.55-0.71)$ & $0.66(0.57-0.73)$ & $0.57(0.49-0.64)$ \\
\hline Effects radiation NOS & $0.64(0.55-0.71)$ & $0.66(0.58-0.74)$ & $0.59(0.52-0.69)$ \\
\hline Diabetes mellitus & $0.65(0.61-0.69)$ & $0.66(0.62-0.70)$ & $0.64(0.61-0.68)$ \\
\hline Other disorders of eye & $0.69(0.64-0.75)$ & $0.66(0.59-0.72)$ & $0.69(0.62-0.76)$ \\
\hline Disorders of vitreous body & $0.67(0.60-0.75)$ & $0.66(0.59-0.73)$ & $0.69(0.62-0.76)$ \\
\hline Emphysema & $0.66(0.58-0.73)$ & $0.66(0.58-0.72)$ & $0.62(0.54-0.71)$ \\
\hline Senile cataract & $0.69(0.64-0.73)$ & $0.66(0.60-0.72)$ & $0.67(0.61-0.71)$ \\
\hline Nonrheumatic aortic valve disorders & $0.66(0.61-0.71)$ & $0.66(0.60-0.71)$ & $0.65(0.60-0.70)$ \\
\hline Hypotension & $0.63(0.58-0.69)$ & $0.66(0.60-0.71)$ & $0.61(0.55-0.66)$ \\
\hline Electrolyte imbalance & $0.64(0.60-0.68)$ & $0.66(0.61-0.70)$ & $0.62(0.58-0.67)$ \\
\hline Atherosclerosis of aorta & $0.67(0.62-0.72)$ & $0.66(0.60-0.71)$ & $0.65(0.60-0.70)$ \\
\hline Sepsis and SIRS & $0.65(0.59-0.71)$ & $0.65(0.60-0.71)$ & $0.63(0.57-0.69)$ \\
\hline Acute posthemorrhagic anemia & $0.67(0.62-0.72)$ & $0.65(0.59-0.70)$ & $0.66(0.61-0.70)$ \\
\hline Urinary tract infection & $0.62(0.57-0.67)$ & $0.65(0.61-0.70)$ & $0.62(0.57-0.66)$ \\
\hline Other chronic ischemic heart disease, unspecified & $0.70(0.62-0.77)$ & $0.65(0.58-0.72)$ & $0.67(0.60-0.74)$ \\
\hline Nausea and vomiting & $0.53(0.48-0.59)$ & $0.65(0.61-0.69)$ & $0.47(0.41-0.52)$ \\
\hline Chronic Kidney Disease, Stage III & $0.58(0.52-0.63)$ & $0.65(0.60-0.70)$ & $0.55(0.49-0.62)$ \\
\hline Fever of unknown origin & $0.63(0.58-0.68)$ & $0.65(0.59-0.71)$ & $0.63(0.55-0.69)$ \\
\hline Acute pain & $0.59(0.53-0.65)$ & $0.65(0.60-0.71)$ & $0.58(0.52-0.64)$ \\
\hline Obstructive sleep apnea & $0.65(0.59-0.71)$ & $0.65(0.58-0.72)$ & $0.65(0.58-0.71)$ \\
\hline Alcoholism & $0.60(0.49-0.70)$ & $0.65(0.57-0.74)$ & $0.52(0.41-0.62)$ \\
\hline Secondary malignant neoplasm & $0.56(0.48-0.65)$ & $0.65(0.57-0.72)$ & $0.66(0.58-0.73)$ \\
\hline Cystitis and urethritis & $0.59(0.53-0.66)$ & $0.65(0.57-0.72)$ & $0.55(0.47-0.63)$ \\
\hline Angina pectoris & $0.64(0.59-0.70)$ & $0.65(0.58-0.70)$ & $0.59(0.53-0.65)$ \\
\hline Urinary incontinence & $0.65(0.59-0.73)$ & $0.65(0.57-0.73)$ & $0.63(0.56-0.69)$ \\
\hline Hypercholesterolemia & $0.64(0.59-0.68)$ & $0.65(0.60-0.69)$ & $0.63(0.57-0.67)$ \\
\hline Articular cartilage disorder & $0.64(0.57-0.69)$ & $0.65(0.58-0.71)$ & $0.61(0.54-0.68)$ \\
\hline
\end{tabular}


Table S4. Mean (95\% confidence interval) area under the ROC curve for predicting patient diagnoses, grouped into Phecode phenotypes, using the baseline, methylation data, and genotype data. Confidence intervals determined using bootstrapping.

\begin{tabular}{|c|c|c|c|}
\hline Phecode Phenotype & Baseline & Methylation & Genotypes \\
\hline Aortic valve disease & $0.65(0.59-0.70)$ & $0.64(0.59-0.70)$ & $0.56(0.50-0.61)$ \\
\hline Enthesopathy & $0.66(0.58-0.72)$ & $0.64(0.57-0.71)$ & $0.63(0.55-0.71)$ \\
\hline Heart failure with preserved EF [Diastolic hear... & $0.66(0.58-0.74)$ & $0.64(0.56-0.73)$ & $0.55(0.47-0.63)$ \\
\hline Hemorrhage of gastrointestinal tract & $0.66(0.57-0.75)$ & $0.64(0.57-0.72)$ & $0.50(0.40-0.58)$ \\
\hline Bacterial infection NOS & $0.62(0.56-0.68)$ & $0.64(0.59-0.70)$ & $0.60(0.54-0.65)$ \\
\hline Osteopenia or other disorder of bone and cartilage & $0.64(0.59-0.69)$ & $0.64(0.59-0.69)$ & $0.63(0.58-0.68)$ \\
\hline Disorders of protein plasma/amino-acid transpor... & $0.65(0.57-0.74)$ & $0.64(0.57-0.72)$ & $0.62(0.55-0.69)$ \\
\hline Cardiac shunt/ heart septal defect & $0.62(0.54-0.71)$ & $0.64(0.55-0.72)$ & $0.54(0.44-0.63)$ \\
\hline Hyposmolality and/or hyponatremia & $0.61(0.56-0.66)$ & $0.64(0.59-0.69)$ & $0.60(0.56-0.65)$ \\
\hline Heart valve disorders & $0.64(0.59-0.68)$ & $0.64(0.60-0.68)$ & $0.62(0.57-0.67)$ \\
\hline Disorders of mineral metabolism & $0.63(0.58-0.68)$ & $0.64(0.59-0.69)$ & $0.62(0.56-0.68)$ \\
\hline Pain in joint & $0.63(0.58-0.68)$ & $0.64(0.59-0.68)$ & $0.61(0.57-0.65)$ \\
\hline Pulmonary collapse; interstitial and compensato... & $0.60(0.54-0.66)$ & $0.64(0.57-0.71)$ & $0.55(0.49-0.62)$ \\
\hline Other forms of chronic heart disease & $0.49(0.40-0.57)$ & $0.64(0.56-0.71)$ & $0.49(0.41-0.58)$ \\
\hline Other venous embolism and thrombosis & $0.57(0.51-0.64)$ & $0.64(0.58-0.71)$ & $0.55(0.48-0.61)$ \\
\hline Complications of transplants and reattached limbs & $0.63(0.55-0.72)$ & $0.63(0.54-0.72)$ & $0.61(0.51-0.69)$ \\
\hline Edema & $0.59(0.53-0.64)$ & $0.63(0.58-0.68)$ & $0.58(0.53-0.63)$ \\
\hline Benign neoplasm of colon & $0.59(0.53-0.64)$ & $0.63(0.58-0.69)$ & $0.58(0.53-0.65)$ \\
\hline Symptoms concerning nutrition, metabolism, and & $0.61(0.56-0.67)$ & $0.63(0.57-0.69)$ & $0.59(0.53-0.66)$ \\
\hline Other disorders of bladder & $0.62(0.55-0.70)$ & $0.63(0.55-0.70)$ & $0.59(0.51-0.66)$ \\
\hline Chronic pulmonary heart disease & $0.57(0.50-0.63)$ & $0.63(0.57-0.70)$ & $0.55(0.47-0.61)$ \\
\hline Diseases of the larynx and vocal cords & $0.49(0.40-0.58)$ & $0.63(0.56-0.70)$ & $0.40(0.32-0.48)$ \\
\hline Chronic airway obstruction & $0.64(0.58-0.70)$ & $0.63(0.56-0.69)$ & $0.61(0.55-0.69)$ \\
\hline Candidiasis & $0.63(0.56-0.70)$ & $0.63(0.56-0.69)$ & $0.61(0.54-0.68)$ \\
\hline Neoplasm of uncertain behavior & $0.58(0.49-0.66)$ & $0.63(0.55-0.70)$ & $0.41(0.33-0.50)$ \\
\hline Shortness of breath & $0.62(0.57-0.67)$ & $0.63(0.58-0.67)$ & $0.61(0.56-0.65)$ \\
\hline Degeneration of intervertebral disc & $0.63(0.57-0.70)$ & $0.62(0.56-0.69)$ & $0.61(0.54-0.67)$ \\
\hline Symptoms involving respiratory system and other... & $0.54(0.45-0.64)$ & $0.62(0.54-0.70)$ & $0.62(0.52-0.70)$ \\
\hline Peripheral vascular disease & $0.65(0.59-0.70)$ & $0.62(0.57-0.67)$ & $0.61(0.55-0.66)$ \\
\hline Heart failure NOS & $0.55(0.50-0.61)$ & $0.62(0.56-0.68)$ & $0.49(0.44-0.55)$ \\
\hline Cardiomegaly & $0.52(0.48-0.57)$ & $0.62(0.58-0.67)$ & $0.49(0.45-0.54)$ \\
\hline Congenital anomalies of great vessels & $0.64(0.59-0.70)$ & $0.62(0.57-0.68)$ & $0.63(0.58-0.70)$ \\
\hline Pleurisy; pleural effusion & $0.60(0.56-0.65)$ & $0.62(0.58-0.67)$ & $0.58(0.54-0.63)$ \\
\hline Mixed hyperlipidemia & $0.63(0.57-0.68)$ & $0.62(0.56-0.68)$ & $0.61(0.55-0.66)$ \\
\hline Other aneurysm & $0.63(0.55-0.71)$ & $0.62(0.54-0.69)$ & $0.60(0.53-0.67)$ \\
\hline Abnormality of gait & $0.69(0.61-0.75)$ & $0.62(0.56-0.69)$ & $0.66(0.59-0.73)$ \\
\hline Atrial fibrillation and flutter & $0.64(0.59-0.69)$ & $0.62(0.57-0.66)$ & $0.63(0.59-0.68)$ \\
\hline Infection/inflammation of internal prosthetic d... & $0.59(0.51-0.67)$ & $0.62(0.54-0.71)$ & $0.60(0.51-0.68)$ \\
\hline Occlusion and stenosis of precerebral arteries & $0.69(0.61-0.76)$ & $0.62(0.54-0.70)$ & $0.58(0.51-0.66)$ \\
\hline Other chronic nonalcoholic liver disease & $0.60(0.55-0.67)$ & $0.62(0.56-0.68)$ & $0.61(0.54-0.68)$ \\
\hline Bacteremia & $0.61(0.54-0.70)$ & $0.61(0.53-0.70)$ & $0.58(0.48-0.67)$ \\
\hline Bacterial pneumonia & $0.64(0.55-0.72)$ & $0.61(0.52-0.70)$ & $0.66(0.58-0.74)$ \\
\hline Atrial fibrillation & $0.63(0.58-0.68)$ & $0.61(0.56-0.66)$ & $0.59(0.53-0.65)$ \\
\hline
\end{tabular}


Table S4. Mean (95\% confidence interval) area under the ROC curve for predicting patient diagnoses, grouped into Phecode phenotypes, using the baseline, methylation data, and genotype data. Confidence intervals determined using bootstrapping.

\begin{tabular}{|c|c|c|c|}
\hline Phecode Phenotype & Baseline & Methylation & Genotypes \\
\hline Substance addiction and disorders & $0.62(0.52-0.70)$ & $0.61(0.55-0.68)$ & $0.57(0.49-0.67)$ \\
\hline Contusion & $0.50(0.41-0.59)$ & $0.61(0.53-0.69)$ & $0.49(0.40-0.58)$ \\
\hline Inflammation of the eye & $0.65(0.58-0.71)$ & $0.61(0.51-0.69)$ & $0.59(0.52-0.67)$ \\
\hline Congestive heart failure (CHF) NOS & $0.59(0.54-0.65)$ & $0.61(0.55-0.67)$ & $0.53(0.47-0.58)$ \\
\hline Other tests & $0.54(0.46-0.62)$ & $0.61(0.53-0.68)$ & $0.59(0.50-0.65)$ \\
\hline Other diseases of blood and blood-forming organs & $0.58(0.50-0.66)$ & $0.61(0.53-0.70)$ & $0.47(0.38-0.56)$ \\
\hline Blood in stool & $0.54(0.46-0.64)$ & $0.61(0.51-0.68)$ & $0.39(0.32-0.47)$ \\
\hline Iron deficiency anemias & $0.63(0.56-0.70)$ & $0.61(0.54-0.67)$ & $0.60(0.53-0.67)$ \\
\hline Other hypertensive complications & $0.62(0.55-0.67)$ & $0.60(0.55-0.66)$ & $0.62(0.55-0.69)$ \\
\hline Hypertensive heart disease & $0.61(0.54-0.67)$ & $0.60(0.53-0.67)$ & $0.68(0.61-0.74)$ \\
\hline Hypovolemia & $0.56(0.48-0.66)$ & $0.60(0.51-0.69)$ & $0.47(0.37-0.55)$ \\
\hline Insulin pump user & $0.59(0.53-0.65)$ & $0.60(0.53-0.66)$ & $0.58(0.51-0.65)$ \\
\hline Primary/intrinsic cardiomyopathies & $0.55(0.50-0.61)$ & $0.60(0.53-0.68)$ & $0.46(0.40-0.51)$ \\
\hline Peripheral enthesopathies and allied syndromes & $0.61(0.55-0.68)$ & $0.60(0.54-0.66)$ & $0.57(0.51-0.63)$ \\
\hline Other diseases of lung & $0.55(0.50-0.60)$ & $0.60(0.54-0.65)$ & $0.52(0.47-0.57)$ \\
\hline Dermatophytosis / Dermatomycosis & $0.60(0.54-0.67)$ & $0.60(0.53-0.68)$ & $0.60(0.53-0.65)$ \\
\hline Shock & $0.59(0.50-0.66)$ & $0.60(0.52-0.69)$ & $0.51(0.42-0.59)$ \\
\hline Heart valve replaced & $0.56(0.49-0.63)$ & $0.60(0.53-0.67)$ & $0.51(0.43-0.57)$ \\
\hline Pain & $0.56(0.52-0.61)$ & $0.60(0.56-0.64)$ & $0.55(0.50-0.59)$ \\
\hline Dizziness and giddiness (Light-headedness and $\mathrm{v} . .$. & $0.61(0.54-0.67)$ & $0.60(0.53-0.65)$ & $0.59(0.53-0.64)$ \\
\hline Nonrheumatic mitral valve disorders & $0.58(0.53-0.64)$ & $0.60(0.54-0.65)$ & $0.56(0.51-0.62)$ \\
\hline Diseases of white blood cells & $0.52(0.42-0.59)$ & $0.60(0.53-0.66)$ & $0.41(0.35-0.48)$ \\
\hline Peripheral vascular disease, unspecified & $0.60(0.54-0.65)$ & $0.59(0.53-0.65)$ & $0.59(0.53-0.65)$ \\
\hline Cystitis & $0.61(0.53-0.69)$ & $0.59(0.50-0.67)$ & $0.55(0.48-0.62)$ \\
\hline Diseases of esophagus & $0.58(0.53-0.62)$ & $0.59(0.55-0.63)$ & $0.54(0.49-0.58)$ \\
\hline Other disorders of synovium, tendon, and bursa & $0.61(0.54-0.69)$ & $0.59(0.52-0.66)$ & $0.61(0.54-0.69)$ \\
\hline GERD & $0.57(0.53-0.62)$ & $0.59(0.55-0.64)$ & $0.55(0.50-0.60)$ \\
\hline Other biliary tract disease & $0.63(0.54-0.71)$ & $0.59(0.51-0.67)$ & $0.59(0.50-0.68)$ \\
\hline Inflammatory and toxic neuropathy & $0.53(0.45-0.61)$ & $0.59(0.52-0.64)$ & $0.43(0.36-0.52)$ \\
\hline Other disorders of liver & $0.60(0.53-0.65)$ & $0.59(0.53-0.64)$ & $0.56(0.49-0.63)$ \\
\hline Other infectious and parasitic diseases & $0.50(0.40-0.59)$ & $0.59(0.49-0.66)$ & $0.57(0.46-0.66)$ \\
\hline Open wounds of head; neck; and trunk & $0.44(0.36-0.54)$ & $0.58(0.48-0.69)$ & $0.47(0.38-0.57)$ \\
\hline Cerebrovascular disease & $0.57(0.52-0.63)$ & $0.58(0.52-0.64)$ & $0.53(0.47-0.60)$ \\
\hline Abnormal electrocardiogram [ECG] [EKG] & $0.49(0.44-0.54)$ & $0.58(0.54-0.64)$ & $0.46(0.41-0.50)$ \\
\hline Generalized anxiety disorder & $0.53(0.46-0.60)$ & $0.58(0.49-0.67)$ & $0.51(0.43-0.59)$ \\
\hline Lymphadenitis & $0.56(0.48-0.62)$ & $0.58(0.51-0.65)$ & $0.53(0.46-0.60)$ \\
\hline Secondary diabetes mellitus & $0.63(0.55-0.72)$ & $0.58(0.50-0.66)$ & $0.67(0.58-0.76)$ \\
\hline Myalgia and myositis unspecified & $0.52(0.46-0.58)$ & $0.58(0.52-0.64)$ & $0.45(0.39-0.50)$ \\
\hline Hypopotassemia & $0.55(0.46-0.63)$ & $0.58(0.50-0.67)$ & $0.51(0.43-0.58)$ \\
\hline Other symptoms/disorders or the urinary system & $0.57(0.53-0.62)$ & $0.58(0.53-0.62)$ & $0.56(0.51-0.61)$ \\
\hline Benign neoplasm of skin & $0.59(0.54-0.64)$ & $0.58(0.52-0.63)$ & $0.57(0.52-0.63)$ \\
\hline Other disorders of circulatory system & $0.44(0.39-0.50)$ & $0.58(0.52-0.65)$ & $0.61(0.54-0.67)$ \\
\hline Dysuria & $0.52(0.45-0.59)$ & $0.58(0.51-0.66)$ & $0.45(0.39-0.53)$ \\
\hline
\end{tabular}


Table S4. Mean (95\% confidence interval) area under the ROC curve for predicting patient diagnoses, grouped into Phecode phenotypes, using the baseline, methylation data, and genotype data. Confidence intervals determined using bootstrapping.

\begin{tabular}{|c|c|c|c|}
\hline Phecode Phenotype & Baseline & Methylation & Genotypes \\
\hline Disorder of skin and subcutaneous tissue NOS & $0.58(0.52-0.64)$ & $0.58(0.52-0.63)$ & $0.53(0.47-0.61)$ \\
\hline Pulmonary heart disease & $0.53(0.47-0.58)$ & $0.58(0.52-0.64)$ & $0.56(0.50-0.63)$ \\
\hline Secondary malignancy of lymph nodes & $0.61(0.52-0.68)$ & $0.58(0.48-0.69)$ & $0.50(0.41-0.60)$ \\
\hline Gastrointestinal hemorrhage & $0.60(0.52-0.67)$ & $0.58(0.51-0.65)$ & $0.56(0.49-0.62)$ \\
\hline Other abnormal blood chemistry & $0.53(0.49-0.58)$ & $0.58(0.53-0.63)$ & $0.49(0.44-0.54)$ \\
\hline Cardiac conduction disorders & $0.54(0.50-0.60)$ & $0.58(0.53-0.62)$ & $0.52(0.47-0.57)$ \\
\hline Nevus, non-neoplastic & $0.58(0.49-0.66)$ & $0.58(0.48-0.66)$ & $0.47(0.39-0.54)$ \\
\hline Congestive heart failure; nonhypertensive & $0.57(0.52-0.62)$ & $0.58(0.52-0.64)$ & $0.53(0.48-0.58)$ \\
\hline Malaise and fatigue & $0.59(0.54-0.64)$ & $0.57(0.53-0.62)$ & $0.56(0.51-0.61)$ \\
\hline Abnormal findings examination of lungs & $0.54(0.49-0.60)$ & $0.57(0.52-0.62)$ & $0.50(0.45-0.55)$ \\
\hline Chronic pain & $0.58(0.54-0.63)$ & $0.57(0.53-0.62)$ & $0.57(0.52-0.62)$ \\
\hline Asthma & $0.57(0.50-0.65)$ & $0.57(0.50-0.64)$ & $0.50(0.42-0.57)$ \\
\hline Hypothyroidism & $0.58(0.51-0.63)$ & $0.57(0.51-0.62)$ & $0.54(0.48-0.60)$ \\
\hline Hearing loss & $0.60(0.55-0.66)$ & $0.57(0.51-0.62)$ & $0.60(0.53-0.66)$ \\
\hline Depression & $0.55(0.49-0.61)$ & $0.57(0.50-0.63)$ & $0.51(0.46-0.56)$ \\
\hline Other peripheral nerve disorders & $0.55(0.49-0.62)$ & $0.57(0.51-0.64)$ & $0.50(0.42-0.58)$ \\
\hline Frequency of urination and polyuria & $0.58(0.52-0.64)$ & $0.57(0.49-0.65)$ & $0.53(0.46-0.62)$ \\
\hline Pericarditis & $0.53(0.44-0.62)$ & $0.57(0.49-0.65)$ & $0.51(0.44-0.59)$ \\
\hline Retention of urine & $0.58(0.52-0.64)$ & $0.57(0.49-0.64)$ & $0.52(0.44-0.59)$ \\
\hline Spinal stenosis & $0.60(0.53-0.67)$ & $0.57(0.50-0.63)$ & $0.56(0.48-0.64)$ \\
\hline Other headache syndromes & $0.45(0.38-0.51)$ & $0.57(0.51-0.63)$ & $0.50(0.45-0.56)$ \\
\hline Other alveolar and parietoalveolar pneumonopathy & $0.62(0.51-0.71)$ & $0.56(0.47-0.66)$ & $0.56(0.46-0.66)$ \\
\hline Spinal stenosis of lumbar region & $0.61(0.54-0.68)$ & $0.56(0.47-0.65)$ & $0.54(0.47-0.63)$ \\
\hline Proteinuria & $0.53(0.45-0.60)$ & $0.56(0.49-0.63)$ & $0.50(0.42-0.59)$ \\
\hline Cardiomyopathy & $0.52(0.46-0.59)$ & $0.56(0.50-0.63)$ & $0.46(0.40-0.53)$ \\
\hline Mitral valve disease & $0.56(0.50-0.63)$ & $0.56(0.50-0.62)$ & $0.58(0.52-0.64)$ \\
\hline Visual disturbances & $0.53(0.44-0.59)$ & $0.56(0.49-0.65)$ & $0.58(0.50-0.65)$ \\
\hline Insomnia & $0.52(0.46-0.59)$ & $0.56(0.49-0.61)$ & $0.53(0.46-0.58)$ \\
\hline Cough & $0.57(0.53-0.62)$ & $0.56(0.52-0.61)$ & $0.56(0.53-0.61)$ \\
\hline Diseases of hair and hair follicles & $0.44(0.35-0.53)$ & $0.56(0.48-0.65)$ & $0.49(0.38-0.58)$ \\
\hline Other symptoms of respiratory system & $0.58(0.54-0.62)$ & $0.56(0.52-0.61)$ & $0.56(0.52-0.60)$ \\
\hline Back pain & $0.58(0.53-0.63)$ & $0.56(0.51-0.60)$ & $0.55(0.51-0.60)$ \\
\hline Neurological disorders & $0.55(0.47-0.61)$ & $0.56(0.48-0.62)$ & $0.54(0.47-0.62)$ \\
\hline Dermatophytosis & $0.59(0.52-0.67)$ & $0.56(0.49-0.62)$ & $0.56(0.48-0.63)$ \\
\hline Alcohol-related disorders & $0.65(0.54-0.76)$ & $0.56(0.45-0.64)$ & $0.58(0.48-0.68)$ \\
\hline Pulmonary congestion and hypostasis & $0.63(0.56-0.70)$ & $0.56(0.48-0.65)$ & $0.59(0.50-0.67)$ \\
\hline Mood disorders & $0.55(0.49-0.60)$ & $0.56(0.50-0.61)$ & $0.49(0.44-0.55)$ \\
\hline Cancer, suspected or other & $0.57(0.51-0.64)$ & $0.56(0.50-0.63)$ & $0.60(0.53-0.66)$ \\
\hline Hypotension NOS & $0.57(0.50-0.62)$ & $0.56(0.50-0.61)$ & $0.52(0.46-0.59)$ \\
\hline Other dyspnea & $0.57(0.52-0.61)$ & $0.56(0.50-0.62)$ & $0.53(0.48-0.58)$ \\
\hline Heart failure with reduced EF [Systolic or comb... & $0.59(0.53-0.66)$ & $0.56(0.48-0.63)$ & $0.56(0.50-0.63)$ \\
\hline Migraine & $0.58(0.49-0.67)$ & $0.56(0.47-0.66)$ & $0.55(0.45-0.66)$ \\
\hline Abnormal findings on exam of gastrointestinal t... & $0.55(0.49-0.65)$ & $0.56(0.47-0.64)$ & $0.49(0.41-0.60)$ \\
\hline
\end{tabular}


Table S4. Mean (95\% confidence interval) area under the ROC curve for predicting patient diagnoses, grouped into Phecode phenotypes, using the baseline, methylation data, and genotype data. Confidence intervals determined using bootstrapping.

\begin{tabular}{|c|c|c|c|}
\hline Phecode Phenotype & Baseline & Methylation & Genotypes \\
\hline Sleep disorders & $0.57(0.51-0.63)$ & $0.56(0.51-0.61)$ & $0.52(0.46-0.57)$ \\
\hline Other local infections of skin and subcutaneous... & $0.57(0.48-0.66)$ & $0.56(0.47-0.64)$ & $0.53(0.43-0.63)$ \\
\hline Anxiety disorder & $0.53(0.47-0.59)$ & $0.56(0.51-0.61)$ & $0.46(0.41-0.52)$ \\
\hline Hemangioma and lymphangioma, any site & $0.56(0.47-0.65)$ & $0.56(0.47-0.66)$ & $0.45(0.36-0.55)$ \\
\hline Hypothyroidism NOS & $0.60(0.55-0.66)$ & $0.55(0.50-0.60)$ & $0.58(0.52-0.63)$ \\
\hline Spondylosis and allied disorders & $0.56(0.50-0.63)$ & $0.55(0.49-0.62)$ & $0.53(0.46-0.59)$ \\
\hline Abdominal hernia & $0.50(0.45-0.55)$ & $0.55(0.49-0.61)$ & $0.46(0.40-0.51)$ \\
\hline Cardiac pacemaker in situ & $0.42(0.34-0.52)$ & $0.55(0.46-0.64)$ & $0.55(0.47-0.64)$ \\
\hline Cerebral ischemia & $0.45(0.36-0.54)$ & $0.55(0.46-0.63)$ & $0.46(0.38-0.53)$ \\
\hline Malignant neoplasm, other & $0.52(0.44-0.59)$ & $0.55(0.48-0.62)$ & $0.46(0.40-0.53)$ \\
\hline Dermatophytosis of nail & $0.59(0.52-0.68)$ & $0.55(0.46-0.65)$ & $0.46(0.37-0.55)$ \\
\hline Cardiac dysrhythmias & $0.56(0.51-0.60)$ & $0.55(0.50-0.59)$ & $0.55(0.50-0.59)$ \\
\hline Cyst of kidney, acquired & $0.53(0.45-0.60)$ & $0.55(0.47-0.62)$ & $0.46(0.38-0.53)$ \\
\hline Impacted cerumen & $0.55(0.46-0.63)$ & $0.55(0.46-0.62)$ & $0.44(0.36-0.51)$ \\
\hline Abnormal heart sounds & $0.56(0.51-0.62)$ & $0.55(0.48-0.62)$ & $0.49(0.43-0.56)$ \\
\hline Neoplasm of uncertain behavior of skin & $0.58(0.52-0.65)$ & $0.55(0.47-0.62)$ & $0.57(0.50-0.63)$ \\
\hline Palpitations & $0.49(0.43-0.55)$ & $0.54(0.47-0.62)$ & $0.51(0.45-0.58)$ \\
\hline Dysphagia & $0.53(0.46-0.59)$ & $0.54(0.46-0.61)$ & $0.55(0.48-0.62)$ \\
\hline Abnormal glucose & $0.55(0.50-0.61)$ & $0.54(0.48-0.59)$ & $0.50(0.45-0.55)$ \\
\hline Functional digestive disorders & $0.49(0.40-0.57)$ & $0.54(0.45-0.63)$ & $0.68(0.58-0.78)$ \\
\hline Syncope and collapse & $0.43(0.36-0.50)$ & $0.54(0.46-0.62)$ & $0.51(0.42-0.60)$ \\
\hline Pruritus and related conditions & $0.47(0.37-0.55)$ & $0.54(0.44-0.63)$ & $0.69(0.61-0.75)$ \\
\hline Allergic rhinitis & $0.56(0.49-0.63)$ & $0.54(0.46-0.61)$ & $0.57(0.52-0.64)$ \\
\hline Esophagitis, GERD and related diseases & $0.56(0.51-0.60)$ & $0.54(0.49-0.59)$ & $0.53(0.49-0.58)$ \\
\hline Cardiac congenital anomalies & $0.46(0.39-0.53)$ & $0.54(0.46-0.61)$ & $0.53(0.44-0.61)$ \\
\hline Major depressive disorder & $0.55(0.48-0.63)$ & $0.54(0.45-0.61)$ & $0.50(0.42-0.57)$ \\
\hline Hemorrhoids & $0.63(0.56-0.71)$ & $0.54(0.46-0.62)$ & $0.55(0.46-0.64)$ \\
\hline Premature beats & $0.55(0.48-0.63)$ & $0.53(0.47-0.59)$ & $0.56(0.48-0.63)$ \\
\hline Ill-defined descriptions and complications of h... & $0.57(0.50-0.62)$ & $0.53(0.46-0.60)$ & $0.49(0.42-0.57)$ \\
\hline Abnormal results of function study of liver & $0.60(0.51-0.69)$ & $0.53(0.44-0.62)$ & $0.54(0.45-0.62)$ \\
\hline Chronic sinusitis & $0.42(0.36-0.49)$ & $0.53(0.46-0.61)$ & $0.46(0.39-0.54)$ \\
\hline Hepatomegaly & $0.51(0.44-0.59)$ & $0.53(0.46-0.60)$ & $0.51(0.42-0.58)$ \\
\hline Diaphragmatic hernia & $0.56(0.49-0.63)$ & $0.53(0.46-0.60)$ & $0.53(0.46-0.60)$ \\
\hline Thoracic or lumbosacral neuritis or radiculitis... & $0.51(0.44-0.59)$ & $0.53(0.47-0.61)$ & $0.48(0.42-0.55)$ \\
\hline Nonspecific chest pain & $0.51(0.46-0.55)$ & $0.53(0.48-0.57)$ & $0.44(0.39-0.49)$ \\
\hline Spondylosis without myelopathy & $0.55(0.48-0.62)$ & $0.53(0.45-0.60)$ & $0.48(0.41-0.55)$ \\
\hline Atopic/contact dermatitis due to other or unspe... & $0.55(0.50-0.62)$ & $0.53(0.47-0.60)$ & $0.56(0.50-0.63)$ \\
\hline Noninfectious gastroenteritis & $0.47(0.39-0.56)$ & $0.53(0.45-0.60)$ & $0.53(0.45-0.60)$ \\
\hline Voice disturbance & $0.53(0.46-0.62)$ & $0.53(0.43-0.61)$ & $0.35(0.26-0.43)$ \\
\hline Chronic kidney disease, Stage I or II & $0.49(0.43-0.56)$ & $0.53(0.45-0.61)$ & $0.41(0.35-0.48)$ \\
\hline Other specified cardiac dysrhythmias & $0.56(0.51-0.62)$ & $0.53(0.46-0.58)$ & $0.52(0.47-0.58)$ \\
\hline Nonrheumatic tricuspid valve disorders & $0.50(0.41-0.57)$ & $0.53(0.45-0.60)$ & $0.45(0.37-0.53)$ \\
\hline Transient cerebral ischemia & $0.45(0.37-0.53)$ & $0.52(0.44-0.60)$ & $0.62(0.54-0.68)$ \\
\hline
\end{tabular}


Table S4. Mean (95\% confidence interval) area under the ROC curve for predicting patient diagnoses, grouped into Phecode phenotypes, using the baseline, methylation data, and genotype data. Confidence intervals determined using bootstrapping.

\begin{tabular}{|c|c|c|c|}
\hline Phecode Phenotype & Baseline & Methylation & Genotypes \\
\hline Intervertebral disc disorders & $0.57(0.51-0.63)$ & $0.52(0.46-0.58)$ & $0.55(0.49-0.60)$ \\
\hline Nonspecific findings on examination of blood & $0.45(0.37-0.51)$ & $0.52(0.45-0.60)$ & $0.53(0.44-0.59)$ \\
\hline Superficial cellulitis and abscess & $0.59(0.52-0.65)$ & $0.52(0.45-0.59)$ & $0.59(0.53-0.66)$ \\
\hline Other abnormal glucose & $0.51(0.46-0.57)$ & $0.52(0.48-0.57)$ & $0.43(0.38-0.48)$ \\
\hline Vitamin D deficiency & $0.56(0.51-0.62)$ & $0.52(0.47-0.58)$ & $0.53(0.47-0.59)$ \\
\hline Rheumatic disease of the heart valves & $0.50(0.42-0.57)$ & $0.52(0.46-0.59)$ & $0.44(0.38-0.51)$ \\
\hline Cancer of urinary organs (incl. kidney and blad... & $0.54(0.46-0.63)$ & $0.52(0.42-0.62)$ & $0.45(0.37-0.53)$ \\
\hline Symptoms and disorders of the joints & $0.57(0.50-0.64)$ & $0.52(0.45-0.60)$ & $0.58(0.49-0.66)$ \\
\hline Empyema and pneumothorax & $0.56(0.48-0.64)$ & $0.52(0.41-0.60)$ & $0.52(0.44-0.60)$ \\
\hline Varicose veins & $0.54(0.47-0.63)$ & $0.52(0.43-0.63)$ & $0.39(0.32-0.47)$ \\
\hline Other disorders of biliary tract & $0.57(0.50-0.66)$ & $0.52(0.42-0.61)$ & $0.49(0.39-0.59)$ \\
\hline Sciatica & $0.47(0.39-0.55)$ & $0.52(0.44-0.59)$ & $0.49(0.41-0.58)$ \\
\hline Abdominal pain & $0.54(0.50-0.59)$ & $0.52(0.47-0.57)$ & $0.55(0.50-0.60)$ \\
\hline Swelling of limb & $0.57(0.51-0.63)$ & $0.52(0.46-0.58)$ & $0.54(0.49-0.61)$ \\
\hline Circulatory disease NEC & $0.47(0.40-0.54)$ & $0.51(0.45-0.57)$ & $0.50(0.44-0.57)$ \\
\hline Sprains and strains & $0.60(0.53-0.67)$ & $0.51(0.43-0.59)$ & $0.52(0.45-0.60)$ \\
\hline Gout & $0.55(0.46-0.63)$ & $0.51(0.43-0.60)$ & $0.56(0.48-0.65)$ \\
\hline Hematuria & $0.56(0.49-0.63)$ & $0.51(0.43-0.57)$ & $0.50(0.43-0.57)$ \\
\hline Arrhythmia (cardiac) NOS & $0.53(0.46-0.59)$ & $0.50(0.43-0.58)$ & $0.54(0.47-0.62)$ \\
\hline Dyschromia and Vitiligo & $0.58(0.51-0.65)$ & $0.50(0.41-0.58)$ & $0.49(0.43-0.57)$ \\
\hline Elevated white blood cell count & $0.52(0.44-0.59)$ & $0.50(0.43-0.58)$ & $0.37(0.30-0.43)$ \\
\hline Encounter for long-term (current) use of antico... & $0.53(0.46-0.61)$ & $0.50(0.45-0.58)$ & $0.50(0.41-0.57)$ \\
\hline Anxiety disorders & $0.54(0.50-0.60)$ & $0.50(0.44-0.55)$ & $0.50(0.44-0.55)$ \\
\hline Allergy/adverse effect of penicillin & $0.53(0.45-0.61)$ & $0.50(0.42-0.60)$ & $0.55(0.45-0.64)$ \\
\hline Diseases of sebaceous glands & $0.49(0.41-0.59)$ & $0.50(0.42-0.58)$ & $0.61(0.54-0.68)$ \\
\hline Rash and other nonspecific skin eruption & $0.54(0.48-0.61)$ & $0.50(0.43-0.56)$ & $0.45(0.39-0.52)$ \\
\hline Acute upper respiratory infections of multiple ... & $0.52(0.45-0.57)$ & $0.49(0.44-0.55)$ & $0.53(0.48-0.59)$ \\
\hline Hypercalcemia & $0.58(0.48-0.67)$ & $0.49(0.41-0.57)$ & $0.46(0.38-0.55)$ \\
\hline Other upper respiratory disease & $0.49(0.43-0.56)$ & $0.49(0.42-0.57)$ & $0.51(0.43-0.58)$ \\
\hline Abnormal serum enzyme levels & $0.48(0.42-0.55)$ & $0.49(0.41-0.56)$ & $0.47(0.39-0.55)$ \\
\hline Paroxysmal ventricular tachycardia & $0.49(0.43-0.58)$ & $0.49(0.41-0.57)$ & $0.43(0.36-0.52)$ \\
\hline Sinoatrial node dysfunction (Bradycardia) & $0.60(0.50-0.69)$ & $0.49(0.40-0.56)$ & $0.64(0.55-0.72)$ \\
\hline Vitamin deficiency & $0.54(0.49-0.59)$ & $0.49(0.43-0.54)$ & $0.51(0.46-0.56)$ \\
\hline Other disorders of arteries and arterioles & $0.46(0.39-0.53)$ & $0.49(0.42-0.57)$ & $0.44(0.38-0.52)$ \\
\hline Gout and other crystal arthropathies & $0.53(0.43-0.61)$ & $0.49(0.41-0.56)$ & $0.57(0.50-0.66)$ \\
\hline Chronic ulcer of skin & $0.49(0.42-0.58)$ & $0.49(0.40-0.57)$ & $0.41(0.33-0.48)$ \\
\hline Swelling, mass, or lump in head and neck [Space... & $0.46(0.38-0.54)$ & $0.49(0.40-0.58)$ & $0.59(0.50-0.66)$ \\
\hline Cardiac and circulatory congenital anomalies & $0.50(0.42-0.56)$ & $0.48(0.42-0.54)$ & $0.40(0.32-0.47)$ \\
\hline Nonspecific abnormal findings on radiological a... & $0.54(0.45-0.63)$ & $0.48(0.40-0.57)$ & $0.50(0.41-0.58)$ \\
\hline Erythematous conditions & $0.57(0.49-0.67)$ & $0.48(0.39-0.58)$ & $0.51(0.41-0.60)$ \\
\hline Poisoning by antibiotics & $0.51(0.43-0.59)$ & $0.48(0.39-0.57)$ & $0.59(0.50-0.68)$ \\
\hline Solitary pulmonary nodule & $0.53(0.45-0.60)$ & $0.48(0.42-0.54)$ & $0.43(0.34-0.50)$ \\
\hline Disorders of refraction and accommodation; blin... & $0.59(0.51-0.70)$ & $0.48(0.39-0.59)$ & $0.49(0.39-0.60)$ \\
\hline
\end{tabular}


Table S4. Mean (95\% confidence interval) area under the ROC curve for predicting patient diagnoses, grouped into Phecode phenotypes, using the baseline, methylation data, and genotype data. Confidence intervals determined using bootstrapping.

\begin{tabular}{llll}
\hline Phecode Phenotype & Baseline & Methylation & Genotypes \\
\hline Disturbance of skin sensation & $0.50(0.43-0.58)$ & $0.47(0.40-0.56)$ & $0.49(0.41-0.58)$ \\
Paroxysmal tachycardia, unspecified & $0.41(0.34-0.49)$ & $0.47(0.38-0.55)$ & $0.40(0.32-0.49)$ \\
Disease of tricuspid valve & $0.45(0.38-0.54)$ & $0.47(0.38-0.55)$ & $0.55(0.48-0.64)$ \\
Other symptoms involving abdomen and pelvis & $0.56(0.47-0.62)$ & $0.47(0.39-0.55)$ & $0.55(0.47-0.61)$ \\
Other specified diseases of hair and hair folli... & $0.39(0.31-0.47)$ & $0.47(0.38-0.56)$ & $0.52(0.43-0.61)$ \\
Other dyschromia & $0.56(0.48-0.63)$ & $0.46(0.38-0.54)$ & $0.50(0.42-0.58)$ \\
Valvular heart disease/ heart chambers & $0.48(0.41-0.56)$ & $0.45(0.37-0.54)$ & $0.54(0.45-0.62)$ \\
Heart transplant/surgery & $0.49(0.40-0.58)$ & $0.45(0.37-0.53)$ & $0.48(0.41-0.55)$ \\
Calculus of kidney & $0.54(0.47-0.61)$ & $0.45(0.37-0.52)$ & $0.52(0.45-0.58)$ \\
Acute pharyngitis & $0.43(0.35-0.51)$ & $0.45(0.37-0.53)$ & $0.42(0.35-0.48)$ \\
Acute bronchitis and bronchiolitis & $0.52(0.44-0.61)$ & $0.44(0.36-0.55)$ & $0.55(0.45-0.63)$ \\
Diseases of pancreas & $0.51(0.42-0.58)$ & $0.44(0.37-0.53)$ & $0.43(0.36-0.49)$ \\
Paroxysmal supraventricular tachycardia & $0.49(0.42-0.57)$ & $0.43(0.34-0.51)$ & $0.57(0.48-0.65)$ \\
Opiates and related narcotics causing adverse e... & $0.59(0.50-0.68)$ & $0.43(0.35-0.51)$ & $0.45(0.36-0.54)$ \\
Other diseases of respiratory system, not elsew... & $0.51(0.40-0.60)$ & $0.42(0.34-0.50)$ & $0.49(0.40-0.57)$ \\
Cervicalgia & $0.51(0.44-0.59)$ & $0.42(0.36-0.51)$ & $0.52(0.44-0.59)$ \\
Complications of cardiac/vascular device, impla... & $0.54(0.47-0.61)$ & $0.42(0.32-0.53)$ & $0.51(0.42-0.59)$ \\
Other hypertrophic and atrophic conditions of skin & $0.55(0.47-0.63)$ & $0.40(0.32-0.47)$ & $0.42(0.36-0.50)$ \\
\hline
\end{tabular}


Table S5. The imputation accuracy, p-value and number of CpGs selected for significantly imputed MRS that also significantly improved over the baseline model.

\begin{tabular}{|c|c|c|c|c|}
\hline Pharmaceutical Subclass & Medication GPI & AUC & p-value & Number of CpGs \\
\hline Phosphate Binder Agents & 5280 & 0.876 & $1.106 \mathrm{E}-50$ & 2715 \\
\hline Hematopoietic Growth Factors & 8240 & 0.840 & $1.747 \mathrm{E}-45$ & 996 \\
\hline Immunosuppressive Agents & 9940 & 0.828 & $9.443 \mathrm{E}-41$ & 2870 \\
\hline CMV Agents & 1220 & 0.905 & $1.720 \mathrm{E}-38$ & 1223 \\
\hline Osmotic Diuretics & 3740 & 0.848 & $6.372 \mathrm{E}-34$ & 510 \\
\hline B-Complex w/ Folic Acid & 7813 & 0.836 & $3.442 \mathrm{E}-31$ & 2593 \\
\hline Metabolic Modifiers & 3090 & 0.813 & $1.213 \mathrm{E}-28$ & 2200 \\
\hline Prostatic Hypertrophy Agents & 5685 & 0.779 & $2.411 \mathrm{E}-25$ & 2586 \\
\hline Plasma Proteins & 8540 & 0.739 & $4.379 \mathrm{E}-24$ & 3353 \\
\hline Proton Pump Inhibitors & 4927 & 0.693 & $3.500 \mathrm{E}-23$ & 1097 \\
\hline Anti-infectives - Throat & 8810 & 0.765 & $2.443 \mathrm{E}-20$ & 453 \\
\hline Imidazole-Related Antifungals & 1140 & 0.720 & $2.249 \mathrm{E}-17$ & 2507 \\
\hline Vasodilators & 3640 & 0.708 & $6.753 \mathrm{E}-17$ & 654 \\
\hline Glucocorticosteroids & 2210 & 0.663 & $1.354 \mathrm{E}-16$ & 4508 \\
\hline Loop Diuretics & 3720 & 0.674 & $5.020 \mathrm{E}-16$ & 4529 \\
\hline Anti-infective Misc. - Combinations & 1699 & 0.665 & $7.711 \mathrm{E}-16$ & 3503 \\
\hline Analgesics Other & 6420 & 0.651 & $1.084 \mathrm{E}-15$ & 833 \\
\hline Antihistamines - Ethanolamines & 4120 & 0.660 & $1.056 \mathrm{E}-14$ & 649 \\
\hline Laxatives - Miscellaneous & 4660 & 0.648 & $4.984 \mathrm{E}-14$ & 4203 \\
\hline Anti-infective Agents - Misc. & 1600 & 0.674 & $9.525 \mathrm{E}-14$ & 657 \\
\hline 5-HT3 Receptor Antagonists & 5025 & 0.653 & $1.049 \mathrm{E}-13$ & 4570 \\
\hline Fluoroquinolones & 500 & 0.658 & $1.885 \mathrm{E}-13$ & 510 \\
\hline Benzodiazepines & 5710 & 0.658 & $1.957 \mathrm{E}-13$ & 4482 \\
\hline Potassium Removing Agents & 9945 & 0.736 & $2.568 \mathrm{E}-13$ & 2048 \\
\hline Cephalosporins - 3rd Generation & 230 & 0.665 & $4.926 \mathrm{E}-13$ & 2379 \\
\hline Stimulant Laxatives & 4620 & 0.642 & $1.014 \mathrm{E}-12$ & 3949 \\
\hline Calcium Channel Blockers & 3400 & 0.638 & $3.149 \mathrm{E}-12$ & 4658 \\
\hline Insulin & 2710 & 0.654 & $3.947 \mathrm{E}-12$ & 3385 \\
\hline Carbohydrates & 8010 & 0.652 & $5.207 \mathrm{E}-12$ & 4246 \\
\hline Thrombolytic Enzymes & 8560 & 0.747 & $5.481 \mathrm{E}-12$ & 1504 \\
\hline Parenteral Therapy Supplies & 9705 & 0.709 & $1.580 \mathrm{E}-11$ & 554 \\
\hline Local Anesthetic Combinations & 6999 & 0.655 & $2.178 \mathrm{E}-11$ & 3235 \\
\hline Heparins And Heparinoid-Like Agents & 8310 & 0.637 & $2.486 \mathrm{E}-11$ & 607 \\
\hline Electrolyte Mixtures & 7999 & 0.634 & $4.224 \mathrm{E}-11$ & 591 \\
\hline Potassium & 7970 & 0.643 & $4.564 \mathrm{E}-11$ & 870 \\
\hline Calcium & 7910 & 0.659 & $6.164 \mathrm{E}-11$ & 3126 \\
\hline Alpha-Beta Blockers & 3330 & 0.647 & $7.206 \mathrm{E}-11$ & 4029 \\
\hline Beta Blockers Cardio-Selective & 3320 & 0.618 & $8.699 \mathrm{E}-11$ & 5159 \\
\hline Glycopeptides & 1628 & 0.639 & $2.870 \mathrm{E}-10$ & 2999 \\
\hline Saline Laxatives & 4610 & 0.643 & $3.819 \mathrm{E}-10$ & 3509 \\
\hline Magnesium & 7940 & 0.636 & $1.011 \mathrm{E}-09$ & 3643 \\
\hline Diagnostic Radiopharmaceuticals & 9435 & 0.630 & $4.355 \mathrm{E}-09$ & 652 \\
\hline Antiseptics - Mouth/Throat & 8815 & 0.673 & $5.057 \mathrm{E}-09$ & 2134 \\
\hline Vasopressors & 3800 & 0.624 & $9.485 \mathrm{E}-09$ & 4378 \\
\hline
\end{tabular}


Table S5. The imputation accuracy, p-value and number of CpGs selected for significantly imputed MRS that also significantly improved over the baseline model.

\begin{tabular}{llrrr}
\hline Pharmaceutical Subclass & Medication GPI & AUC & p-value & Number of CpGs \\
\hline Phenothiazines & 5920 & 0.616 & $1.141 \mathrm{E}-07$ & 353 \\
Antiperistaltic Agents & 4710 & 0.655 & $3.923 \mathrm{E}-07$ & 468 \\
Alternative Medicine - C's & 9509 & 0.658 & $2.111 \mathrm{E}-06$ & 135 \\
Liquid Vehicles & 9840 & 0.655 & $2.814 \mathrm{E}-06$ & 1372 \\
Antiflatulents & 5220 & 0.624 & $5.187 \mathrm{E}-06$ & 1750 \\
Alternative Medicine - M's & 9539 & 0.652 & $6.305 \mathrm{E}-06$ & 1792 \\
Misc. Nutritional Substances & 8050 & 0.616 & $1.029 \mathrm{E}-05$ & 2702 \\
Gallstone Solubilizing Agents & 5210 & 0.724 & $1.071 \mathrm{E}-05$ & 1177 \\
Thiazides and Thiazide-Like Diuretics & 3760 & 0.629 & $1.291 \mathrm{E}-05$ & 73 \\
Corticosteroids - Topical & 9055 & 0.575 & $1.354 \mathrm{E}-04$ & 44 \\
\hline \hline
\end{tabular}




\begin{tabular}{lrrr}
\hline Lab & $R^{2}$ & p-value & Number of CpGs \\
\hline Creatinine & 0.457 & $1.266 \mathrm{E}-95$ & 32364 \\
Urea Nitrogen & 0.435 & $2.502 \mathrm{E}-87$ & 4218 \\
Absolute Eos Count & 0.352 & $3.960 \mathrm{E}-51$ & 3602 \\
Hemoglobin & 0.284 & $3.024 \mathrm{E}-50$ & 25995 \\
Hematocrit & 0.246 & $1.144 \mathrm{E}-42$ & 1827 \\
Neutrophil Percent, Auto & 0.264 & $1.139 \mathrm{E}-36$ & 897 \\
Mean Corpuscular Hemoglobin & 0.208 & $7.037 \mathrm{E}-35$ & 33151 \\
Mean Corpuscular Volume & 0.183 & $1.439 \mathrm{E}-30$ & 60383 \\
Platelet Count, Auto & 0.168 & $1.956 \mathrm{E}-28$ & 15362 \\
Chloride & 0.141 & $1.493 \mathrm{E}-24$ & 44943 \\
Albumin & 0.143 & $6.472 \mathrm{E}-22$ & 6020 \\
Absolute Immature Gran Count & 0.155 & $1.545 \mathrm{E}-20$ & 1705 \\
White Blood Cell Count & 0.120 & $3.405 \mathrm{E}-20$ & 29995 \\
Absolute Neut Count & 0.149 & $4.974 \mathrm{E}-20$ & 19924 \\
Sodium & 0.115 & $5.060 \mathrm{E}-20$ & 35598 \\
Absolute Lymphocyte Count & 0.128 & $2.920 \mathrm{E}-17$ & 10575 \\
Absolute Mono Count & 0.121 & $2.579 \mathrm{E}-16$ & 3675 \\
Neutrophils Abs (Prelim) & 0.116 & $1.263 \mathrm{E}-15$ & 18192 \\
Glucose & 0.075 & $4.033 \mathrm{E}-13$ & 973 \\
Absolute Baso Count & 0.086 & $7.020 \mathrm{E}-12$ & 566 \\
Hgb A1c - Hplc & 0.125 & $2.588 \mathrm{E}-11$ & 902 \\
Ferritin & 0.122 & $1.331 \mathrm{E}-07$ & 40620 \\
Iron Binding Capacity & 0.104 & $8.842 \mathrm{E}-07$ & 7121 \\
Qrs Duration & 0.052 & $1.722 \mathrm{E}-06$ & 4731 \\
Anion Gap & 0.027 & $1.729 \mathrm{E}-05$ & 29869 \\
Alanine Aminotransferase & 0.028 & $2.801 \mathrm{E}-05$ & 546 \\
Cholesterol, Hdl & 0.054 & $4.612 \mathrm{E}-05$ & 5303 \\
Ventricular Rate & 0.033 & $1.499 \mathrm{E}-04$ & \\
Bilirubin,Total & 0.022 & $3.094 \mathrm{E}-04$ & \\
\hline
\end{tabular}

Table S6. The imputation accuracy, p-value and number of CpGs selected for significantly imputed MRS that also significantly improved over the baseline model. 
Table S7. The imputation accuracy, p-value and number of CpGs selected for significantly imputed MRS that also significantly improved over the baseline model.

\begin{tabular}{|c|c|c|c|c|}
\hline Phenotype & Phecode & AUC & p-value & Number of CpGs \\
\hline End stage renal disease & 585.32 & 0.898 & $5.459 \mathrm{E}-72$ & 2790 \\
\hline Chronic renal failure $[\mathrm{CKD}]$ & 585.3 & 0.820 & $1.814 \mathrm{E}-56$ & 4357 \\
\hline Renal dialysis & 585.31 & 0.880 & $9.402 \mathrm{E}-56$ & 2654 \\
\hline Renal failure & 585.0 & 0.798 & $6.810 \mathrm{E}-51$ & 4836 \\
\hline Hypertensive chronic kidney disease & 401.22 & 0.801 & $1.446 \mathrm{E}-42$ & 3851 \\
\hline Immunity deficiency & 279.1 & 0.821 & $7.942 \mathrm{E}-33$ & 624 \\
\hline Anemia of chronic disease & 285.2 & 0.789 & $1.395 \mathrm{E}-32$ & 3391 \\
\hline Disorders involving the immune mechanism & 279.0 & 0.799 & $1.072 \mathrm{E}-30$ & 2681 \\
\hline Anemia in chronic kidney disease & 285.21 & 0.813 & $5.977 \mathrm{E}-27$ & 537 \\
\hline Kidney replaced by transpant & 587.0 & 0.813 & $8.000 \mathrm{E}-27$ & 605 \\
\hline Morbid obesity & 278.11 & 0.847 & $2.416 \mathrm{E}-26$ & 158 \\
\hline Cirrhosis of liver without mention of alcohol & 571.51 & 0.856 & $2.588 \mathrm{E}-25$ & 441 \\
\hline Essential hypertension & 401.1 & 0.701 & $1.007 \mathrm{E}-22$ & 4666 \\
\hline Disorders resulting from impaired renal function & 588.0 & 0.796 & $1.982 \mathrm{E}-20$ & 1944 \\
\hline Decreased white blood cell count & 288.1 & 0.811 & $7.196 \mathrm{E}-20$ & 1716 \\
\hline Neutropenia & 288.11 & 0.836 & $1.106 \mathrm{E}-19$ & 1137 \\
\hline Type 2 diabetes & 250.2 & 0.698 & $8.981 \mathrm{E}-19$ & 500 \\
\hline Hypertensive heart and/or renal disease & 401.2 & 0.723 & $1.408 \mathrm{E}-17$ & 2804 \\
\hline Acute renal failure & 585.1 & 0.704 & $1.739 \mathrm{E}-17$ & 551 \\
\hline Hypertension & 401.0 & 0.674 & $1.852 \mathrm{E}-17$ & 563 \\
\hline Secondary hyperparathyroidism (of renal origin) & 588.2 & 0.763 & $1.011 \mathrm{E}-15$ & 1942 \\
\hline Poisoning by primarily systemic agents & 963.0 & 0.818 & $1.029 \mathrm{E}-14$ & 1022 \\
\hline Other anemias & 285.0 & 0.667 & $1.134 \mathrm{E}-14$ & 4294 \\
\hline Other disorders of the kidney and ureters & 586.0 & 0.685 & $3.876 \mathrm{E}-14$ & 3263 \\
\hline Hyperpotassemia & 276.13 & 0.706 & $1.047 \mathrm{E}-13$ & 2642 \\
\hline Fluid overload & 276.6 & 0.756 & $3.137 \mathrm{E}-13$ & 1667 \\
\hline Acid-base balance disorder & 276.4 & 0.733 & $3.331 \mathrm{E}-13$ & 1863 \\
\hline Respiratory failure, insufficiency, arrest & 509.0 & 0.729 & $1.338 \mathrm{E}-11$ & 491 \\
\hline Portal hypertension & 571.81 & 0.803 & $2.149 \mathrm{E}-11$ & 1347 \\
\hline Chronic liver disease and cirrhosis & 571.0 & 0.707 & $6.362 \mathrm{E}-11$ & 429 \\
\hline $\begin{array}{l}\text { Disorders of fluid, electrolyte, and acid-base bal- } \\
\text { ance }\end{array}$ & 276.0 & 0.664 & $7.581 \mathrm{E}-11$ & 3827 \\
\hline Abnormal involuntary movements & 350.1 & 0.757 & $7.668 \mathrm{E}-10$ & 284 \\
\hline Liver abscess and sequelae of chronic liver disease & 571.8 & 0.782 & $9.916 \mathrm{E}-10$ & 1420 \\
\hline Purpura and other hemorrhagic conditions & 287.0 & 0.714 & $1.059 \mathrm{E}-09$ & 1747 \\
\hline Liver replaced by transplant & 573.2 & 0.764 & $1.119 \mathrm{E}-09$ & 317 \\
\hline Thrombocytopenia & 287.3 & 0.697 & $1.367 \mathrm{E}-09$ & 433 \\
\hline Splenomegaly & 579.2 & 0.705 & $6.780 \mathrm{E}-09$ & 350 \\
\hline Coagulation defects & 286.0 & 0.738 & $1.068 \mathrm{E}-08$ & 453 \\
\hline Altered mental status & 292.4 & 0.722 & $1.817 \mathrm{E}-08$ & 1054 \\
\hline $\begin{array}{l}\text { Nephritis and nephropathy without mention of } \\
\text { glomerulonephritis }\end{array}$ & 580.3 & 0.679 & $2.788 \mathrm{E}-08$ & 1640 \\
\hline Type 2 diabetes with renal manifestations & 250.22 & 0.680 & $3.467 \mathrm{E}-08$ & 1716 \\
\hline Nephritis; nephrosis; renal sclerosis & 580.0 & 0.664 & $4.350 \mathrm{E}-08$ & 2296 \\
\hline
\end{tabular}


Table S7. The imputation accuracy, p-value and number of CpGs selected for significantly imputed MRS that also significantly improved over the baseline model.

\begin{tabular}{llrrr}
\hline Phenotype & Phecode & AUC & p-value & Number of CpGs \\
\hline Nausea and vomiting & 789.0 & 0.650 & $7.959 \mathrm{E}-08$ & 2725 \\
Antineoplastic and immunosuppressive drugsad- & 963.1 & 0.786 & $3.432 \mathrm{E}-07$ & 290 \\
verse effects & & & & \\
Acute pain & 338.1 & 0.649 & $4.095 \mathrm{E}-07$ & 2306 \\
Cardiomegaly & 416.0 & 0.622 & $4.106 \mathrm{E}-07$ & 3672 \\
Senile cataract & 366.2 & 0.657 & $4.269 \mathrm{E}-07$ & 1875 \\
Other disorders of metabolism & 277.0 & 0.704 & $5.053 \mathrm{E}-07$ & 1362 \\
Chronic Kidney Disease, Stage III & 585.33 & 0.651 & $1.493 \mathrm{E}-06$ & 515 \\
Renal failure NOS & 585.2 & 0.692 & $1.697 \mathrm{E}-06$ & 1197 \\
Chronic Kidney Disease, Stage IV & 585.34 & 0.675 & $3.079 \mathrm{E}-06$ & 1956 \\
Atherosclerosis & 440.0 & 0.660 & $8.130 \mathrm{E}-06$ & 329 \\
Heart failure NOS & 428.2 & 0.623 & $1.243 \mathrm{E}-05$ & 2759 \\
Poisoning by hormones and synthetic substitutes & 962.0 & 0.690 & $1.919 \mathrm{E}-05$ & 1061 \\
Iron deficiency anemia secondary to blood loss & 280.2 & 0.667 & $3.463 \mathrm{E}-05$ & 269 \\
(chronic) & & & \\
Other hypertrophic and atrophic conditions of & 701.0 & 0.632 & $5.942 \mathrm{E}-05$ & 951 \\
skin & & & & \\
\hline \hline
\end{tabular}


Table S8. Number of samples with reported usage of medications in the pharmaceutical subclasses. Pharmaceutical subclasses are sorted by number of samples.

\begin{tabular}{|c|c|}
\hline Pharmaceutical Subclass & Number of Samples (Percent) \\
\hline Sodium & $699(80.9 \%)$ \\
\hline Opioid Agonists & $639(74.0 \%)$ \\
\hline Local Anesthetics - Amides & $589(68.2 \%)$ \\
\hline Non-Barbiturate Hypnotics & $584(67.6 \%)$ \\
\hline 5-HT3 Receptor Antagonists & $549(63.5 \%)$ \\
\hline Analgesics Other & $544(63.0 \%)$ \\
\hline Radiographic Contrast Media & $535(61.9 \%)$ \\
\hline Anesthetics - Misc. & $507(58.7 \%)$ \\
\hline Glucocorticosteroids & $499(57.8 \%)$ \\
\hline Salicylates & $459(53.1 \%)$ \\
\hline Heparins And Heparinoid-Like Agents & $459(53.1 \%)$ \\
\hline Opioid Combinations & $458(53.0 \%)$ \\
\hline HMG CoA Reductase Inhibitors & $456(52.8 \%)$ \\
\hline Proton Pump Inhibitors & $443(51.3 \%)$ \\
\hline Oil Soluble Vitamins & $434(50.2 \%)$ \\
\hline Vasopressors & $421(48.7 \%)$ \\
\hline Surfactant Laxatives & $398(46.1 \%)$ \\
\hline Electrolyte Mixtures & $390(45.1 \%)$ \\
\hline Antiarrhythmics Type I-B & $383(44.3 \%)$ \\
\hline Beta Blockers Cardio-Selective & $383(44.3 \%)$ \\
\hline Cephalosporins - 1st Generation & $369(42.7 \%)$ \\
\hline Calcium Channel Blockers & $367(42.5 \%)$ \\
\hline Loop Diuretics & $346(40.0 \%)$ \\
\hline Miscellaneous Contrast Media & $341(39.5 \%)$ \\
\hline Nondepolarizing Muscle Relaxants & $336(38.9 \%)$ \\
\hline Fluoroquinolones & $327(37.8 \%)$ \\
\hline Stimulant Laxatives & $326(37.7 \%)$ \\
\hline Nonsteroidal Anti-inflammatory Agents (NSAIDs) & $313(36.2 \%)$ \\
\hline Sympathomimetics & $308(35.6 \%)$ \\
\hline Antihistamines - Ethanolamines & $301(34.8 \%)$ \\
\hline Laxatives - Miscellaneous & $293(33.9 \%)$ \\
\hline Magnesium & $290(33.6 \%)$ \\
\hline Local Anesthetics - Topical & $280(32.4 \%)$ \\
\hline Potassium & $277(32.1 \%)$ \\
\hline Insulin & $269(31.1 \%)$ \\
\hline Benzodiazepines & $265(30.7 \%)$ \\
\hline Diagnostic Radiopharmaceuticals & $264(30.6 \%)$ \\
\hline Anticonvulsants - Misc. & $260(30.1 \%)$ \\
\hline Carbohydrates & $252(29.2 \%)$ \\
\hline Saline Laxatives & $250(28.9 \%)$ \\
\hline Antispasmodics & $250(28.9 \%)$ \\
\hline H-2 Antagonists & $232(26.9 \%)$ \\
\hline Angiotensin II Receptor Antagonists & $231(26.7 \%)$ \\
\hline ACE Inhibitors & $225(26.0 \%)$ \\
\hline Penicillin Combinations & $219(25.3 \%)$ \\
\hline Cephalosporins - 3rd Generation & $217(25.1 \%)$ \\
\hline
\end{tabular}


Nitrates

Glycopeptides

Alpha-Beta Blockers

Calcium

Multivitamins

Local Anesthetic Combinations

Anti-infective Misc. - Combinations

Anti-infective Agents - Misc.

Plasma Proteins

Diagnostic Drugs

Water Soluble Vitamins

Phenothiazines

Gastrointestinal Stimulants

Corticosteroids - Topical

Central Muscle Relaxants

Viral Vaccines

Iron

Vasodilators

Antibiotics - Topical

Hematopoietic Growth Factors

Azithromycin

Antacids - Calcium Salts

Antimyasthenic/Cholinergic Agents

Nasal Steroids

Selective Serotonin Reuptake Inhibitors (SSRIs)

Thiazides and Thiazide-Like Diuretics

Misc. Nutritional Substances

Opioid Antagonists

Platelet Aggregation Inhibitors

Thyroid Hormones

Antifungals - Topical

Bacterial Vaccines

Immunosuppressive Agents

Phosphate Binder Agents

Serotonin Modulators

Laxative Combinations

Biguanides

Depolarizing Muscle Relaxants

Genitourinary Irrigants

Prostatic Hypertrophy Agents

Bronchodilators - Anticholinergics

Antiflatulents

Antacid Combinations

Aminopenicillins

Imidazole-Related Antifungals

Diagnostic Tests

Cobalamins

Folic Acid/Folates

B-Complex w/ Folic Acid

Antihistamines - Non-Sedating

Anesthetics Topical Oral
$215(24.9 \%)$

$213(24.7 \%)$

$210(24.3 \%)$

$207(24.0 \%)$

$207(24.0 \%)$

$200(23.1 \%)$

$198(22.9 \%)$

$193(22.3 \%)$

$190(22.0 \%)$

$189(21.9 \%)$

$188(21.8 \%)$

$184(21.3 \%)$

$182(21.1 \%)$

$182(21.1 \%)$

$181(20.9 \%)$

$175(20.3 \%)$

$170(19.7 \%)$

$168(19.4 \%)$

$166(19.2 \%)$

$165(19.1 \%)$

$164(19.0 \%)$

$164(19.0 \%)$

$158(18.3 \%)$

$158(18.3 \%)$

$157(18.2 \%)$

$157(18.2 \%)$

$155(17.9 \%)$

$155(17.9 \%)$

$154(17.8 \%)$

$149(17.2 \%)$

$149(17.2 \%)$

$144(16.7 \%)$

$142(16.4 \%)$

$140(16.2 \%)$

$136(15.7 \%)$

$136(15.7 \%)$

$135(15.6 \%)$

$135(15.6 \%)$

$134(15.5 \%)$

$134(15.5 \%)$

$131(15.2 \%)$

$130(15.0 \%)$

$127(14.7 \%)$

$126(14.6 \%)$

$125(14.5 \%)$

$120(13.9 \%)$

$118(13.7 \%)$

$116(13.4 \%)$

$116(13.4 \%)$

$113(13.1 \%)$

$108(12.5 \%)$ 
Diabetic Supplies

Osmotic Diuretics

Tetracyclines

Multiple Vitamins w/ Minerals

Ophthalmic Anti-infectives

Metabolic Modifiers

Potassium Removing Agents

Potassium Sparing Diuretics

Hemostatics - Topical

Ophthalmics - Misc.

Gout Agents

Alternative Medicine - M's

Parenteral Therapy Supplies

Cough/Cold/Allergy Combinations

Antiseptics - Mouth/Throat

Direct Factor Xa Inhibitors

Anti-infectives - Throat

Anti-inflammatory Agents - Topical

Coumarin Anticoagulants

Posterior Pituitary Hormones

Antidotes and Specific Antagonists

Antiadrenergic Antihypertensives

Ophthalmic Steroids

Antitussives

Lincosamides

Dibenzapines

Bone Density Regulators

Antianxiety Agents - Misc.

Phosphate

Antiemetics - Anticholinergic

Antiperistaltic Agents

Herpes Agents

Bicarbonates

Liquid Vehicles

Antiarrhythmics Type III

Artificial Tears and Lubricants

Antidiarrheal/Probiotic Agents - Misc.

Toxoid Combinations

Urinary Antispasmodic - Antimuscarinics (Antich...

Lozenges

CMV Agents

Thrombolytic Enzymes

Impotence Agents

Alternative Medicine - C's

Sulfonylureas

Antihypertensive Combinations

Specialty Vitamins Products

Aminoglycosides

Cephalosporins - 2nd Generation

Alkalinizers

Opioid Partial Agonists
$107(12.4 \%)$

$106(12.3 \%)$

$105(12.2 \%)$

$105(12.2 \%)$

$104(12.0 \%)$

$102(11.8 \%)$

$102(11.8 \%)$

$101(11.7 \%)$

$101(11.7 \%)$

$101(11.7 \%)$

$100(11.6 \%)$

$99(11.5 \%)$

$99(11.5 \%)$

$99(11.5 \%)$

$98(11.3 \%)$

$97(11.2 \%)$

$94(10.9 \%)$

$93(10.8 \%)$

$92(10.6 \%)$

91 (10.5\%)

$90(10.4 \%)$

$90(10.4 \%)$

$90(10.4 \%)$

$88(10.2 \%)$

$84(9.7 \%)$

$83(9.6 \%)$

$81(9.4 \%)$

$80(9.3 \%)$

$78(9.0 \%)$

$77(8.9 \%)$

$76(8.8 \%)$

$76(8.8 \%)$

$75(8.7 \%)$

$72(8.3 \%)$

$72(8.3 \%)$

$71(8.2 \%)$

$71(8.2 \%)$

$70(8.1 \%)$

$67(7.8 \%)$

$67(7.8 \%)$

$66(7.6 \%)$

$66(7.6 \%)$

$65(7.5 \%)$

$64(7.4 \%)$

$63(7.3 \%)$

$63(7.3 \%)$

$63(7.3 \%)$

$61(7.1 \%)$

$60(6.9 \%)$

$59(6.8 \%)$

$73(6.8 \%)$ 
Urinary Anti-infectives

Irrigation Solutions

Influenza Agents

Expectorants

Beta Blockers Non-Selective

Tricyclic Agents

Serotonin-Norepinephrine Reuptake Inhibitors (S...

Cephalosporins - 4th Generation

Antihistamines-Topical

Antacids - Bicarbonate

Bulk Laxatives

Alpha-2 Receptor Antagonists (Tetracyclics)

Ophthalmic Local Anesthetics

Hemostatics - Systemic

Zinc

Dipeptidyl Peptidase-4 (DPP-4) Inhibitors

Gallstone Solubilizing Agents

Cycloplegic Mydriatics

Protamine

Butyrophenones

Antidepressants - Misc.

Mucolytics

Leukotriene Modulators

B-Complex Vitamins

Acne Products
$58(6.7 \%)$

$58(6.7 \%)$

$57(6.6 \%)$

$57(6.6 \%)$

$56(6.5 \%)$

$56(6.5 \%)$

$56(6.5 \%)$

$55(6.4 \%)$

$55(6.4 \%)$

$54(6.2 \%)$

$53(6.1 \%)$

$52(6.0 \%)$

$49(5.7 \%)$

$49(5.7 \%)$

$48(5.6 \%)$

$47(5.4 \%)$

$47(5.4 \%)$

$47(5.4 \%)$

$58(5.4 \%)$

$46(5.3 \%)$

$45(5.2 \%)$

$45(5.2 \%)$

$44(5.1 \%)$

$44(5.1 \%)$

$44(5.1 \%)$ 
Table S9. Medications used in each pharmaceutical subclass

\begin{tabular}{|c|c|}
\hline Pharmaceutical Subclass & Drug Name \\
\hline ALKALINIZERS & BICITRA \\
\hline ALKALINIZERS & CITRIC \\
\hline ALKALINIZERS & CYTRA-2 \\
\hline ALKALINIZERS & CYTRA-3 \\
\hline ALKALINIZERS & POT \\
\hline ALKALINIZERS & POTASSIUM \\
\hline ANTI-INFECTIVES - THROAT & CLOTRIMAZOLE \\
\hline ANTI-INFECTIVES - THROAT & MICONAZOLE \\
\hline ANTI-INFECTIVES - THROAT & NYSTATIN \\
\hline B-COMPLEX W/ FOLIC ACID & $\mathrm{B}$ \\
\hline B-COMPLEX W/ FOLIC ACID & B-COMPLEX \\
\hline B-COMPLEX W/ FOLIC ACID & DIALYVITE \\
\hline B-COMPLEX W/ FOLIC ACID & FULL \\
\hline B-COMPLEX W/ FOLIC ACID & NEPHRO-VITE \\
\hline B-COMPLEX W/ FOLIC ACID & NEPHROCAPS \\
\hline B-COMPLEX W/ FOLIC ACID & RENA-VITE \\
\hline B-COMPLEX W/ FOLIC ACID & RENAL \\
\hline B-COMPLEX W/ FOLIC ACID & RENAL-VITE \\
\hline B-COMPLEX W/ FOLIC ACID & VOL-CARE \\
\hline B-COMPLEX W/ FOLIC ACID & VP-VITE \\
\hline BIGUANIDES & METFORMIN \\
\hline CALCIUM CHANNEL BLOCKERS & ADALAT \\
\hline CALCIUM CHANNEL BLOCKERS & AFEDITAB \\
\hline CALCIUM CHANNEL BLOCKERS & AMLODIPINE \\
\hline CALCIUM CHANNEL BLOCKERS & CARTIA \\
\hline CALCIUM CHANNEL BLOCKERS & DILT-XR \\
\hline CALCIUM CHANNEL BLOCKERS & DILTIAZEM \\
\hline CALCIUM CHANNEL BLOCKERS & FELODIPINE \\
\hline CALCIUM CHANNEL BLOCKERS & ISRADIPINE \\
\hline CALCIUM CHANNEL BLOCKERS & NICARDIPINE \\
\hline CALCIUM CHANNEL BLOCKERS & NIFEDICAL \\
\hline CALCIUM CHANNEL BLOCKERS & NIFEDIPINE \\
\hline CALCIUM CHANNEL BLOCKERS & NIMODIPINE \\
\hline CALCIUM CHANNEL BLOCKERS & NORVASC \\
\hline CALCIUM CHANNEL BLOCKERS & VERAPAMIL \\
\hline CMV AGENTS & VALCYTE \\
\hline CMV AGENTS & VALGANCICLOVIR \\
\hline DIBENZAPINES & OLANZAPINE \\
\hline DIBENZAPINES & QUETIAPINE \\
\hline DIBENZAPINES & ZYPREXA \\
\hline HEMATOPOIETIC GROWTH FACTORS & ARANESP \\
\hline HEMATOPOIETIC GROWTH FACTORS & DARBEPOETIN \\
\hline HEMATOPOIETIC GROWTH FACTORS & EPOETIN \\
\hline HEMATOPOIETIC GROWTH FACTORS & EPOGEN \\
\hline HEMATOPOIETIC GROWTH FACTORS & FILGRASTIM \\
\hline HEMATOPOIETIC GROWTH FACTORS & FILGRASTIM-SNDZ \\
\hline HEMATOPOIETIC GROWTH FACTORS & MIRCERA \\
\hline
\end{tabular}


HEMATOPOIETIC GROWTH FACTORS HEMATOPOIETIC GROWTH FACTORS HEMATOPOIETIC GROWTH FACTORS HEMATOPOIETIC GROWTH FACTORS HEMATOPOIETIC GROWTH FACTORS HEMATOPOIETIC GROWTH FACTORS IMMUNOSUPPRESSIVE AGENTS IMMUNOSUPPRESSIVE AGENTS IMMUNOSUPPRESSIVE AGENTS IMMUNOSUPPRESSIVE AGENTS IMMUNOSUPPRESSIVE AGENTS IMMUNOSUPPRESSIVE AGENTS IMMUNOSUPPRESSIVE AGENTS IMMUNOSUPPRESSIVE AGENTS IMMUNOSUPPRESSIVE AGENTS IMMUNOSUPPRESSIVE AGENTS IMMUNOSUPPRESSIVE AGENTS IMMUNOSUPPRESSIVE AGENTS IMMUNOSUPPRESSIVE AGENTS IMMUNOSUPPRESSIVE AGENTS IMMUNOSUPPRESSIVE AGENTS IMMUNOSUPPRESSIVE AGENTS METABOLIC MODIFIERS METABOLIC MODIFIERS METABOLIC MODIFIERS METABOLIC MODIFIERS METABOLIC MODIFIERS METABOLIC MODIFIERS METABOLIC MODIFIERS METABOLIC MODIFIERS OSMOTIC DIURETICS PHOSPHATE BINDER AGENTS PHOSPHATE BINDER AGENTS PHOSPHATE BINDER AGENTS PHOSPHATE BINDER AGENTS PHOSPHATE BINDER AGENTS PHOSPHATE BINDER AGENTS PHOSPHATE BINDER AGENTS PHOSPHATE BINDER AGENTS PHOSPHATE BINDER AGENTS PHOSPHATE BINDER AGENTS PHOSPHATE BINDER AGENTS POTASSIUM REMOVING RESINS POTASSIUM REMOVING RESINS POTASSIUM REMOVING RESINS POTASSIUM REMOVING RESINS POTASSIUM REMOVING RESINS POTASSIUM REMOVING RESINS SELECTIVE SEROTONIN REUPTAKE INHIBITORS (SSRIS) SELECTIVE SEROTONIN REUPTAKE INHIBITORS (SSRIS) SELECTIVE SEROTONIN REUPTAKE INHIBITORS (SSRIS)

\author{
NEULASTA \\ NEUPOGEN \\ PEGFILGRASTIM \\ PROCRIT \\ ROMIPLOSTIM \\ ZARXIO \\ ANTI-THYMOCYTE \\ AZATHIOPRINE \\ BASILIXIMAB \\ BELATACEPT \\ CELLCEPT \\ CYCLOSPORINE \\ EVEROLIMUS \\ IDS \\ MYCOPHENOLATE \\ MYCOPHENOLIC \\ MYFORTIC \\ NEORAL \\ PROGRAF \\ RAPAMUNE \\ SIROLIMUS \\ TACROLIMUS \\ CALCITRIOL \\ CINACALCET \\ DOXERCALCIFEROL \\ HECTOROL \\ PARICALCITOL \\ ROCALTROL \\ SENSIPAR \\ ZEMPLAR \\ MANNITOL \\ AURYXIA \\ CALCIUM \\ FERRIC \\ FOSRENOL \\ LANTHANUM \\ PHOSLO \\ RENAGEL \\ RENVELA \\ SEVELAMER \\ SUCROFERRIC \\ VELPHORO \\ KALEXATE \\ KAYEXALATE \\ KIONEX \\ PATIROMER \\ SODIUM \\ VELTASSA \\ CITALOPRAM \\ ESCITALOPRAM \\ FLUOXETINE
}


medRxiv preprint doi: https://doi.org/10.1101/2022.02.07.22270047; this version posted February 9, 2022. The copyright holder for this preprint (which was not certified by peer review) is the author/funder, who has granted medRxiv a license to display the preprint in perpetuity.

It is made available under a CC-BY-NC-ND 4.0 International license.

SELECTIVE SEROTONIN REUPTAKE INHIBITORS (SSRIS)

SELECTIVE SEROTONIN REUPTAKE INHIBITORS (SSRIS)

SELECTIVE SEROTONIN REUPTAKE INHIBITORS (SSRIS)

SELECTIVE SEROTONIN REUPTAKE INHIBITORS (SSRIS)

SELECTIVE SEROTONIN REUPTAKE INHIBITORS (SSRIS)

SPECIALTY VITAMINS PRODUCTS

SPECIALTY VITAMINS PRODUCTS

SPECIALTY VITAMINS PRODUCTS

SULFONYLUREAS

SULFONYLUREAS

SULFONYLUREAS

THROMBOLYTIC ENZYMES

VASODILATORS

VASODILATORS

VASODILATORS

FLUVOXAMINE

LEXAPRO

PAROXETINE

SERTRALINE

ZOLOFT

MG-PLUS

ONE-A-DAY

PROSTATE

GLIMEPIRIDE

GLIPIZIDE

GLYBURIDE

ALTEPLASE

HYDRALAZINE

MINOXIDIL

NITROPRUSSIDE 

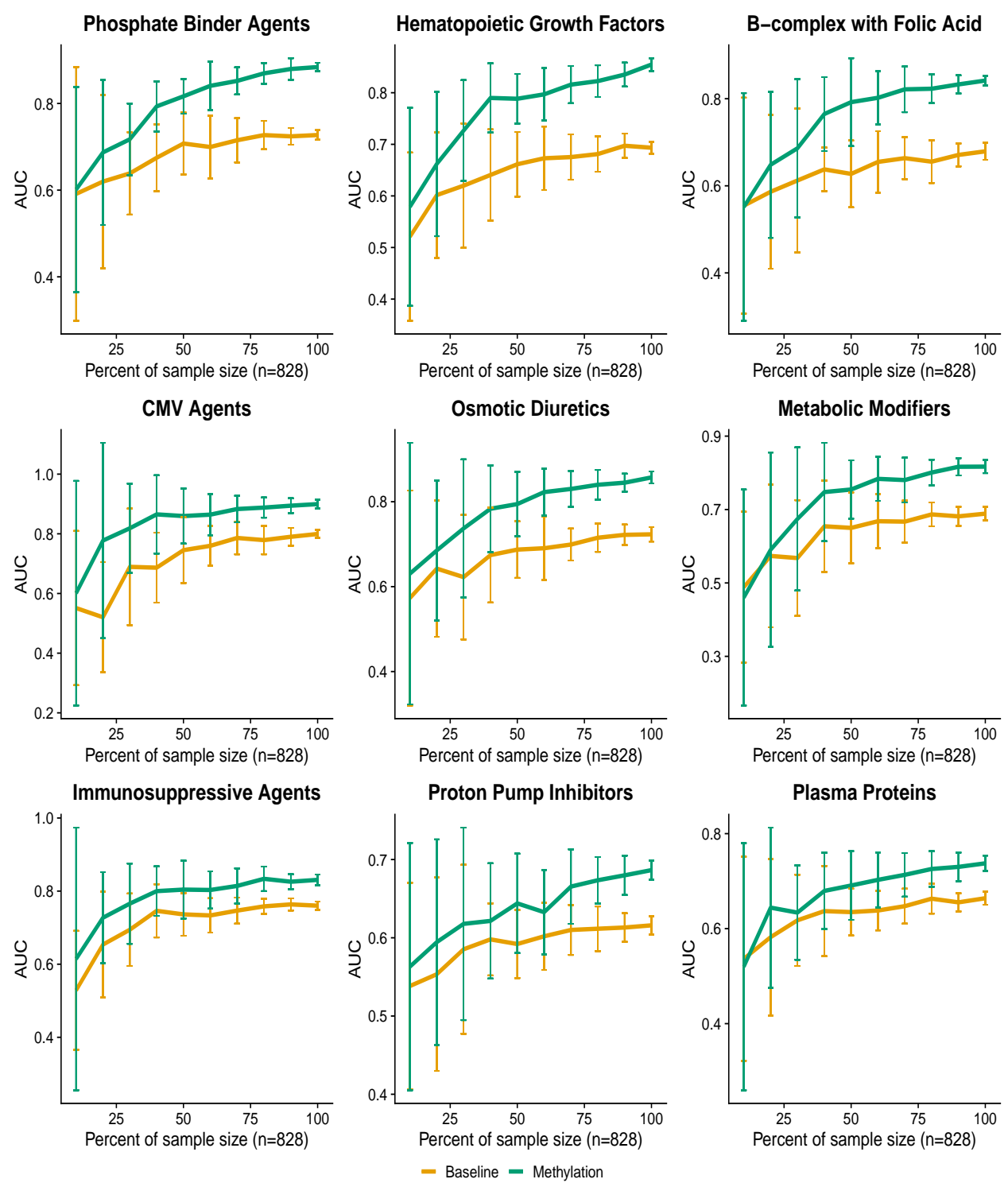

Figure S6. Downsampled performance on additional medications. We extended the downsampling experiments to include the top 10 most accurately imputed medications that also offered significant predictive power over the baseline features. We include here the remaining 9 medications. 

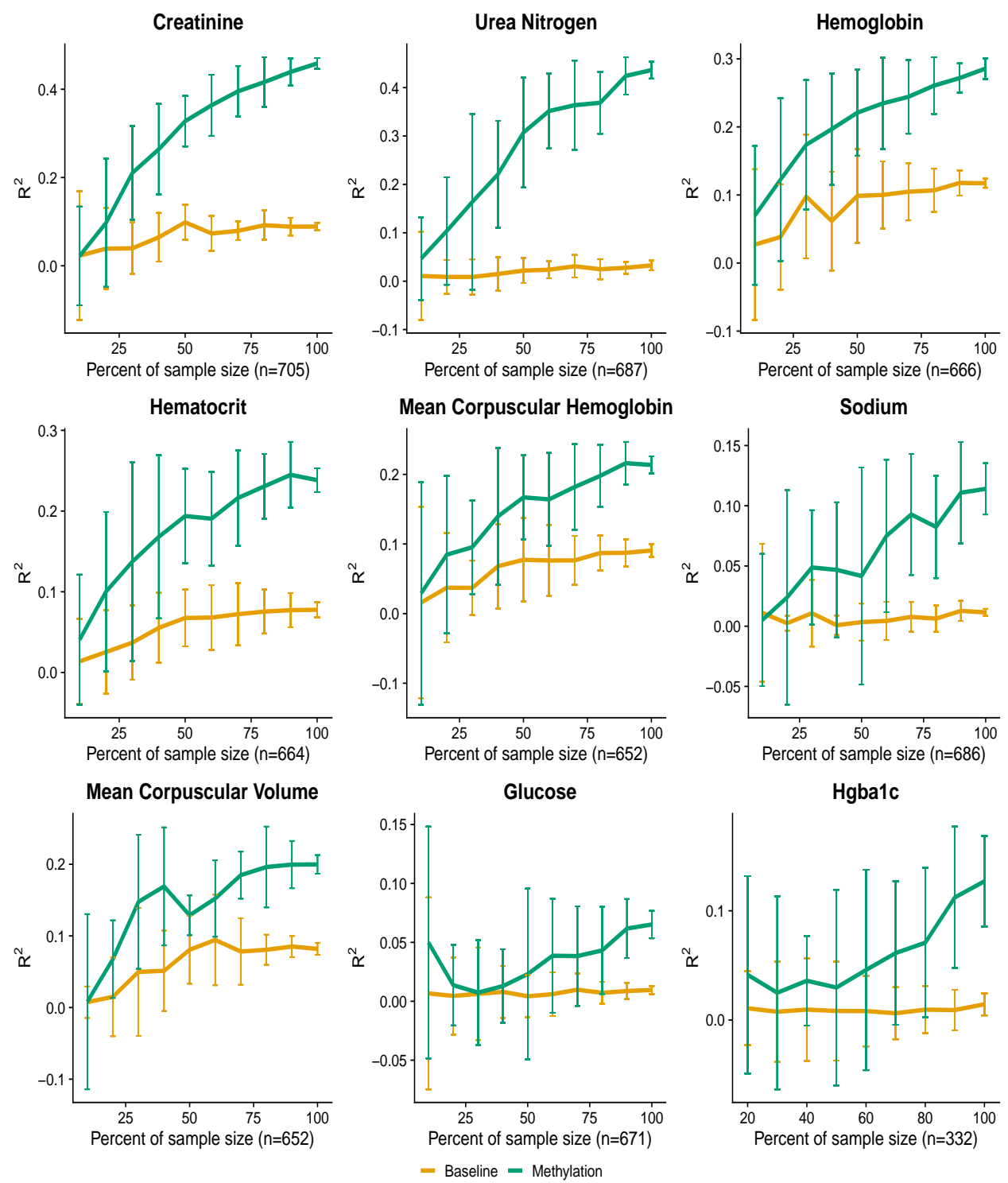

Figure S7. Downsampled performance on additional labs. We extended the downsampling experiments to include the top 10 most accurately imputed labs that also offered significant predictive power over the baseline features. We include here the remaining 9 labs. 

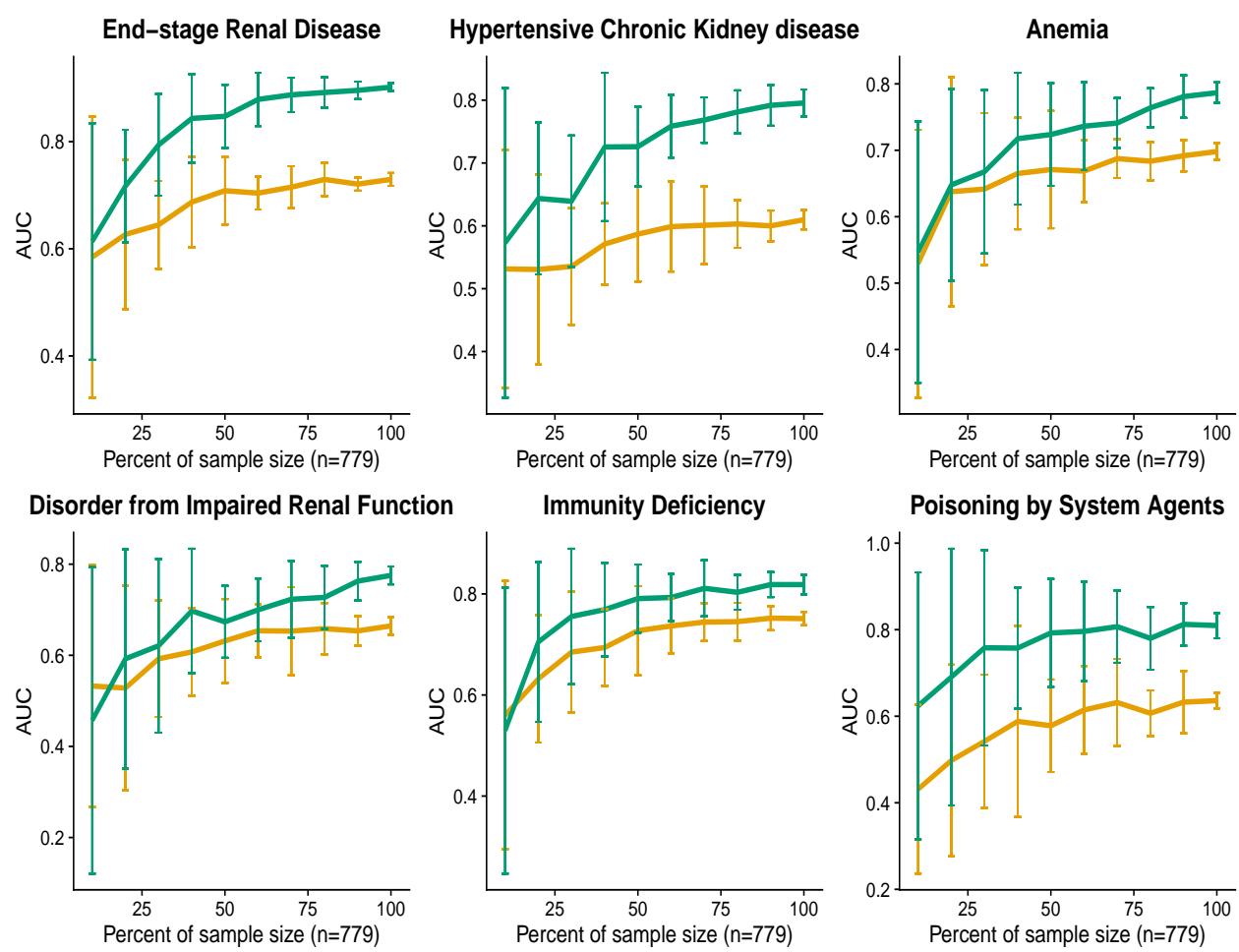

Poisoning by System Agents
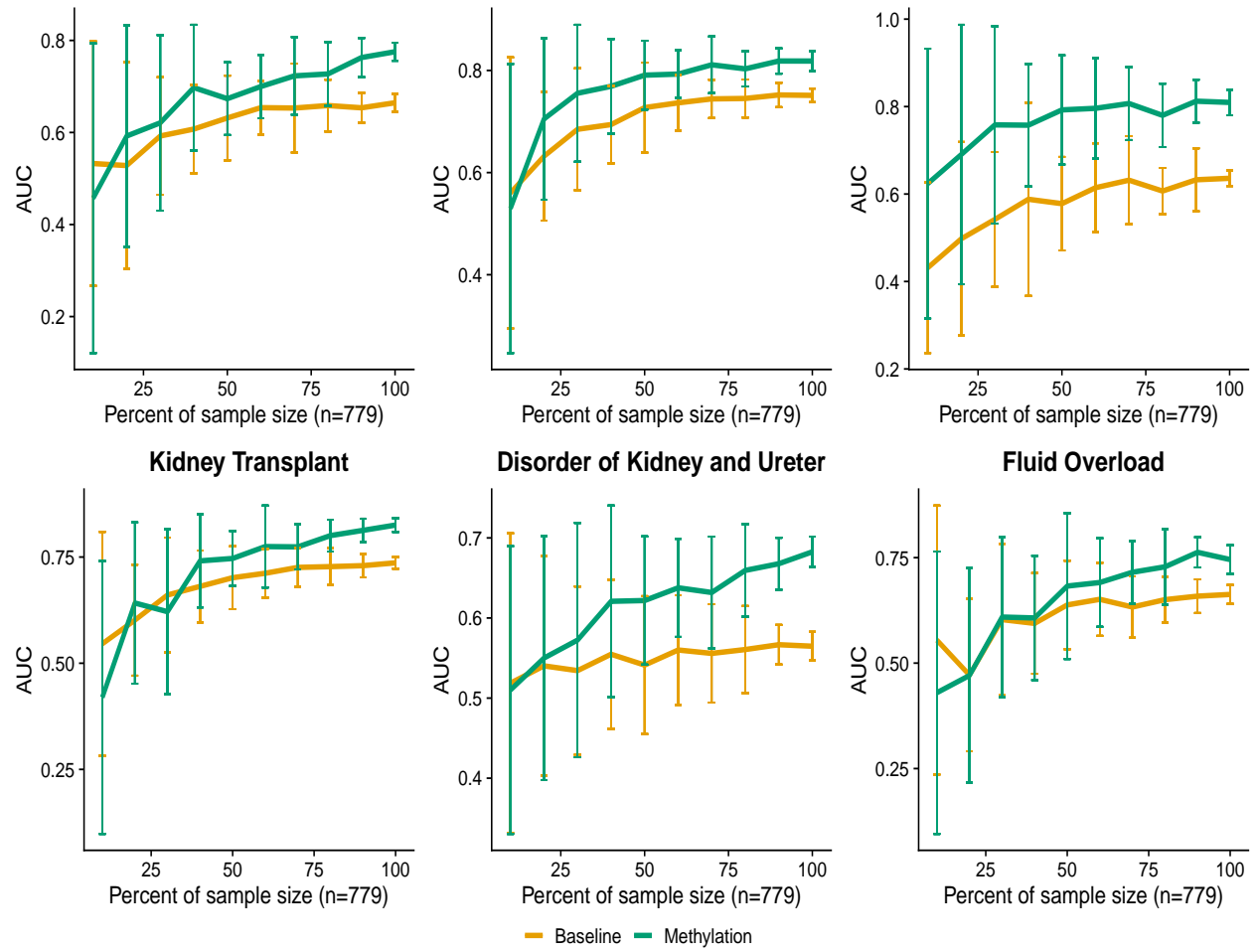

Figure S8. Downsampled performance on additional Phecodes. We extended the downsampling experiments to include the top 10 most accurately imputed Phecodes that also offered significant predictive power over the baseline features. We include here the remaining 9 Phecodes. 


\begin{tabular}{|l|c|c|c|c|}
\hline Lab & $\begin{array}{c}\text { PGS } \\
\text { accession }\end{array}$ & Study & $\begin{array}{c}\text { Number of } \\
\text { variants in } \\
\text { weight }\end{array}$ & $\begin{array}{c}\text { Number of } \\
\text { variants } \\
\text { present in } \\
\text { our data }\end{array}$ \\
\hline Albumin & & & & 9,172 \\
Cholesterol & PGS000669 & Sinnott-Armstrong et al. & 11,912 & 13,401 \\
Creatinine & PGS000677 & Sinnott-Armstrong et al. & 17,204 & 4,242 \\
HGBA1C & PGS000679 & Sinnott-Armstrong et al. & 5,469 & 11,208 \\
HDL & PGS000685 & Sinnott-Armstrong et al. & 14,658 & 19,123 \\
Hematocrit & PGS000686 & Sinnott-Armstrong et al. & 25,070 & 11,898 \\
Hemoglobin & PGS001225 & Tanigawa et al. & 15,721 & 11,770 \\
Mean corpuscular hemoglobin & PGS001400 & Tanigawa et al. & 15,602 & 13,003 \\
Mean corpuscular volume & PGS001219 & Tanigawa et al. & 17,311 & 13,181 \\
Urea nitrogen & PGS001220 & Tanigawa et al. & 12,351 & 9,473 \\
\hline
\end{tabular}

Table S10. Polygenic scores used for the imputed genotypes. We list below the weights used for computing the polygenic risk scores. We downloaded the weights from the Polygenic Score Catalogue (PGS) 55] from two studies of the UKBiobank 51, 53.
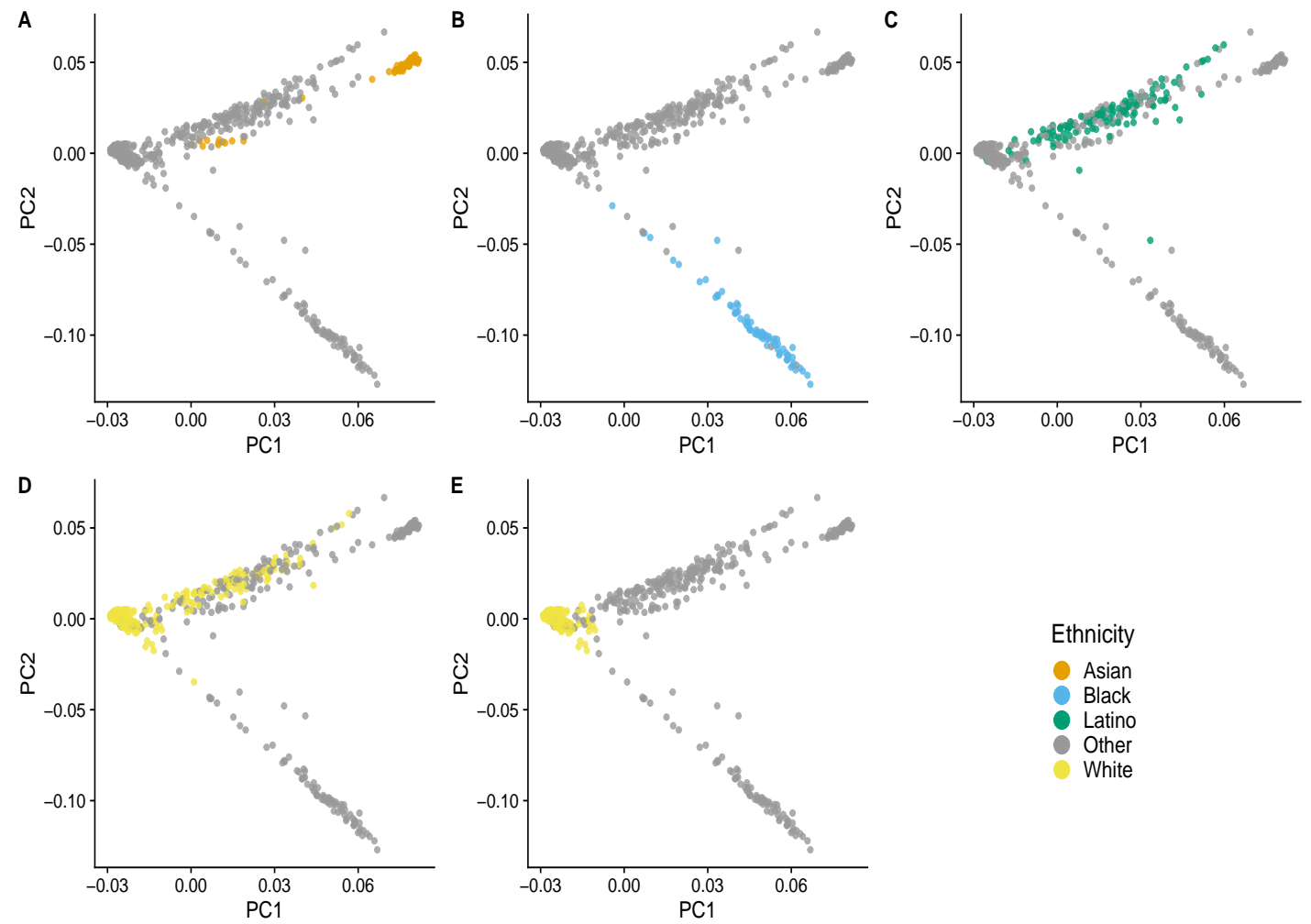

Figure S9. Self-reported ancestry along genetic PCs We show the primary self-identified ethnicity in each plot individually. For the analysis using external PRS we limited the set of white-identifying individuals to those who additionally had a $\mathrm{PC} 1$ score of $-<.01$. We show the individuals used in our analysis in plot E. 


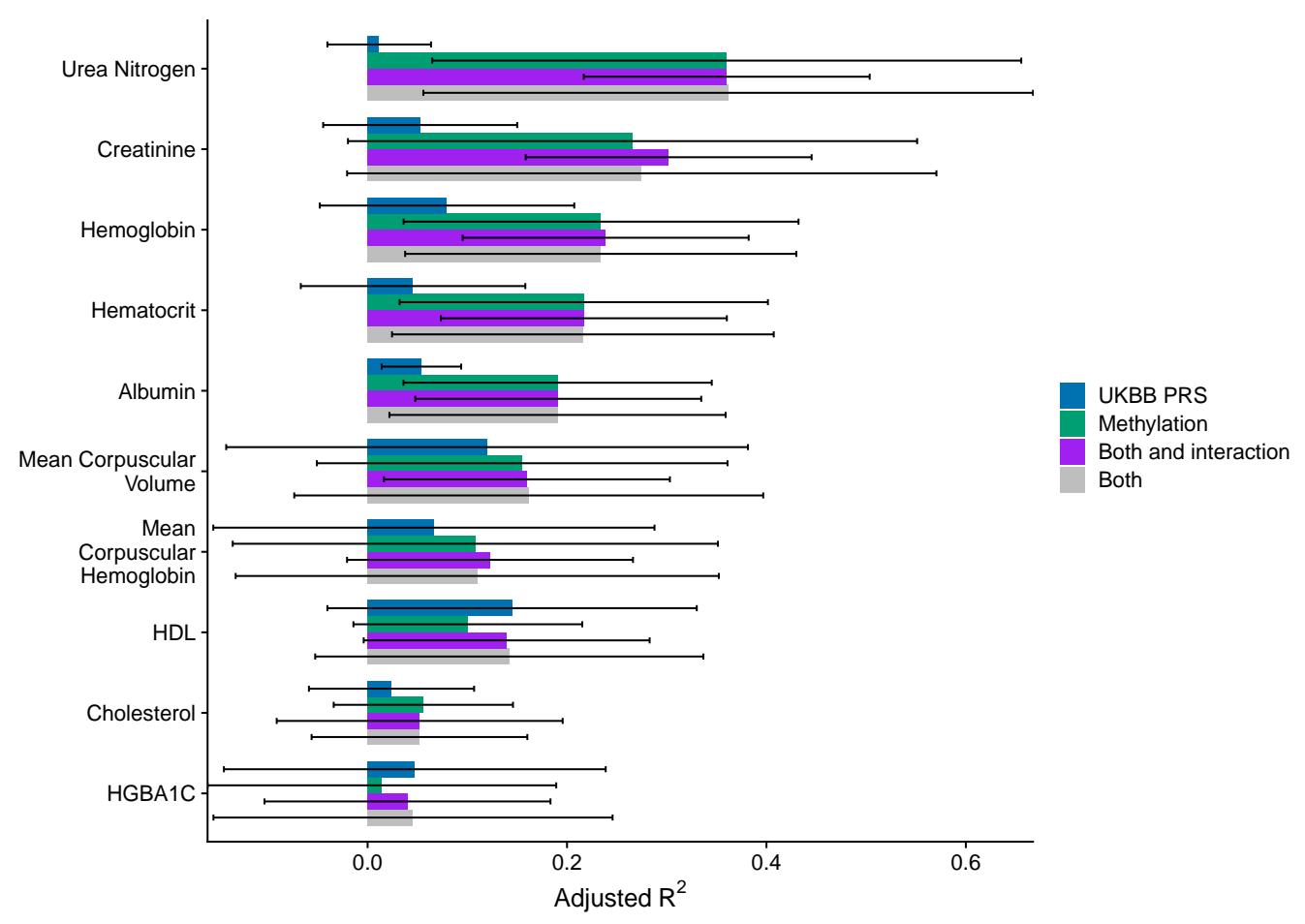

Figure S10. Labs as predicted by methylation, an externally-trained polygenic risk score, both, and a model that includes both as well as their interaction. The cross-validated adjusted $R^{2}$ between the true and imputed lab value on 541 unrelated patients of non-Hispanic-Latino whiteidentifying individuals using predictors that leveraged baseline features with either an MRS, a PRS externally-trained from the UKBiobank, both the MRS and the PRS, or a model that used both as well as the interaction between the MRS and PRS. Creatinine was the only outcome for which the interaction between both risk scores was statistically significant $(\mathrm{p}=9.16 \mathrm{e}-05)$, however the interaction for mean corpuscular hemoglobin was nominally significant ( $\mathrm{p}=1.44 \mathrm{e}-02)$. HDL corresponds to highdensity lipoprotein cholesterol and HGBA1C to glycated hemoglobin. 
medRxiv preprint doi: https://doi.org/10.1101/2022.02.07.22270047; this version posted February 9, 2022. The copyright holder for this preprint (which was not certified by peer review) is the author/funder, who has granted medRxiv a license to display the preprint in perpetuity.

\section{It is made available under a CC-BY-NC-ND 4.0 International license.}

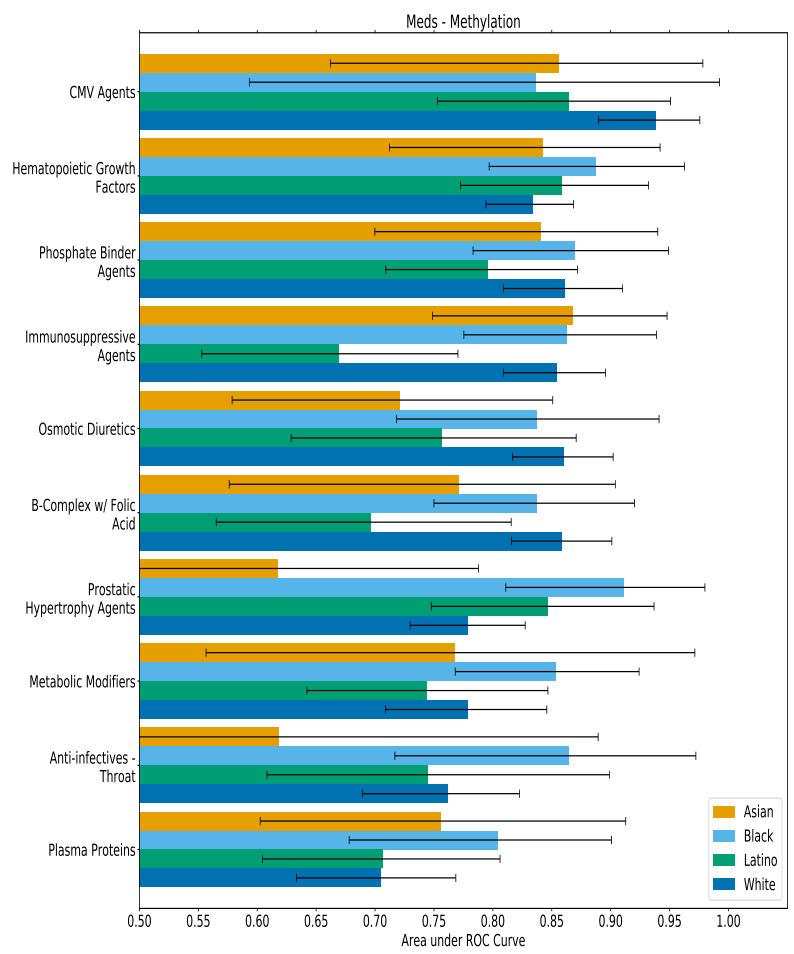

Figure S11. Best methylation-predicted medications within ancestral populations. After training a model on the entire heterogeneous set of individuals, we evaluated the predictive performance within each population separately. We observed no significant differences within self-reported ancestral groupings. 
medRxiv preprint doi: https://doi.org/10.1101/2022.02.07.22270047; this version posted February 9, 2022. The copyright holder for this preprint (which was not certified by peer review) is the author/funder, who has granted medRxiv a license to display the preprint in perpetuity.

\section{It is made available under a CC-BY-NC-ND 4.0 International license.}

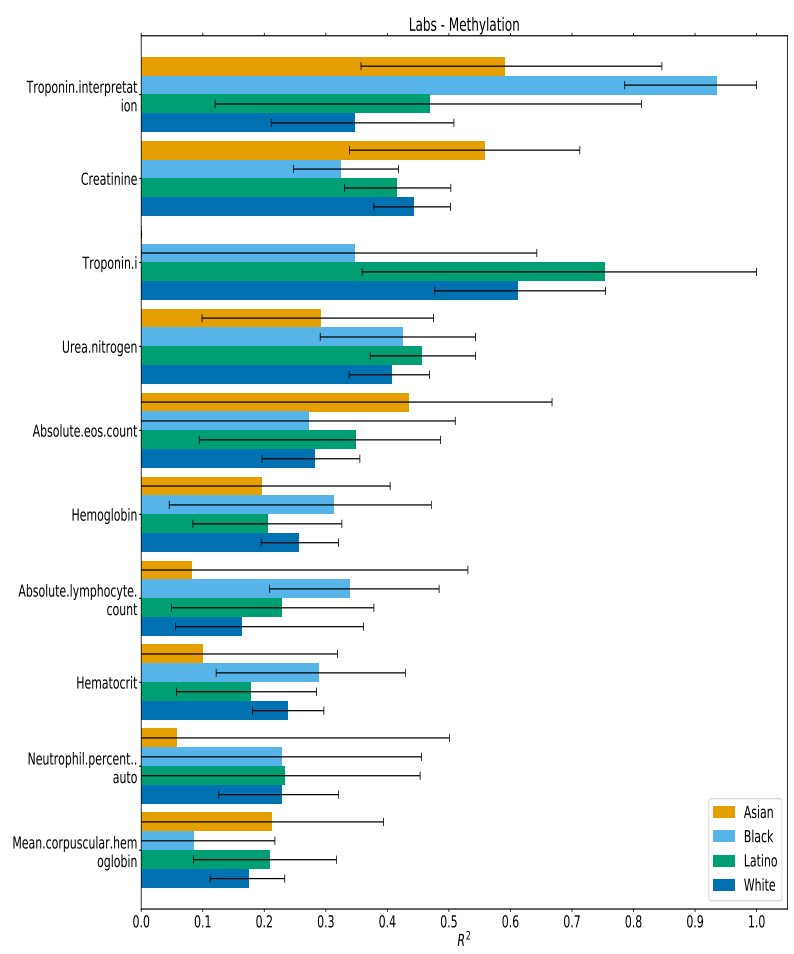

Figure S12. Best methylation-predicted lab panels within ancestral populations. After training a model on the entire heterogeneous set of individuals, we evaluated the predictive performance within each population separately. We observed no significant differences within self-reported ancestral groupings. 

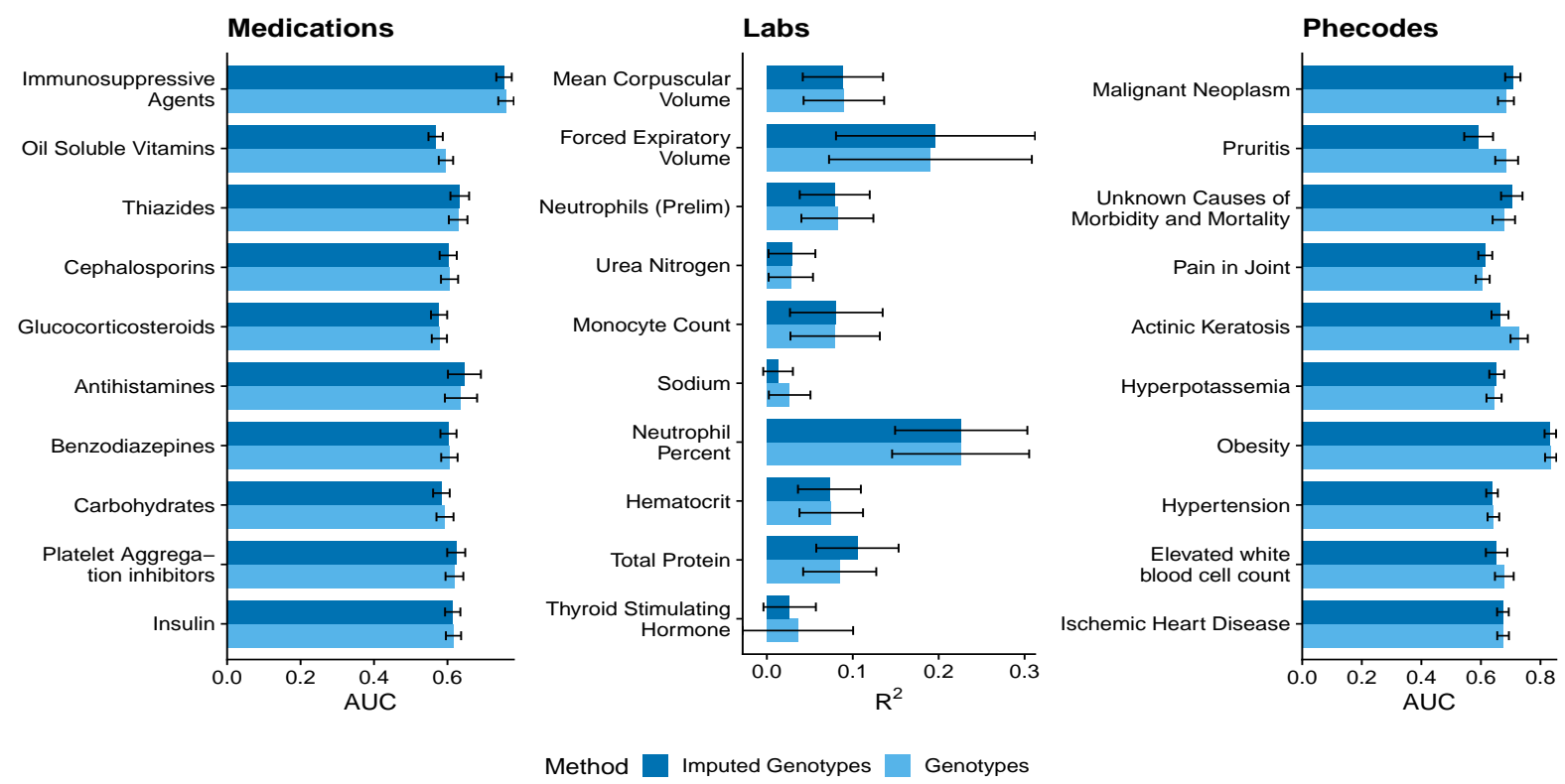

Figure S13. Imputation accuracy when constructing PRS using chipped genotypes compared to using imputed genotypes We fit models using the imputed genotypes on the outcomes that were best imputed by the chipped genotypes and that significantly improved over the baseline model. Using the imputed genotypes did not result in significant differences in imputation accuracy when compared to the chipped genotypes.

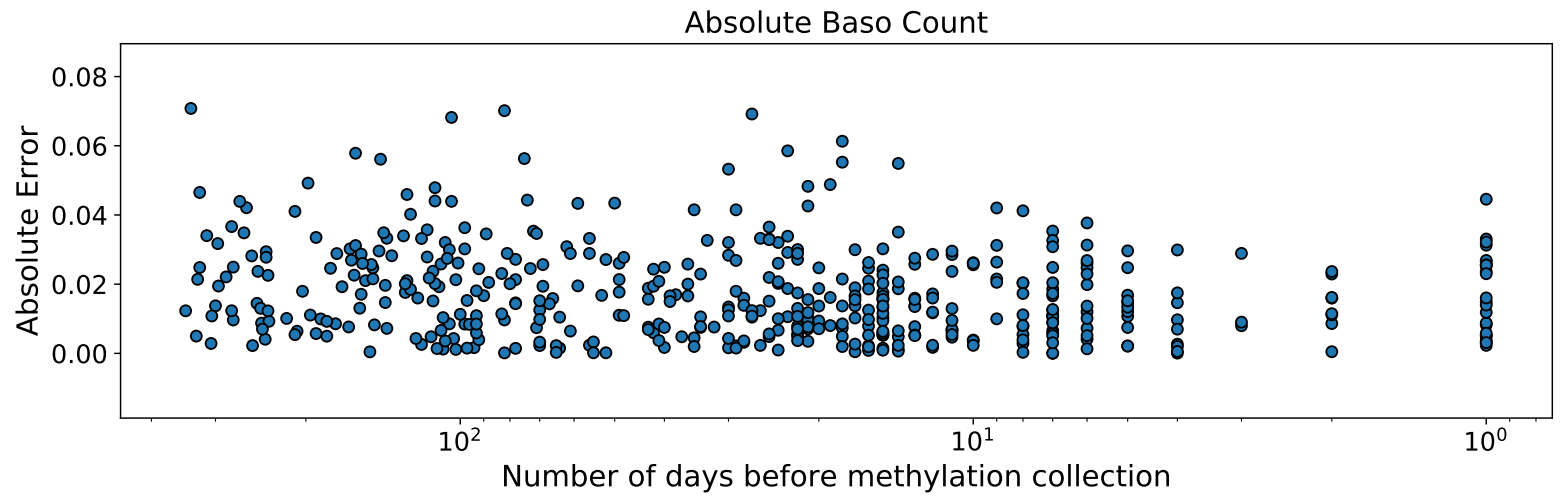

Figure S14. Imputation error as a function of time since methylation sample collection date We analyzed the lab value imputation residuals to see if the errors were associated with the number of days between the lab result and the methylation collection date. After correcting for multiple hypotheses, only one lab showed a significant association between the imputed value residuals and time between collection dates (absolute basophil count, Pearson $R=0.178$, Bonferroni corrected p-value $=0.0045$ ). Here we show the absolute residual error as a function of the number of days the lab resulted before methylation collection for the only significantly associated lab, absolute basophil count (note the log scaling on the $\mathrm{x}$-axis) 